\title{
Dielectric and Conductor-Loss Characterization and Measurements on Electronic Packaging Materials
}

James Baker-Jarvis Michael D. Janezic Bill Riddle Christopher L. Holloway

N.G. Paulter

J.E. Blendell
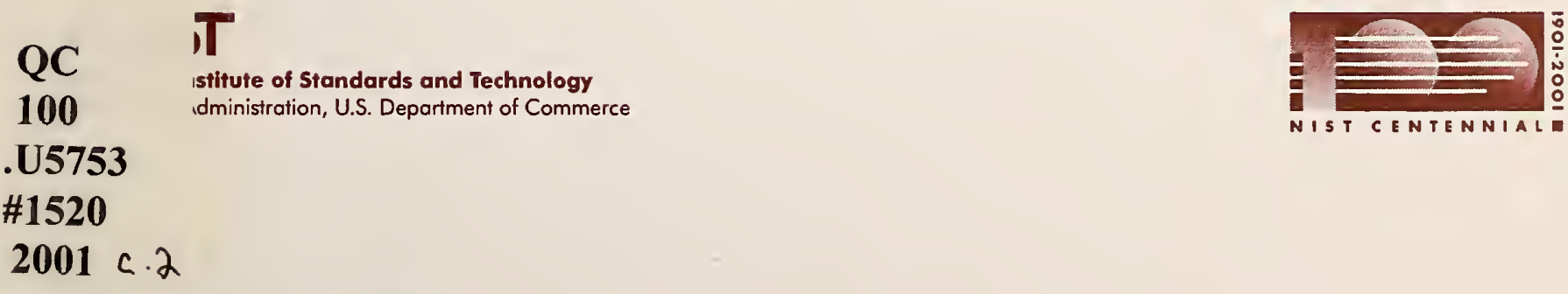



\title{
Dielectric and Conductor-Loss Characterization and Measurements on Electronic Packaging Materials
}

\author{
James Baker-Jarvis \\ Michael D. Janezic \\ Bill Riddle \\ Christopher L. Holloway \\ Radio-Frequency Technology Division \\ Electronics and Electrical Engineering Laboratory \\ N.G. Paulter \\ Electricity Division \\ Electronics and Electrical Engineering Laboratory \\ J.E. Blendell \\ Ceramics Division \\ Materials Science and Engineering Laboratory
}

July 2001

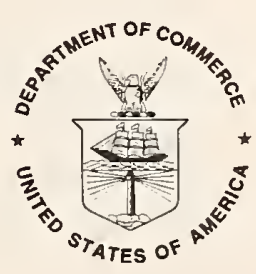

U.S. Department of Commerce Donald L. Evans, Secretary Technology Administration

Karen H. Brown, Acting Director National Institute of Standards and Technology 
National Institute of Standards and Technology Technical Note 1520

Natl. Inst. Stand. Technol. Tech. Note 1520, 156 pages (July 2001)

CODEN: NTNOEF 


\section{Contents}

1 Introduction $\quad 2$

2 Dielectric Materials 3

2.1 Overview of Packaging Technology and Materials . . . . . . . . . . . 3

2.2 Ceramic Substrates ................... 5

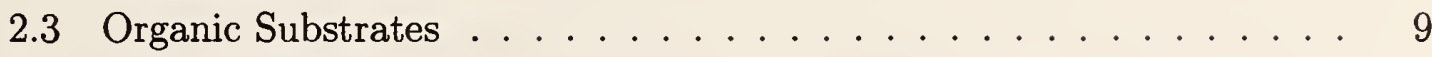

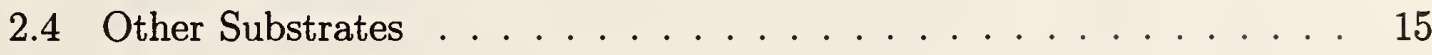

3 Dielectric Measurement Methods for the Permittivity and Permeability of Unmetalized Thin Substrates 17

3.1 Overview of the Measurement Problem . . . . . . . . . . 17

3.2 Resonant Methods ................... 27

3.2.1 Reentrant Cavity .................... 28

3.2.2 Split-Cylinder Resonators . . . . . . . . . . . 30

3.2.3 Cavity Resonators . . . . . . . . . . . . . . 30

3.2.4 $\mathrm{TE}_{01}$ Split-Post Dielectric and Magnetic Resonators . . . . . 31

3.2.5 Courtney Technique ............... 34

3.2.6 Whispering-Gallery Resonators ............ 36

3.2.7 Fabry-Perot Resonators . . . . . . . . . . . . 37

3.3 Transmission-Line Methods . . . . . . . . . . . . . . . 39

3.3.1 Coaxial Lines and Waveguides: Transmission/Reflection and Short-Circuited Line . . . . . . . . . . . . . . . . 39

3.3.2 Open-Ended coaxial lines and waveguides . . . . . . . 46

3.4 Capacitive Techniques ..................... 50

4 Dielectric Measurement and Metallic Loss

Characterization Using Patterned Fixtures $\quad 52$

4.1 Introduction to Metalized Structures . . . . . . . . . . . . 52 
4.2 Conductors on Printed Wiring Boards . . . . . . . . . . 53

4.3 Transmission-Line Parameters for

Circuit-Board Applications . . . . . . . . . . . . . . . . 55

4.4 Conductor-Loss Measurement . . . . . . . . . . . . . . . . . . 58

4.5 Time-Domain PWB Measurement Methods . . . . . . . . . . . . . 59

4.6 Full-Sheet Resonance Method for Thin Sheets . . . . . . . . . . . . 61

4.7 Techniques for Transmission Lines on Substrates . . . . . . . . . . . 64

4.7.1 Microstrip Resonator . . . . . . . . . . . . . . . . . 65

4.7 .2 Stripline . . . . . . . . . . . . . . . 70

4.7 .3 Coplanar Strip (CPS) $\ldots \ldots \ldots \ldots \ldots$

4.7.4 Coplanar Waveguide (CPW) . . . . . . . . . . . . . . . 80

4.7.5 Increased Power Loss Associated with Surface Roughness . . . 81

$4.8 \mathrm{~T}$ and Ring-Resonator Methods for Substrates . . . . . . . . . . 90

4.9 On-Wafer Transmission-line Methods for Substrates . . . . . . . . . . 90

5 Dielectric Measurement Software $\quad 92$

6 Ambient Temperature Measurements 93

7 Elevated Temperature Measurements 118

7.1 Thermal Properties . . . . . . . . . . . . . . . . . . . 118

7.2 Elevated Temperature Measurement Results . . . . . . . . . . . 122

8 Conclusion 131

9 References 132

$\begin{array}{ll}\text { Appendix A: Mixture Equations } & 151\end{array}$

Appendix B: Broadband-Plastic Measurements 152 


\title{
Dielectric and Conductor-Loss Characterization and Measurements on Electronic Packaging Materials
}

\author{
James Baker-Jarvis* Michael D. Janezic * \\ Bill Riddle* \\ Christopher L. Holloway * $\quad$ N. G. Paulter ${ }^{\dagger} \quad$ J. E. Blendell ${ }^{\ddagger}$
}

National Institute of Standards and Technology

325 Broadway, Boulder CO 80305

This is an overview of dielectric measurement methods and metrology on substrate materials used in electronic packaging. These substrates include low and high temperature co-fired ceramic substrates, printed wiring boards, and other packaging materials. The most commonly used methods are presented. We particularly emphasize methods that can be used nondestructively either in the laboratory or on the assembly line. We begin with an overview of pertinent electrical properties of substrate materials and conductors. We then describe frequency-dependent metal losses on conductors. We also overview cavity and dielectric resonators, full-sheet resonance, coaxial probe, stripline, and capacitive methods. The frequency ranges of applicability and typical uncertainties associated with the methods are summarized. We also present measurement results of many substrate materials.

Key words: dielectric; full-sheet resonance; loss factor; LTCC; metal loss; microwave; permeability; permittivity; printed wiring board; PWB; stripline; substrate.

\footnotetext{
*Radio-Frequency Technology Division, MS 813, email: jjarvis@boulder.nist.gov

$\dagger$ Electricity Division, MS 811, National Institute of Standards and Technology, Gaithersburg, MD 20899

${ }^{\ddagger}$ Ceramics Division, MS 852, National Institute of Standards and Technology, Gaithersburg, MD 20899
} 


\section{Introduction}

This technical note is an introductory overview of dielectric and magnetic measurement metrology on packaging materials.

Dielectric materials have many important functions in the microelectronics industry. New packaging technology requires substrates with low permittivity, interconnections made of high-conductivity metals, high wiring density, and embedded passive circuit elements. The use of fine-line signal conductors requires thinner, laminated, printed wiring board (PWB), thin films, low-temperature co-fired ceramics (LTCC), and other substrate materials. As electrical components are miniaturized, the need for well-characterized dielectric measurements on thin materials increases [1-10]. Accurate measurement of complex permittivity is needed for circuit design, minimization of crosstalk, and characterization of signal-propagation speed.

In Section 2 we review important fundamental concepts related to dielectric properties of microelectronic substrate materials. This includes the types of materials and laminates commonly used and the definitions of important electrical concepts. We also provide an review of the relevant parameters of transmission-line theory. In Section 3 we review various frequency-dependent permittivity measurement methods for thin materials. We also study metal losses in various transmission-line structures. In Section 6, we present measurements on substrate and circuit-board materials. In Section 7, we overview thermal properties and present a technique for measurements at elevated temperatures. Measurement results for a number of commonly used materials are also given. 


\section{Dielectric Materials}

\subsection{Overview of Packaging Technology and Materials}

Substrates are used in PWBs, central processing units, and for thin films. Important properties of LTCC, PWB, and other substrate materials include low electrical loss, high thermal conductance, low thermal expansion, and high interfacial adhesion to metal surfaces or other films (see table 1). Low electrical loss decreases heating and signal attenuation, high thermal conductivity rapidly removes heat from the circuit, and low thermal expansion promotes circuit durability.

In high-speed or high-frequency circuits, the speed of signal propagation is important. The signal-propagation delay depends on both the dielectric constant and the transmission-line structure. This dependence is manifest in the equation for propagation delay for transverse electromagnetic (TEM) propagation modes which, in a lossless line, is

$$
t_{d}=\frac{l \sqrt{\epsilon_{r}^{\prime} \mu_{r}^{\prime}}}{c}
$$

where $c$ is speed of light in vacuum, $l$ is line length, $\mu_{r}^{\prime}$ is the real part of the relative permeability given by $\mu=\mu_{0}\left[\mu_{r}^{\prime}-j \mu_{r}^{\prime \prime}\right], \epsilon_{r}^{\prime}$ and $\epsilon_{r}^{\prime \prime}$ are the real and imaginary parts of the relative permittivity, $\epsilon=\left[\epsilon_{r}^{\prime}-j \epsilon_{r}^{\prime \prime}\right] \epsilon_{0}$, and $\epsilon_{0}$ and $\mu_{0}$ are the permittivity and permeability of vacuum. $\epsilon_{r}^{\prime}$ provides a measure of the relative phase change as a sinusoidal signal propagates through a material. $\epsilon_{r}^{\prime \prime}$ is related to attenuation of the signal and includes both dielectric and dc-conductivity losses. Generally, the loss in a material is expressed in terms of the loss tangent, $\tan \delta=\frac{\epsilon_{r}^{\prime \prime}}{\epsilon_{r}^{\prime}}$. Dielectrics with low loss provide reduced attenuation and heating in circuits. They also provide greater signal integrity. The propagation delay per unit length in a lossless line can be expressed in terms of the distributed capacitance, $C$, and inductance, $L$

$$
t_{d}=\sqrt{\epsilon_{r}^{\prime} L C_{v a c}}
$$

where $L$ and $C_{v a c}$ are the inductance and equivalent vacuum capacitance of the 
Table 1. Summary of substrate properties and applications.

\begin{tabular}{c|cc}
\hline Property & Advantages & Desired range \\
\hline Low permittivity & Minimize cross talk & $\epsilon_{r}^{\prime} \approx 1-4$ \\
& $\begin{array}{c}\text { Decreased propagation delay } \\
\text { High-medium permittivity }\end{array}$ & \\
& Smaller circuits & $\epsilon_{r}^{\prime} \approx 8-500$ \\
Low loss & Generate phase changes & \\
& Less heat generated & $\tan \delta<5 \times 10^{-4}$ \\
Low thermal expansion & Less signal attenuation. & \\
& Less circuit failure & $\alpha<10^{-5}\left({ }^{\circ} \mathrm{C}^{-1}\right)$ \\
& Less impedance & \\
High thermal conductance & temperature dependence & \\
& Cooler operation & $k>30\left(\mathrm{~W} / \mathrm{m}^{\circ} \mathrm{C}\right)$ \\
\hline
\end{tabular}

line [11]. The propagation delay can be reduced by using substrates with a low value of $\epsilon_{r}^{\prime}$, by using different transmission-line structures such as microstrip, stripline, coplanar waveguide, coplanar strips, or by varying the geometries of signal line widths and conductor spacings of the transmission-line structure. Crosstalk is also an important parameter in the design of high-speed or high-frequency circuits. Crosstalk can increase the noise and spurious content on signal lines, which will affect both analog and digital circuits. A low value for $\epsilon_{r}^{\prime}$ can decrease the signal crosstalk between conductors by decreasing the capacitive coupling. Materials with low dielectric constants include Teflon ${ }^{1}$ (polytetrafluoroethylene (PTFE)), cross-linked polystyrene

\footnotetext{
${ }^{1}$ Specific materials that are commonly used are mentioned for informational purposes only. This does not imply or constitute any endorsement by the National Institute of Standards and Technology.
} 
(CPS), and fused silica $\left(\mathrm{SiO}_{2}\right)$.

High-permittivity materials also have a niche in microelectronic applications. At low frequencies, high-permittivity materials are used to keep dimensions of circuits small. This can be understood by considering how the TEM mode wavelength in a material, $\lambda$, is related to the permittivity and frequency, $f$, by

$$
\lambda \approx \frac{c}{f \sqrt{\epsilon_{r}^{\prime} \mu_{r}^{\prime}}} .
$$

Compact antenna arrays require high-permittivity substrates for maintaining phase shifts between elements. In addition, large capacitances can be obtained with highpermittivity materials. High-permittivity materials include silicon carbide, polycrystalline alumina, and monocrystalline materials, such as sapphire, lanthanum aluminate, aluminum nitride, ferrimagnetic materials, titanates, and gallium arsenide [1].

\subsection{Ceramic Substrates}

Ceramic substrate designs include thick film, plated copper, photo-patterned thick film, high-temperature co-fired ceramics (HTCC), and LTCC. The advantages of ceramic materials over polymers for substrates are durability, low thermal expansion coefficient, and relatively high thermal conductivity. High permittivity manifests itself in slower propagation speed and larger crosstalk in circuits. The propagation speed varies roughly as the square root of $\epsilon_{r}^{\prime}$. The permittivity of ceramics is strongly influenced by the microstructure [12]. Ceramics with lower than theoretical density have a lower permittivity and higher loss due to interfacial charges on the pore surfaces. Grain size also influences permittivity $[13,14]$. LTCC are commonly used in applications where ruggedness is important. LTCC are also being used in circuitry for cable-free wireless interconnections between devices, (for example: Bluetooth Technology).

The materials used for ceramic substrates [15] include aluminum oxide (alumina), cordierite $\left(2 \mathrm{MgO}_{2} \mathrm{Al}_{2} \mathrm{O}_{3} 5 \mathrm{SiO}_{2}\right)$, aluminum nitride, silicon carbide, silicon nitride, 
beryllium oxide, and boron nitride. LTCC materials usually are formed from alumina, cordierite or $\mathrm{MgO}$ combined with $\mathrm{SiO}_{2}$ /glass, with additions of $\mathrm{Pb}_{2} \mathrm{O}_{5}$ and $\mathrm{Ba}_{2} \mathrm{O}_{3}$. Beryllium oxide, aluminum nitride, alumina, silicon carbide, and silicon nitride have a permittivity higher than thoses of most organic materials [16,17]. Silicon carbide is a semiconductor and is often combined with $\mathrm{BeO}$ to obtain a low-loss substrate. Beryllium oxide, silicon carbide, and aluminum nitride have high thermal conductivity and are useful when rapid heat transfer is required. These materials have advantages when higher power levels are used. A potential problem with beryllium oxide, however, is its toxicity in powder form. Aluminum nitride has the disadvantage of being difficult to metalize and is lossy below $10 \mathrm{GHz}$.

Multilayer ceramic substrates can be classified by sintering temperatures. HTCC are usually composed of glass, alumina, and aluminum nitride. HTCC green tapes are sintered at approximately $1500^{\circ} \mathrm{C}$. LTCC green tape has a lower volumetric ratio of ceramic to glass than HTCC, and the sintering temperature is approximately 900 ${ }^{\circ} \mathrm{C}$. An advantage of low-temperature sintering is that a wider range of metals can be incorporated into a circuit. In LTCC technology, it is possible to fire the ceramics with embedded passives and conductors. LTCC has the advantage of allowing direct attachment to gallium arsenide and silicon circuits with high conductivity conductors. The capability of integrated multilayer technology is shown in figure 1 .

LTCC and HTCC ceramic substrates are made by mixing powders of ceramics, glass, organic binders, and solvents that are tape cast into large sheets of material. These green (unfired) tapes can be cut, punched, patterned, and laminated to form substrates of a desired geometry, as shown in figure 2. Some of the organic components are evaporated from the green tape during drying and the rest are decomposed during firing. Residual carbon left after firing tends to increase the permittivity [18]. Firing in a more oxidizing atmosphere can decrease the amount of carbon, but the choice of atmosphere may be limited due to the propeties of the metals used for conductors. 
Table 2. Resistivities of Metals $[1,18]$.

\begin{tabular}{c|ccccccccc}
\hline Material & $\mathrm{Cu}$ & $\mathrm{Ag}$ & $\mathrm{Au}$ & $\mathrm{Mo}$ & $\mathrm{Pb}$ & $\mathrm{Al}$ & $\mathrm{Sn}$ & $\mathrm{Pd}$ & $\mathrm{Pt}$ \\
\hline Bulk resistivity $(\mu \Omega . \mathrm{cm})$ & 1.7 & 1.6 & 2.3 & 5.2 & 20.6 & 2.65 & 11 & 10.8 & 10.6 \\
Thick-film resistivity $(\mu \Omega . \mathrm{cm})$ & 4 & 5 & 5 & 12 & - & - & - & - & - \\
Maximum temperature $\left({ }^{\circ} \mathrm{C}\right)$ & 950 & 950 & 950 & 1500 & 327 & 660 & 230 & 1550 & 1768 \\
\hline
\end{tabular}

There are many conductor materials used in ceramic-based circuits (see table 2). In this technology the conductors are applied as a paste or ink on the green-state substrates. The type of metal used as a conductor for a specific application is related to its melting temperature, resistivity, migration resistance, cost, line resolution, and solderability. Typical conductors used in LTCC are aluminum, copper, gold, silver, and palladium- silver alloys. Gold produces a very high definition and reliable circuit, but is expensive. Silver tends to migrate into substrates causing warping of the substrate. HTCC requires metals of higher melting temperature, such as molybdenum, palladium, platinum, and tungsten. In the firing process, the conductor's properties change and carbon is generated in the substrate from decomposition of the organic binders, which increases both the effective permittivity and the loss.

Ceramic multichip modules (MCM-C) can be fabricated using LTCC materials. The green ceramic tapes can be patterned and machined, and then pressure laminated and fired. Integrated passive components such as inductors, resistors, and capacitors are easily incorporated into LTCC design. Cavities for filters can also be included. LTCC can have a large number of layers, for example 40 or more [19]. Interlevel connections are obtained through drilled or punched via holes and also through heat transfer vias. The embedded passives include resistors made from lossy metallic films yielding resistances from milliohms to megaohms, inductors made from spiraled or serpentine conductors of width $125 \mu \mathrm{m}$ to $150 \mu \mathrm{m}$, and capacitors made 
from high-permittivity materials yielding capacitances from $1 \mathrm{pF}$ to $1000 \mathrm{pF}$. The change in resistivity in co-fired resistors as a function of temperature is reviewed in reference [20].

The ability to include components into the modules reduces the number of interconnects which increases reliability and reduces the size and the cost. LTCC technology also allows for a high density of signal lines throughout the module and vias as small as $0.075 \mathrm{~mm}$ in diameter. Another advantage of MCM-C over PWBbased multichip modules is the lower thermal expansion coefficient. Heat transfer is facilitated by via arrays mounted on high-thermal conductivity materials such as AlSiC.

The permittivity of low-loss ceramics is relatively constant with frequency. In most ceramics the loss tangent increases as the frequency increases. The loss tangent for many ceramic materials and some polymers obey a quasi-linear dependence with frequency $f$, of the form $\tan \delta=\mathrm{a} f+\mathrm{b}$, where a is usually a positive number. This dependence is not applicable to all ceramics, for example, aluminum nitride.

Low-loss ceramic dielectric materials within a cavity can resonate if excited by an electric field of the appropriate frequency. Useful high- $Q$ resonators can be made by enclosing a dielectric in a metallic structure. Dielectric resonators are used as oscillators, filters for rejection of unwanted frequencies, and combiners. Examples of dielectric resonators used in the wireless industry are shown in figure 3 and are made of low-loss, temperature stable, high-permittivity materials. Materials commonly used for high-Q resonators include alumina, sapphire, and titanate compounds. The relevant properties and requirements for dielectric resonators are summarized in table 4.

Polymers have been combined with barium titanate powder to obtain a substrate of relatively high effective permittivity, that is less brittle than a pure ceramic board, but with a higher loss [21]. 
Table 3. Ceramic and glass substrates [1].

\begin{tabular}{c|cc}
\hline $\begin{array}{c}\text { Material } \\
\text { or composite }\end{array}$ & $\begin{array}{c}\text { Operational } \\
\text { temperature range }\left({ }^{\circ} \mathrm{C}\right)\end{array}$ & Anisotropy \\
\hline Alumina & -200 to 300 & no \\
Sapphire & -200 to 300 & yes \\
Fused silica & -200 to 500 & no \\
Rutile & -200 to 500 & yes \\
Beryllium oxide & -100 to 300 & no \\
Aluminum nitride & -200 to 300 & no \\
Borosilicate/Alumina & -200 to 500 & no \\
\hline
\end{tabular}

\subsection{Organic Substrates}

Organics (plastics or polymers) are commonly used in packaging materials. Substrate materials for PWB are usually composite organic materials and may be anisotropic. Laminations and woven-glass cloth are usually the cause of dielectric anisotropy. Examples of laminates are fiberglass-epoxy composites (FR-4), high-temperature fiberglass-epoxy composites (FR-5), bismalimide triazine-epoxy, cyanate ester, arimidepoxy, polyimide-glass, and polyimide-quartz [22]. The composites commonly consist of a mixture of plastics, glass, and/or ceramics, together with reinforcing materials. Plastics usually are reinforced with glass fibers or impregnated with glass or ceramics. Typical reinforcing materials used are paper fabric, woven glass cloth, random fiberglass fibers, and aramid fiber cloth (see figure 4). The fabric and fiber weaving have some variability due to manufacturing limitations, and this translates into a variability in permittivity. 


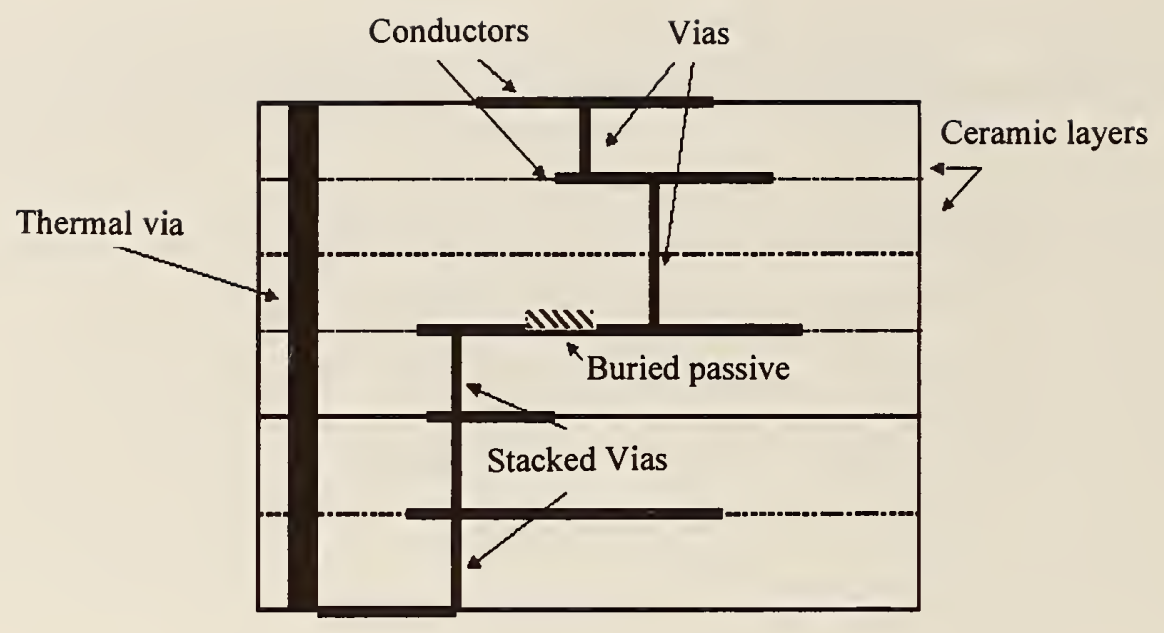

Figure 1. Geometry of typical LTCC substrate or multilayered PWB.
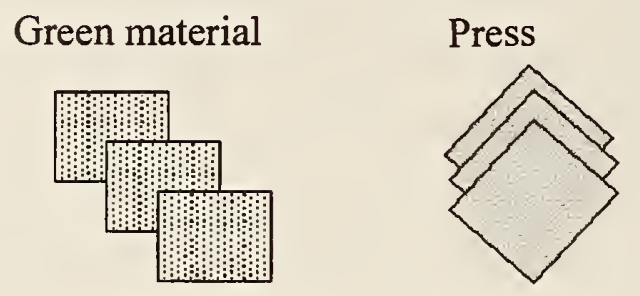

Fire

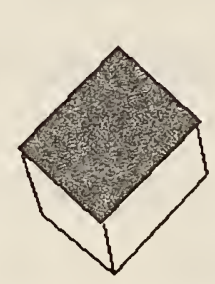

Figure 2. Fabrication steps of LTCC substrates.

There are two types of organic wiring boards, rigid PWB and flexible printed wiring board (FWB). Rigid PWBs are fabricated from fiber-reinforced thermoset polymers or ceramics. FWBs are formed from polymer films. Composite PWB boards are laminates of plastic or ceramic materials. Multichip PWB modules (MCM-L) are used to increase wiring density and are fabricated with laminated PWB boards. Interlevel connections are obtained through via holes.

Metal foils are electrodeposited or are attached to PWB laminates by adhesives composed of polymidies, epoxies, acrylics, and polyesters. A cross section of a single- 

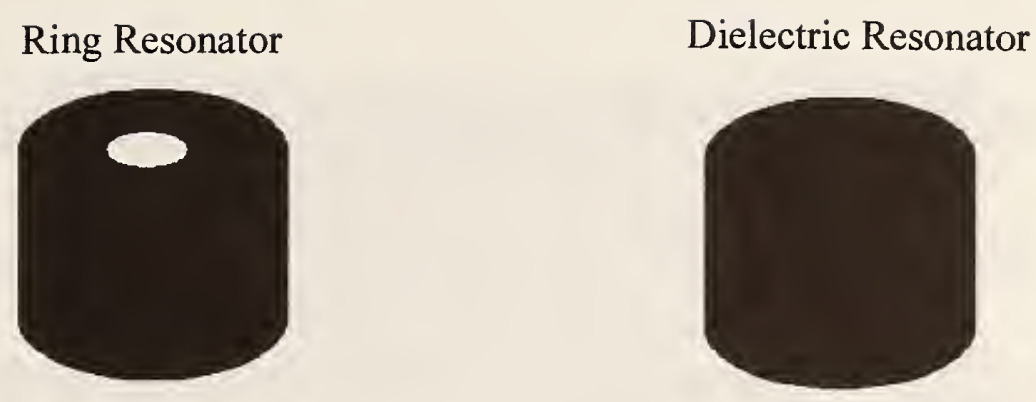

Figure 3. Two types of dielectric resonator.

Table 4. Summary of important properties for dielectric resonators.

\begin{tabular}{cc}
\hline Property & Requirements \\
\hline High permittivity & Miniaturization \\
Low loss & Large Q, close channel spacing \\
Low thermal expansion & Frequency stability \\
High thermal conductance & Cooler operation \\
\hline
\end{tabular}

layer PWB is shown in figure 5 and that for a two-layer system in figure 6 . The foils are from $20 \mu \mathrm{m}$ to $100 \mu \mathrm{m}$ thick. These copper foils are patterned, typically by etching, to form interconnect lines.

The properties of plastic materials are summarized in tables 5 and 6 . Typical bulk polymers are PTFE, CPS, fiberglass, polyimides, fluropolymers, and composites made from fiberglass, fused silica, ceramics, polyethylene, aerogels, and foams. These polymers all have low $\epsilon_{r}^{\prime}$. Plastics, however, have large coefficients of thermal expansion (CTE) and low mechanical strength. Moreover, the thermal expansion coefficients have a nonlinear temperature dependence. Very low $\epsilon_{r}^{\prime}$ can be achieved by introducing porosity, use of low-permittivity materials, or by forming hollow stripline 


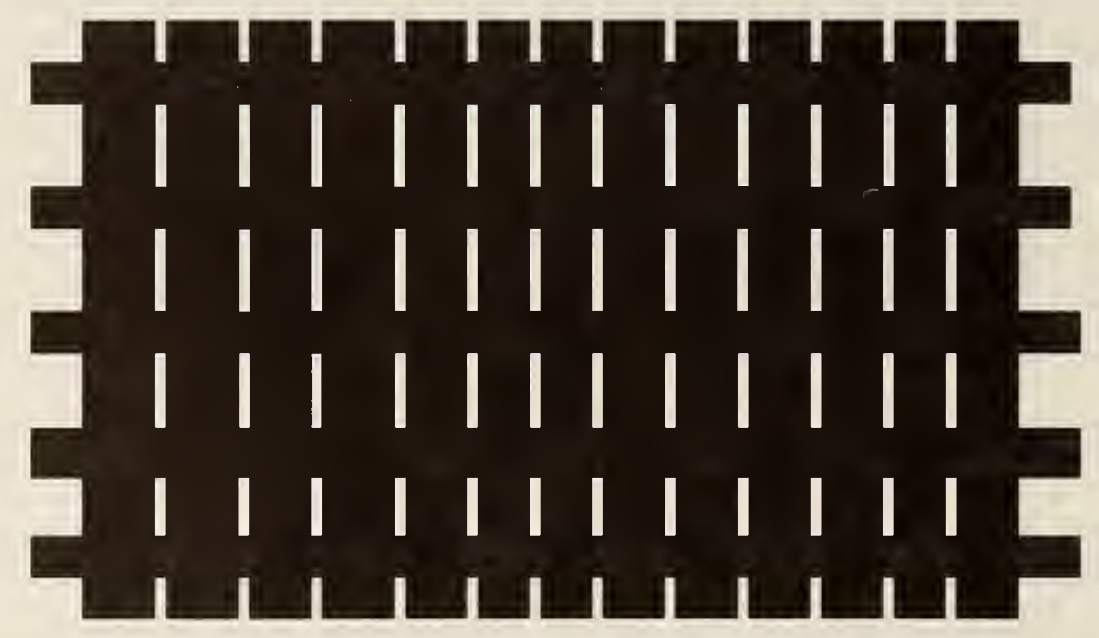

Figure 4. Weaving structure in glass FR-4.

ceramic structures [23].

Polymer resins are classified into thermoplastics or thermosets. Thermoplastics, for example polyethylene or polystyrene, soften when heated. Thermosets, such as epoxies and phenolics, do not soften or melt when heated. Epoxies are polyethers and have good chemical resistance and adhesive properties. Common resins for lowcost laminates are phenolics. Phenolics, such as Bakelite, are thermosetting plastics. When heat and pressure are applied in the moulding process, thermosetting plastics react to form a cross-linked structure. This structure yields excellent dimensional and thermal stability and superior load-bearing capability at low to near glass-transition temperatures. Recently, more complex low-permittivity polymers such as polyquiolines and benzocyclobutenes have been developed. Phenolic resins are products of the condensation reaction of phenol and formaldehyde. Other polymers used in PWB materials are polyimides, cyanate ester [24], bismalimide triazine 


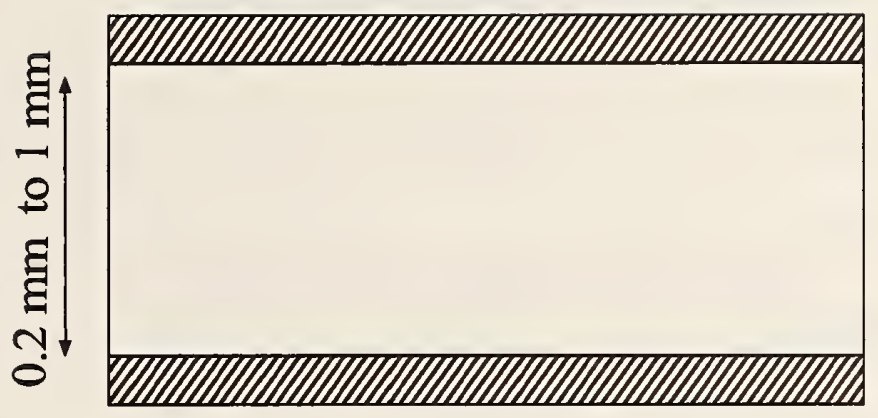

Figure 5. Geometry of PWB metal-clad substrates.

(BT), fluropolymers, and polysulfone. Polyimides, bismalimide triazine, and cyanate ester can withstand higher temperatures than can other polymers. Fluropolymers and polysulfone have low dielectric loss. Polymers may be crystalline, amorphous, or a combination. Dielectric properties of these materials are functions of both frequency and temperature. Dielectric properties of many commonly used polymer materials are summarized in [25].

Cost is crucial for the widespread acceptance of a specific material. In PWB applications, epoxy-glass, such as FR-4, is the least expensive material and, as a consequence, holds a large market share. There are many variations of FR-4 epoxyglass materials, with a variation in permittivity from 3.8 to 4.6 . In increasing order of cost are epoxy-glass, polyimide, polyimide-quartz, and PTFE.

The reinforcements in PWB include inorganic and organic glass fibers as shown in figure 4. For example, aramid is a fiber made from Kevlar. Teflon and fused silica fibers are also used. Laminates used in PWB include E-glass/epoxy (FR-4), glass/polyimide, aramid/polyimide, aramid/epoxy, glass/PTFE. The polymer films 
Table 5. Typical properties of plastic substrates [1].

\begin{tabular}{c|cccc}
\hline $\begin{array}{c}\text { Material } \\
\text { or composite }\end{array}$ & $\begin{array}{c}\epsilon_{r}^{\prime} \\
\text { (average) }\end{array}$ & $\tan \delta$ & $\begin{array}{c}\text { Useful range of } \\
\text { temperatures }\left({ }^{\circ} \mathrm{C}\right)\end{array}$ & Anisotropy \\
\hline PTFE & 2.06 & 0.0002 & -20 to 150 & no \\
PTFE/Fiberglass & 2.25 & 0.0010 & -20 to 150 & yes \\
PTFE/Quartz & 2.50 & 0.0005 & -20 to 200 & \\
PTFE/Ceramic & 10.0 & 0.0010 & -20 to 200 & \\
CPS & 2.54 & 0.0004 & -20 to 100 & no \\
CPS/Fiberglass & 2.70 & 0.0010 & -20 to 100 & yes \\
CPS/Quartz & 2.70 & 0.0005 & -20 to 100 & yes \\
Polyolefin & 2.30 & 0.0005 & -20 to 100 & no \\
Polyimide & 3.50 & 0.0010 & -20 to 130 & no \\
\hline
\end{tabular}

Table 6. General properties of substrate materials.

\begin{tabular}{c|cccc}
\hline Class & Permittivities & Range of tan $\delta$ & CTE & Metal Adhesion \\
\hline low-K polymers & $2-5$ & $0.0001-0.01$ & poor & poor-good \\
Plastic/Ceramic composites & $3-50$ & $0.0001-0.01$ & good & poor-good \\
Glasses & $3-12$ & $0.00005-0.01$ & good & good \\
Ceramics & $10-500$ & $0.00005-0.005$ & good & good \\
Ceramic/Glass composites & $4-100$ & $0.00005-0.005$ & good & good \\
\hline
\end{tabular}




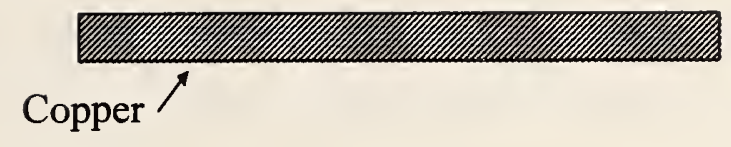

Material 1

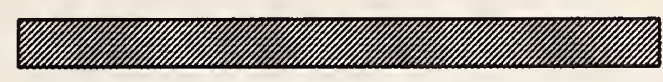

Material 2

Figure 6. Dual-material PWB structure.

in FWB are primarily PTFE, polyimides, and nylon.

\subsection{Other Substrates}

There are a number of other materials commonly used as substrates. In this section we overview some of the properties of other substrates [26]. Semiconducting materials such as silicon and gallium arsenide are used as substrates. Silicon is very lossy at low frequencies, whereas gallium arsenide has low loss. A substrate becoming more commonly used is gallium nitride. Anisotropic substrates are single-crystal sapphire, rutile, silicon, and quartz. Sapphire has a high thermal conductivity and very low dielectric loss. Sapphire and quartz are both brittle and difficult to drill and cut. Glasses such as fused silica and borosilicate have low loss and medium values of permittivities. Fused silica and most glasses have poor thermal conductivity. Rutile 
has a very high permittivity and medium loss.

Tunable or frequency-agile ferroelectric and ferrite materials in bulk or thin-film form are being used for device design and consequently there is a need for unbiased and biased dielectric and magnetic measurements. In ferromagnetics or ferrimagnetics, the permeability can be a function of the applied magnetic field. Permittivity tuning by applied voltage requires strong electric fields, so most ferroelectric materials are used as thin films. In ferroelectric materials the permittivity is a function of the applied bias field. Microscopic domains consisting of electric dipoles are formed from strong dipole-dipole interactions in ferroelectric materials. Ferroelectric material losses are affected strongly by temperature. The loss tangent of ferroelectrics increases with the temperature approximately as $T^{2}$. Applications for ferroelectric materials include phase shifters for antenna arrays. The loss in these materials is minimized by adding manganese to the solid. The most commonly used ferroelectric material is barium-strontium titanate. High-temperature performance of dielectric and ferromagnetic materials is also important for high-power systems. 


\section{Dielectric Measurement Methods for the Permittivity and Permeability of}

\section{Unmetalized Thin Substrates}

In this section we review a number of dielectric measurement methods for unmetalized or unpatterned materials. In Section 3.1 we overview the important issues surrounding dielectric measurements and in Section 3.2 unmetalized resonant dielectric measurement methods are overviewed. We then present a summary of waveguide and coaxial line measurement methods. Metalized-substrate dielectric measurement methods are overviewed in Section 4.

\subsection{Overview of the Measurement Problem}

Dielectric properties of a specimen depend on frequency, homogeneity, anisotropy, temperature, surface roughness and, in the case of ferroelectrics, applied dc bias field $[2,27]$. Dielectric materials may be isotropic or anisotropic, crystalline, amorphous, clad or unclad, bulk or film. No single technique can accurately characterize all materials over all frequencies and temperatures. Each frequency band and loss regime usually requires a different method.

Anisotropy in PWB materials is usually a result of weaving fiber in the material. In bianisotropic materials, there are two independent permittivity components. In such cases we distinguish between in-plane and out-of-plane permittivity. In table 7 , some of the various parameters pertaining to permittivity measurements are summarized. The measurement of thin materials presents a special challenge in that uncertainties in thickness of the specimen translates into uncertainty in the permit- 
Table 7. Measurement parameters.

Component of permittivity (in-plane or normal)

Frequency

Loss measurement

Specimen thickness

Temperature

tivity. In this report, we consider thin materials as those having a thickness less than $3 \mathrm{~mm}$. Measurement methods on thin films that depend less on sample thickness and more on transverse dimensions yield more accurate results for $\epsilon_{r}^{\prime}$.

The reentrant cavity, stripline, microstrip, and full-sheet resonance techniques are useful for measurement of the component of permittivity normal to the sample face. However, if the sample is not metalized, measurements with this type of field orientation can suffer from the effects of air gaps between the sample and electrode. $\mathrm{TM}_{0 m}$ cavities can yield a measurement with the electric field normal to the specimen face. Using higher modes, these cavities can measure over a frequency band of $1 \mathrm{GHz}$ to $10 \mathrm{GHz}$. The stripline technique has the advantage of easily accommodating PWB materials. It gives reasonable estimates for $\epsilon_{r}^{\prime}$, but dubious estimates for $\epsilon_{r}^{\prime \prime}$. The split-cylinder and split-post resonators are useful for obtaining in-plane permittivity and loss. Whispering-gallery modes can be used to obtain very accurate measurements on low-loss samples. Fabry-Perot resonators are useful at high frequencies. The coaxial probe techniques are useful in obtaining permittivity of isotropic materials with medium accuracy. Many of the important features of the most important measurement techniques are summarized in tables 8 and 9 .

The full characterization of thin, anisotropic materials generally requires two 
Table 8. Dielectric measurement categories compared with typical uncertainties with each method.

\begin{tabular}{c|cccc}
\hline Technique & Field & Advantages & $\Delta \epsilon_{r}^{\prime} / \epsilon_{r}^{\prime}$ & $\Delta \tan \delta_{r}$ \\
\hline Full-sheet reson. & $\mathrm{TE}_{10}$ & $\epsilon_{r}^{\prime}$ & $\pm 2 \%$ & \\
coaxial Line & $\mathrm{TEM} \mathrm{TE}_{10}$ & Broadband & $\pm 1-10 \%$ & \pm 0.005 \\
or waveguide & & & & \\
Capacitor & Normal E-field & Low f & $\pm 1 \%$ & $\pm 10^{-4}$ \\
Cavity & $\mathrm{TE}_{01}$ & Very Accurate & $\pm 0.2 \%$ & $\pm 5 \times 10^{-5}$ \\
Cavity & $\mathrm{TM}_{0 m}$ & $\epsilon_{r z}^{\prime}$ & $\pm 0.5 \%$ & $\pm 5 \times 10^{-4}$ \\
Dielectric reson. & $\mathrm{TE}_{01}$ & Very Accurate & \pm 0.2 & $\pm 5 \times 10^{-5}$ \\
Coax probe & $\mathrm{TEM}, \mathrm{TM}_{01}$ & Nondestructive & $\pm 2-10 \%$ & \pm 0.02 \\
Fabry-Perot & $\mathrm{TEM}$ & High frequency & $\pm 2 \%$ & \pm 0.0005 \\
\hline
\end{tabular}

techniques, one for the component of permittivity normal to specimen face and one for in-plane permittivity. The loss measurement is not usually affected as much as $\epsilon_{r}^{\prime}$ by anisotropy, and a single measurement of loss often suffices. For example, resonant transmission-line methods can be used for $\epsilon_{r}^{\prime}$ and the loss could be obtained by more accurate in-plane techniques such a $T E_{01}$ resonator. However, there are materials where the loss is significantly anisotropic.

Measurement of magnetic substrates requires a strong magnetic field. Magnetic materials can be measured by a split-post magnetic resonator, also by $\mathrm{TM}_{110}[28]$ or $\mathrm{TE}_{011}$ cavities, whispering-gallery modes, or $\mathrm{TE}_{011}$ cavities or dielectric resonators (see table 9).

The ASTM (American Society for Testing and Materials) and the IPC (formerly known as Institute for Interconnection and Packaging Electronic Circuits) standards 
Table 9. Magnetic measurement methods compared with typical uncertainties.

\begin{tabular}{c|cccc}
\hline Technique & Field & Advantages & $\Delta \mu_{r}^{\prime} / \mu_{r}^{\prime}$ & $\Delta \tan \delta_{r}$ \\
\hline Coaxial Line & $\mathrm{TEM} \mathrm{TE}_{10}$ & Broadband & $\pm 2 \%$ & \pm 0.01 \\
or waveguide & & & & \\
Cavity & $\mathrm{TE}_{011}$ & Very accurate & $\pm 0.5 \%$ & $\pm 5 \times 10^{-4}$ \\
Cavity & $\mathrm{TM}_{110}$ & $\mu_{r z}^{\prime}$ & $\pm 0.5 \%$ & $\pm 5 \times 10^{-4}$ \\
Dielectric reson. & $\mathrm{TE}_{011}$ & Very accurate & $\pm 0.5 \%$ & $\pm 5 \times 10^{-4}$ \\
Whispering-gallery & $\mathrm{Hybrid}$ & Very accurate & \pm 1 & $\pm 5 \times 10^{-6}$ \\
Courtney & $\mathrm{TE}_{01}$ & Very accurate & \pm 1 & $\pm 5 \times 10^{-5}$ \\
\hline
\end{tabular}

committees have developed a number of standard dielectric measurement techniques [29]. The techniques applicable to thin materials are summarized in tables 10 and 11. 
Table 10. ASTM standard techniques for dielectric measurements.

\begin{tabular}{c|ccc}
\hline ASTM No. & Applicability & Method & Frequency \\
\hline D150 & discs & capacitor & $1 \mathrm{~Hz}$ to $10 \mathrm{MHz}$ \\
D1531 & thin sheeting & bridge network & $1 \mathrm{kHz}$ to $1 \mathrm{MHz}$ \\
D1673-94 & polymers & capacitor & $10 \mathrm{kHz}$ to $100 \mathrm{MHz}$ \\
D2149 & discs & capacitor & $50 \mathrm{~Hz}$ to $10 \mathrm{MHz}$ \\
D2520 & small rods & rectangular resonator & $0.5 \mathrm{GHz}$ to $50 \mathrm{GHz}$ \\
D3380 & clad substrates & stripline & $8 \mathrm{GHz}$ to $12 \mathrm{GHz}$ \\
D5568 & cylindrical samples & coaxial line & $1 \mathrm{MHz}$ to $20 \mathrm{GHz}$ \\
\hline
\end{tabular}

Table 11. IPC standard techniques for dielectric measurements.

\begin{tabular}{c|ccc}
\hline IPC No. & Applicability & Method & Frequency \\
\hline $2.5 .5 \mathrm{~A}$ & clad substrates & capacitor & $0.01 \mathrm{MHz}$ to $1 \mathrm{MHz}$ \\
$2.5 .5 .2 \mathrm{~A}$ & clad substrates & capacitor & $0.01 \mathrm{MHz}$ to $1 \mathrm{MHz}$ \\
2.5 .5 .5 & clad substrates & stripline & $10 \mathrm{GHz}$ to $13 \mathrm{GHz}$ \\
2.5 .5 .5 .1 & clad substrates & stripline & $500 \mathrm{MHz}$ to $10 \mathrm{GHz}$ \\
2.5 .5 .6 & clad substrates & full-sheet resonance & $500 \mathrm{MHz}$ to $10 \mathrm{GHz}$ \\
2.5 .5 .1 & clad substrates & capacitor & $0.1 \mathrm{MHz}$ to $10 \mathrm{MHz}$ \\
2.5 .5 .2 & unclad substrates & capacitor & $0.1 \mathrm{MHz}$ to $10 \mathrm{MHz}$ \\
2.5 .5 .3 & unclad substrates & capacitor & $0.1 \mathrm{MHz}$ to $10 \mathrm{MHz}$ \\
2.5 .5 & unclad substrates & capacitor & $0.1 \mathrm{MHz}$ to $10 \mathrm{MHz}$ \\
2.5 .5 .8 & unclad films & capacitance & $.01 \mathrm{MHz}$ to $1 \mathrm{MHz}$ \\
2.5 .5 .9 & clad substrates & transmission line & $1 \mathrm{MHz}$ to $1.5 \mathrm{GHz}$ \\
\hline
\end{tabular}


Field orientation is important for measurements of anisotropic materials. The characterization of anisotropic materials generally requires two techniques, one for the component of permittivity perpendicular to the plane of the sample and one for the in-plane permittivity.

Measurement fixtures in which the electromagnetic fields are tangential to the air-material interfaces, such as in $\mathrm{TE}_{01}$ cavities and dielectric resonators, generally yield more accurate results than fixtures where the fields are normal to the interface. If the electric field is tangential to a gap between a material and a metallic wall of a measurement fixture, then the measurement of $\epsilon_{r}^{\prime}$ is minimally influenced by the gap and no gap correction is normally required. Unfortunately, for many applications it is not always possible or preferable to measure in-plane field orientations. For example, circuit boards and PWBs operate with the electric field primarily normal to the plane of the sheet and, therefore, this component of the permittivity is of primary interest. However, measurements with the electric field perpendicular to the sample face may suffer from consequences of air-gap depolarization. In such cases, the air gap must be either accepted, mitigated by metalization of sample surfaces or application of conductive pastes, or corrections made by numerical techniques $[4,5]$. The gap correction is due to the capacitance of air in the gap and effectively depends on frequency only through the dependence of $\epsilon_{r}$ in the gap-correction formula.

In the radio-frequency and microwave bands, $\epsilon_{r}^{\prime}$ is a monotonically decreasing function of increasing frequency (see figures 7 and 8). In the radio-frequency and low microwave bands, any increase in $\epsilon_{r}^{\prime}$ as the frequency increases is almost always due to a fixture geometrical resonance or other fixture-dependent resonance. For lossy materials $\epsilon_{r}^{\prime}$ decreases faster as frequency increases (dispersion) than it does in nonlossy materials. On the other hand, $\epsilon_{r}^{\prime \prime}$ does not necessarily decrease as frequency 


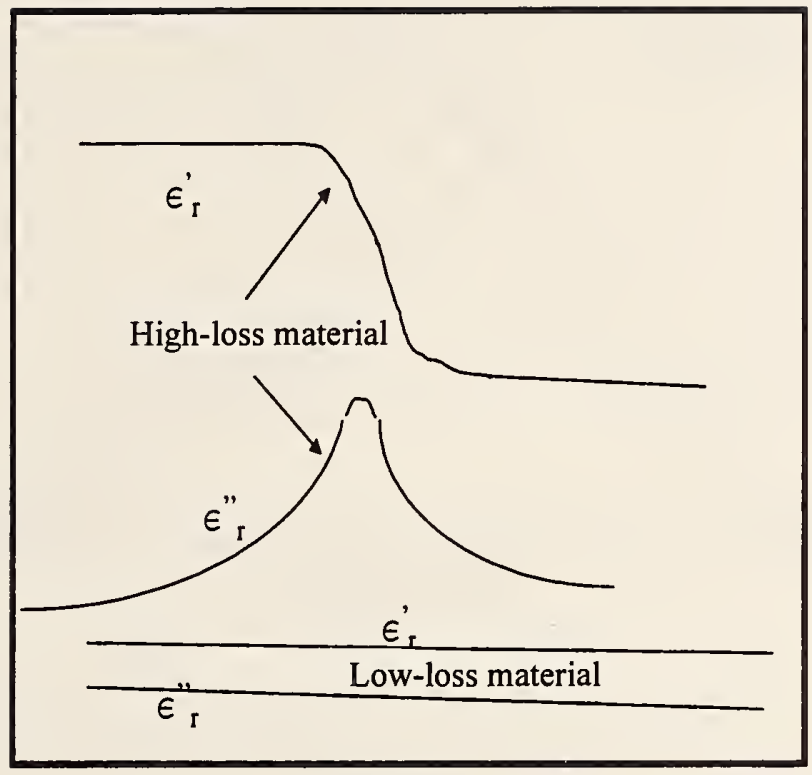

Frequency

Figure 7. Typical frequency dependence in low loss and Debye materials.

increases. It may increase as frequency increases or form a Debye loss peak. The loss tangent $\left(\tan \delta=\epsilon_{r}^{\prime \prime} / \epsilon_{r}^{\prime}\right)$ of most ceramics increases nearly linearly as frequency increases; in such cases, it is possible to extrapolate measurement data from one frequency range to another. Materials of very low-loss have loss tangents in the microwave spectrum that are nearly frequency independent. In materials with a finite dc conductivity, $\epsilon_{r}^{\prime \prime}$ increases at low frequencies as $1 / \omega$, where $\omega$ is radial frequency, (for example, $<1 \mathrm{kHz}$ ). Conducting materials are difficult to measure at low frequencies $(f<1 \mathrm{MHz})$. This is because the boundary layer between the sample and electrode produces an electrode-polarization capacitance that must be removed from the measurement result. 


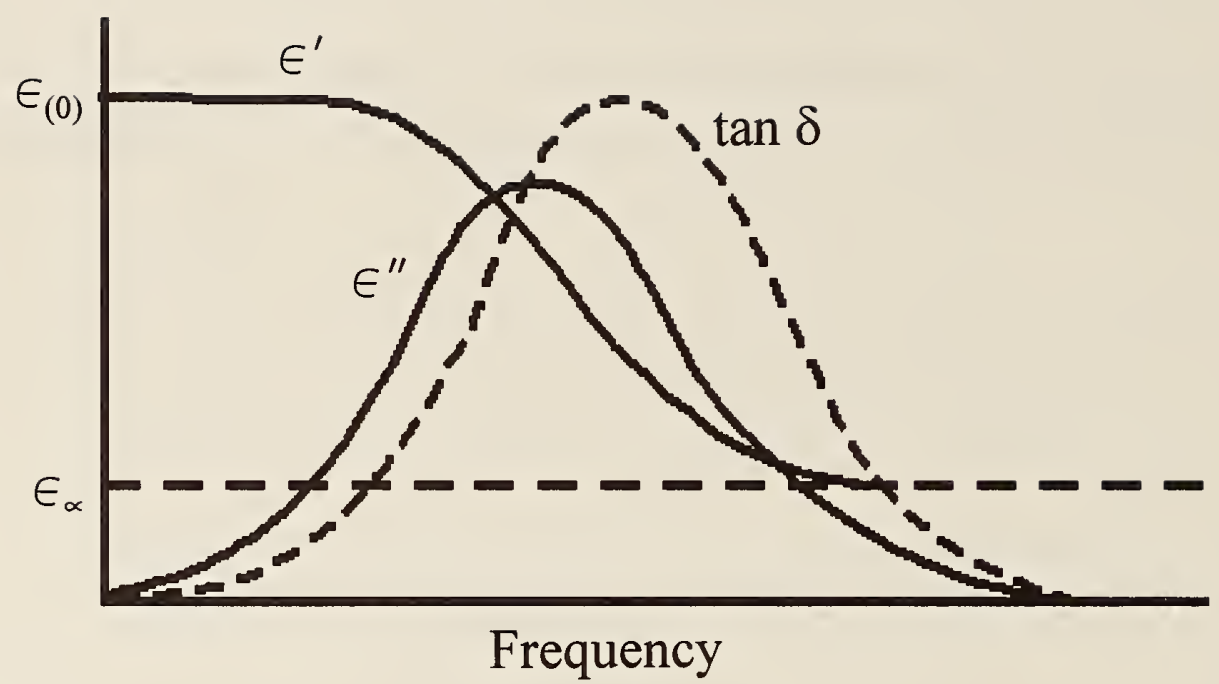

Figure 8. Typical permittivity variation for polar materials.

The real and imaginary parts of the permittivity, excluding dc conductivity, $\sigma / \omega$ in $\epsilon_{r}^{\prime \prime}$, are related by Kramers-Kronig dispersion relations [30]. This is a result of causality and yields a relationship between the real and imaginary parts of the permittivity. A consequence of these relations is that frequency-dependent changes in the real and imaginary parts of the permittivity are correlated (see, for example, the correlation in figure 7).

There are many models used for extracting permittivity and permeability. Methods based on Maxwell's equations are single or full-mode models that relate the resonant frequency or transmission and reflection coefficients to functions of the permittivity (see table 12). These types of models are accurate, but require complicated solutions to Maxwell's equations and can be computationally intensive.

At lower frequencies, dielectric and magnetic material properties can be extracted from a transmission-line model using the impedance $Z_{c}$ and propagation constant $\gamma$ 
Table 12. Permittivity models

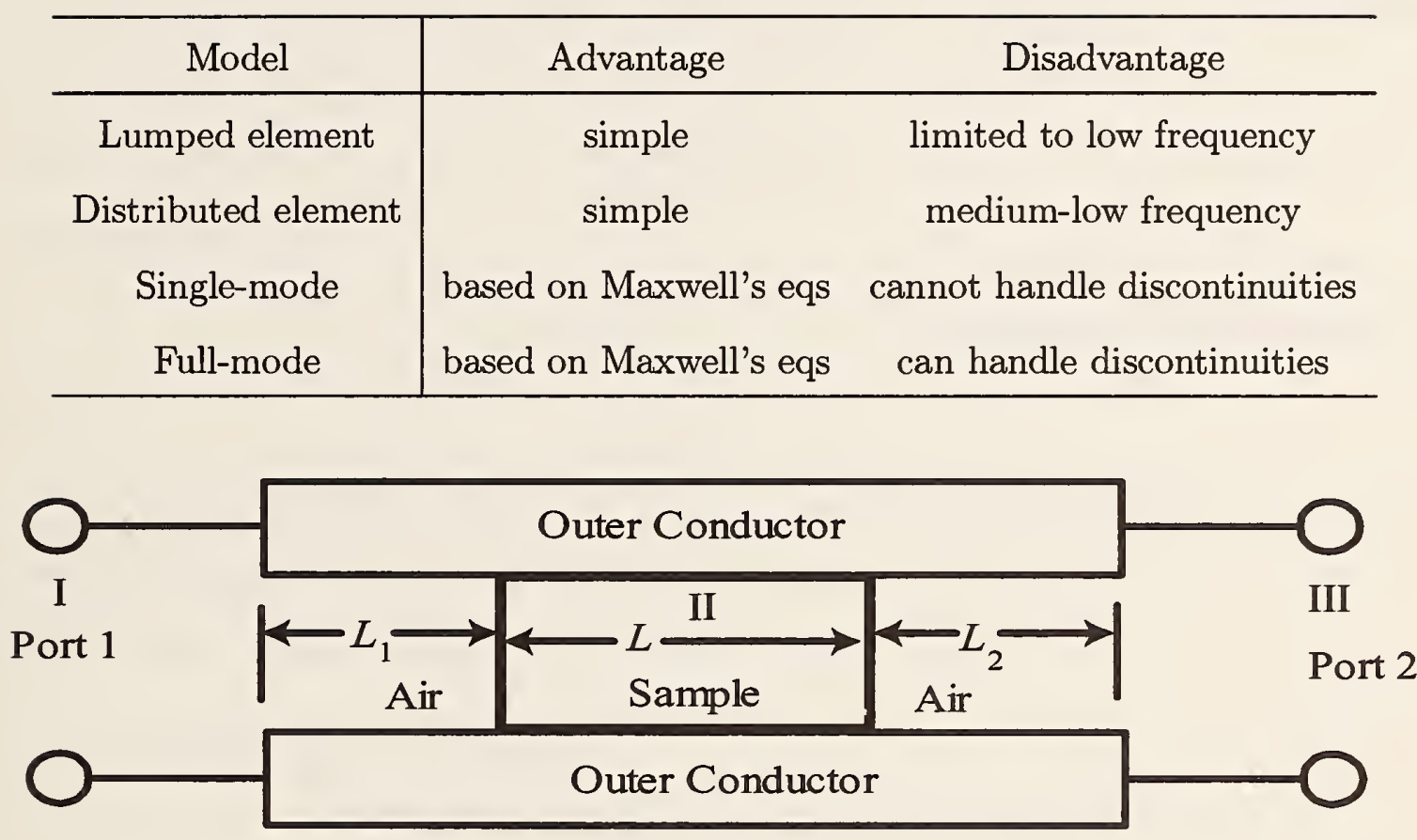

Figure 9. waveguide transmission line with sample.

(see figure 9). For cases where both dielectric and magnetic properties are required one must use both the impedance and propagation constant simultaneously [5, 27].

For dielectrics, either the impedance or propagation constant may be used in isolation for determination of the permittivity. In both cases, a model of the transmissionline structure is required that relates the material properties to the reflection and transmission coefficients. The models may be based on lumped circuits or distributed elements. For distributed-element models, usually an approximation to Maxwell's equations in terms of current and voltage waves in the transmission line are used.

We now overview the basic approach for determining the permittivity or permeability for a filled transmission line (see, for example, in reference [27]). We will 
go into more detail in a later section for rectangular waveguide and coaxial line measurement methods. The voltage reflection coefficient is

$$
\rho_{V}=\frac{Z_{C}-Z_{0}}{Z_{C}+Z_{0}}
$$

where $Z_{0}$ is the impedance of the empty transmission line, typically $50 \Omega$. This equation can be rewritten as

$$
Z_{C}=\frac{1+\rho_{V}}{1-\rho_{V}} Z_{0}
$$

The permittivity (permeability) can be found by solving eq (5), given the permeability (or permittivity) of the transmission line and then using it in a model for the transmission line that relates material properties to the characteristic impedance of the transmission line.

A transmission line of length $L$ has a transmission coefficient

$$
T_{L}=\exp (-\gamma L)
$$

where $\gamma=\alpha+j \beta$ is the propagation constant. It is possible to use two or more lengths of identical transmission lines of lengths $L_{i}$ to extract the permittivity and permeability. Or one can use both the impedance and propagation constant equations to obtain four independent equations for the four electrical parameters, as is done in scattering-matrix approaches [5].

The attenuation constant is computed from

$$
\alpha=\frac{1}{L_{1}-L_{2}} \ln \left[\frac{T_{L 2}}{T_{L 1}}\right] .
$$

The permittivity can be determined from the phase, which is calculated from

$$
\beta=\frac{1}{\Delta L} \arctan \left[\frac{\operatorname{Im}\left(e^{\alpha \Delta L} e^{-\gamma \Delta L}\right)}{\operatorname{Re}\left(e^{\alpha \Delta L} e^{-\gamma \Delta L}\right)}\right] .
$$


Due to the inverse tangent, this function is multivalued. Therefore, the correct physical root must be determined. Frequently the transmission line has more than one dielectric material. In these cases, an effective permittivity is measured and a mixture equation may be used to separate the various component permittivities (see Appendix).

Surface roughness of the conductor increases the capacitance, resistance, and inductance. The capacitance is increased because the effective thickness of the rough material between two conductors is less than the measured thickness [31]. The roughness of the surface of the conductors can also affect the resistance due to the increased surface area.

\subsection{Resonant Methods}

In this section, we overview the most commonly used resonant measurement techniques for unmetalized thin materials. We discuss and compare the strengths and weaknesses of each technique. We will also compare the techniques in light of destructive versus nondestructive measurements. For each technique, we either overview the theory or refer to published work. We will indicate which fixtures can be used for obtaining permittivity in or out of the plane of the sample. We also give an uncertainty range on $\epsilon_{r}^{\prime}$ and $\tan \delta$ for each fixture. For thin films, we refer the interested reader to recent publications [32-34].

For resonant measurements, the permittivity or permeability is determined from measurements of the resonance frequency and quality factor $(\mathrm{Q})$ as shown in figure 10. The quality factor is calculated from $Q=f_{0} / \Delta f$, where $f_{0}$ is the resonance frequency and $\Delta f$ is the frequency difference between $3 \mathrm{~dB}$ points. 


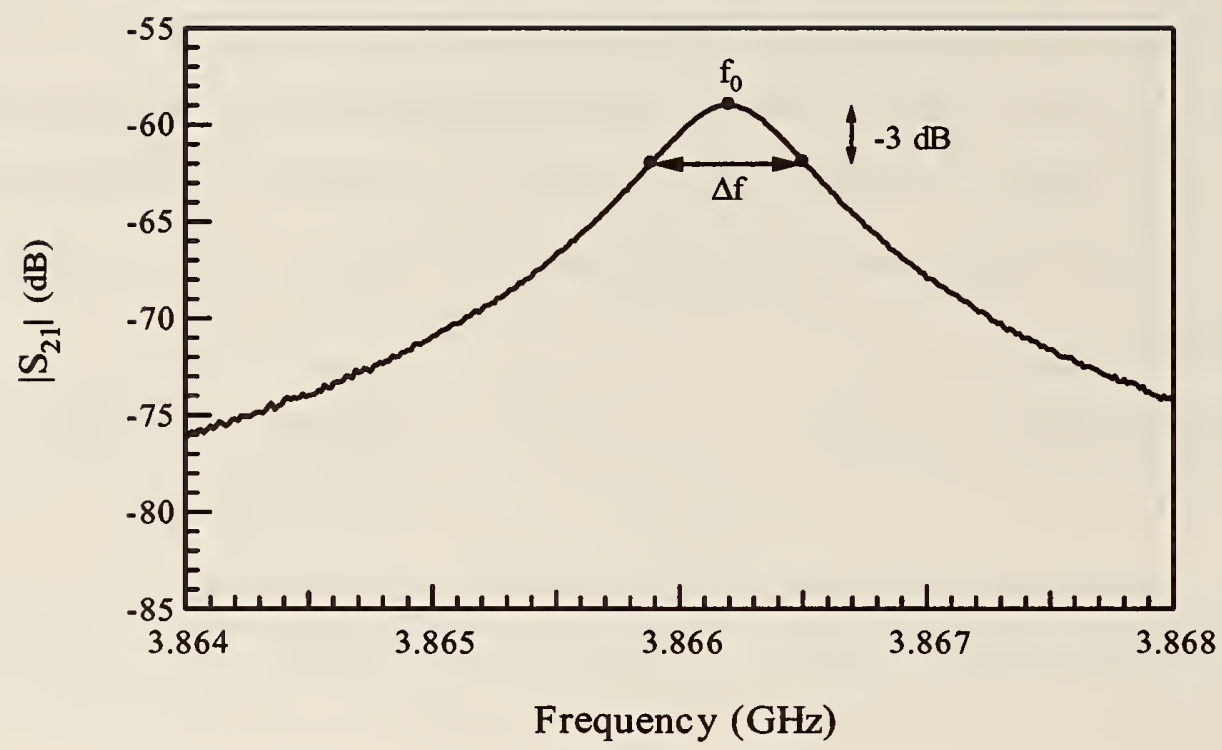

Figure 10. Measuring resonant frequency and Q.

\subsubsection{Reentrant Cavity}

Resonant measurement methods are the most accurate ways of obtaining permittivity and loss tangent. Most resonant techniques yield permittivity in the plane of the sample. The resonant reentrant cavity is an exception in that it yields the permittivity component normal to the face of the material at frequencies from 100 $\mathrm{MHz}$ to $1 \mathrm{GHz}$.

The reentrant cavity depicted in figure 11 consists of a coaxial line or other transmission line with a gap in the inner electrode. The specimen is inserted into this gap. The cavity is then resonated and the capacitance of the gap produces a frequency shift. If the specimen gap region is at the very top or bottom of the cavity then the system is called a singly reentrant cavity, whereas, if the sample gap is in the middle of the cavity it is called a doubly reentrant cavity. The loss tangent is determined from quality-factor measurements with and without the specimen in place. 


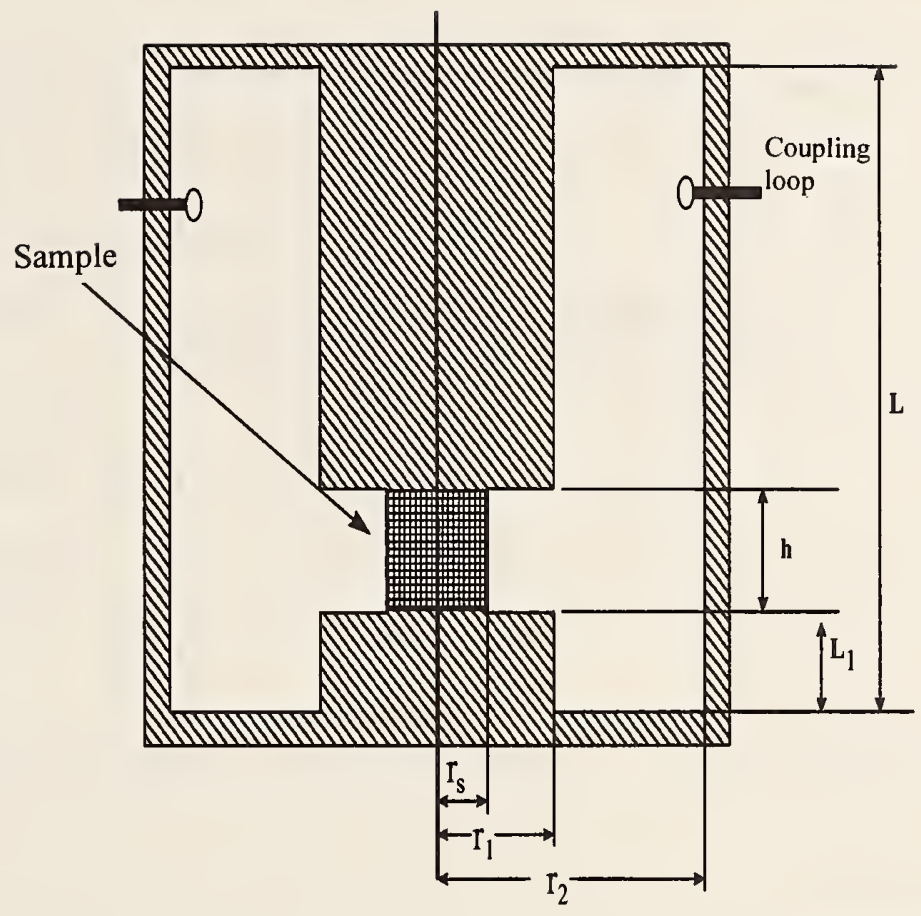

Figure 11. Schematic cross section of the reentrant cavity. The coaxial line inner and outer diameters are $2 r_{1}$ and $2 r_{2}$, and specimen diameter is $2 r_{s}$, as indicated. The cavity height is $L$.

Typical expanded uncertainties (U) (with coverage factor $k=2$ (number of standard deviations) [35], for a well-characterized system for low to medium permittivity are $U_{\epsilon}=0.01$, and for loss tangent $U= \pm 0.001$.

Two approaches have been used for modeling the reentrant cavity. The first is a lumped-circuit approximation [36,37]. The other approach is a rigorous modematching technique [38-40]. The full-field model is given in reference [38]. The software for this fixture is usually limited to samples greater than $1 \mathrm{~mm}$ in thickness. 


\subsubsection{Split-Cylinder Resonators}

The split-cylinder resonator is used to nondestructively measure the complex permittivity of low-loss, unclad dielectric materials at microwave frequencies. This method uses a cylindrical cavity which is separated into two halves as shown in Figure 12. A sample is placed in the gap between the two shorted cylindrical waveguide sections. A $T E_{011}$ resonance is excited with the coupling loops, and from measurements of the resonant frequency and the quality factor, one determines the complex permittivity of the sample. Generally, the resonator is operated such that the $\mathrm{TE}_{01}$ mode propagates within the sample and is evanescent outside the sample. Note that for this mode the electric field is in the plane of the sample.

In terms of sample geometry, the only requirements are that the sample must extend beyond the diameter of the two cavity sections and the sample must be flat. This provides the accuracy of a resonator technique without having to machine the sample. Typical uncertainties are for the real part of the permittivity $U= \pm 0.005$ and loss tangent $U= \pm 5 \times 10^{-5}, k=2$.

The original theory for this technique is summarized in references [41-43]. However, an improved theoretical model, based on the mode-matching method exists that properly takes into account the fringing fields in the sample region outside of the cylindrical waveguide sections [44].

\subsubsection{Cavity Resonators}

Cavities are also used to measure the permittivity of thin materials. The most common cavity is the $\mathrm{TE}_{01}$ resonator [45]. In this case, the permittivity in the plane of the specimen is measured. In order to obtain a stronger electric field, the sample usually is elevated above the cavity floor. $\mathrm{The}^{\mathrm{T}} \mathrm{TM}_{0 m}$ cavity is also commonly 


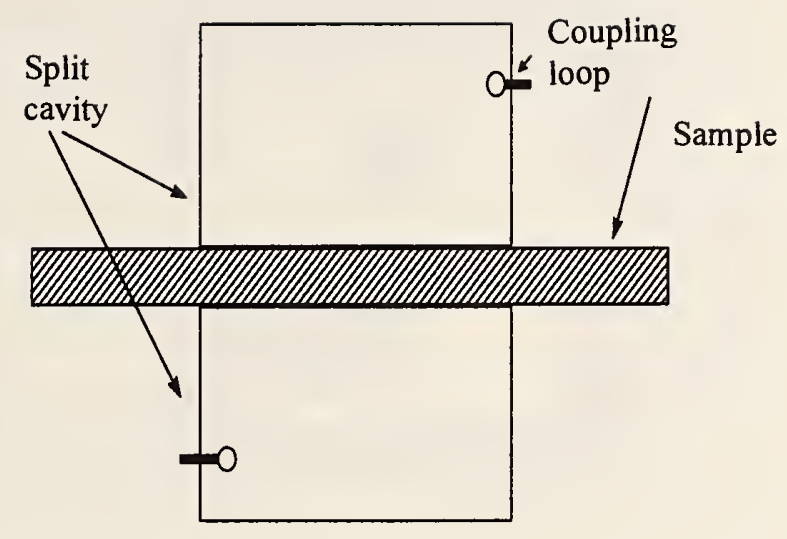

Figure 12. $\mathrm{TE}_{01}$ split-cylinder resonator for thin dielectrics.

used. In this cavity, there is a strong $E_{z}$ component of the electric field at the center. If a specimen is placed near the center, then it is possible to measure the $E_{z}$ component of the permittivity [46]. Either rod or bulk specimens can be used. In these measurements, a thin rod specimen is inserted into a hole at the center of the cavity [28]. A rod specimen has an advantage of lower depolarization effects than with a flat sample.

Rectangular resonators are also commonly used (see ASTM test method 2520). In this method a rod sample is inserted into a waveguide and a perturbation model is used to obtain the permittivity. For accurate measurement with this technique, a correction for the hole in the waveguide is necessary.

\subsection{4 $\mathrm{TE}_{01}$ Split-Post Dielectric and Magnetic Resonators}

The split-post dielectric and magnetic resonator techniques are methods for measurement of thin materials (see figures 13 and 14) [47-49]. In the split-post method, 


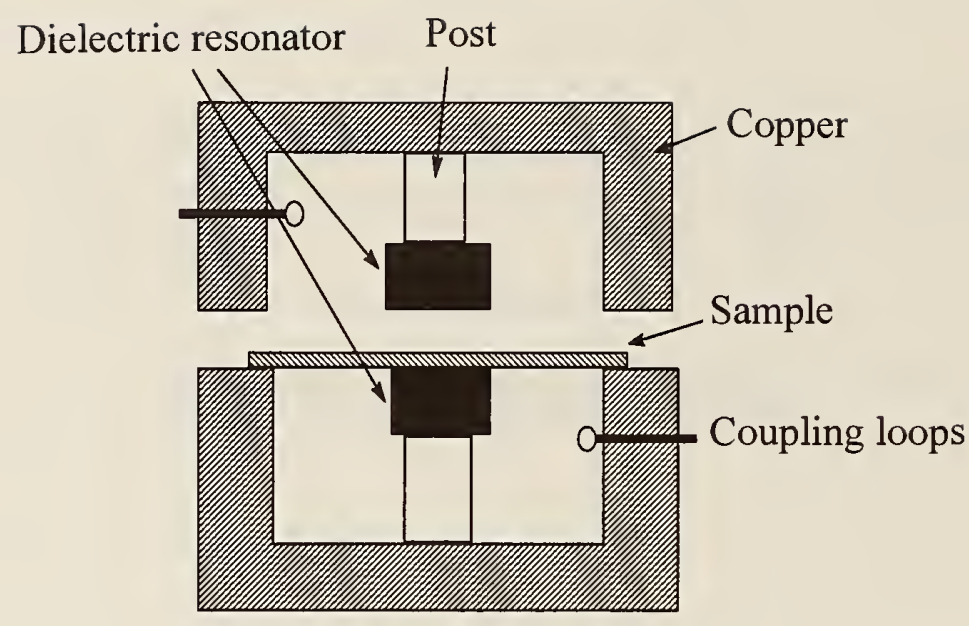

Figure 13. Schematic diagram of a split dielectric resonator fixture.

a thin material or film is inserted between two fixed dielectric resonators. The posts that support the resonators have low permittivity and low loss. The quality factor of the support post is influenced by the presence of the specimen. Split dielectric resonators were developed by Nishikawa et al. [48], Maj [49], and DelaBalle [50]. There are many variations of this technique reported in the literature. Some fixtures have the specimen extending to the back wall of the cavity. The lowest-loss technique is when the dielectric resonators are supported by low-permittivity posts. This configuration has the advantage of reduced wall loss. Various techniques have been employed for analyzing this method: finite difference, mode-matching, and finite-element methods [48].

The geometry of a split dielectric resonator fixture used in our measurements is shown in figure 13. Such a resonator operates with the $\mathrm{TE}_{01}$ mode, which has only an azimuthal electric field component so that the electric field remains continuous across the dielectric interfaces. This also minimizes systematic air-gap uncertainties. 


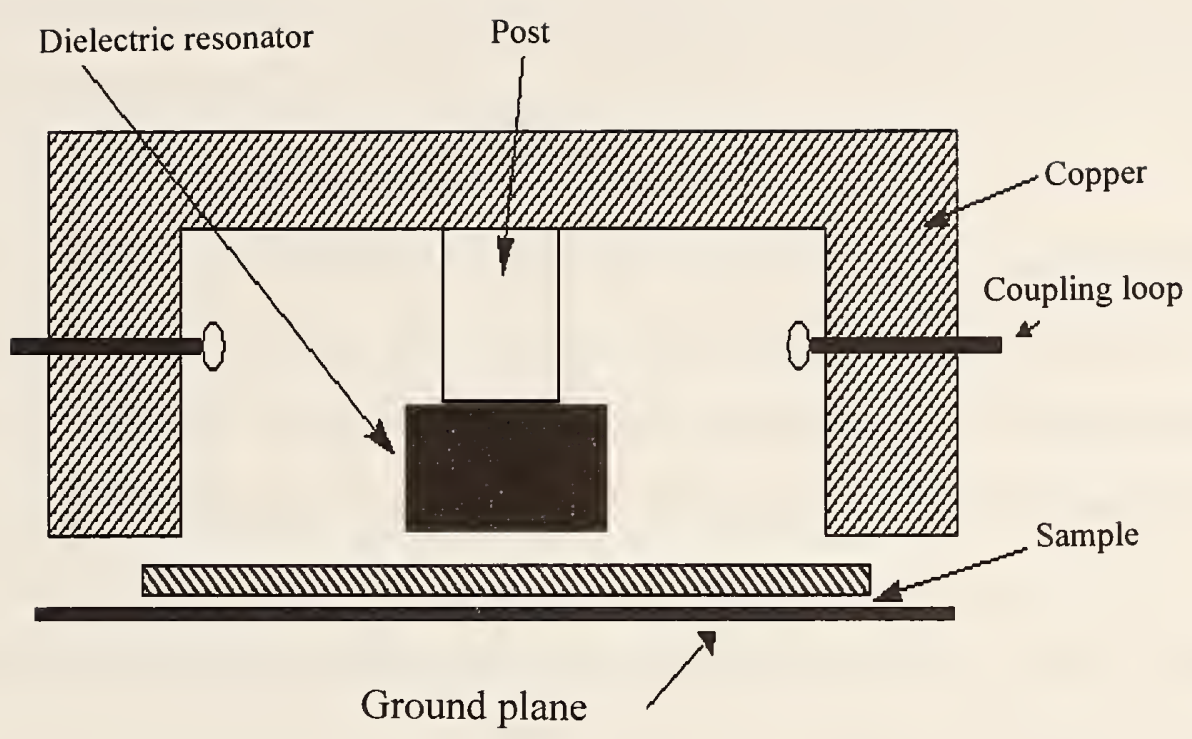

Figure 14. Schematic diagram of a post magnetic resonator fixture.

The resonator measures the permittivity component in the plane of the specimen. A useful feature of the split-post resonator is that it can be designed to operate in the low gigahertz region. We have successfully used dielectric resonators operating from $1.5 \mathrm{GHz}$ to $12 \mathrm{GHz}$. Loop coupling is used in these dielectric resonators.

Thin magnetic materials can be measured with the magnetic post resonator technique as shown in figure 14. This fixture consists of a dielectric post as shown in figure 14 above a ground plane. The ground plane induces a region of strong magnetic field. The thin magnetic material is inserted between the posts and then the resonance frequency and quality factors are measured. The same sample can also be measured with the split-post resonator. From these two measurements it is possible to solve simultaneously for both $\epsilon$ and $\mu$.

The main source of uncertainty in $\epsilon_{r}^{\prime}$ in the split-post, as with the split-cylinder resonator measurement, is from the uncertainty in the thickness of the specimen. We have found that the following equation is useful in estimating the relative uncertain- 
ties $U_{\epsilon}, k=1$, in $\epsilon_{r}^{\prime}, \Delta \epsilon_{r}^{\prime}$ for a specimen of thickness $L$ :

$$
U \approx 2 \frac{\Delta L}{L}
$$

Typical uncertainties in the split-post resonator are $U_{\epsilon(r)}= \pm 0.002$. Here $U_{\epsilon(r)}$, $k=2$, is the expanded uncertainty [35]. The uncertainty in the dielectric loss tangent depends on other factors such as the accuracy with which the quality factor can be measured. The uncertainty in the dielectric loss tangent is usually absolute, but is relative for $\epsilon_{r}^{\prime}$. For a properly chosen specimen thickness, it is usually possible to resolve dielectric loss tangents with uncertainty $U= \pm 2 \times 10^{-5}$ for quality-factor measurements made with accuracies of $\pm 2 \%$.

\subsubsection{Courtney Technique}

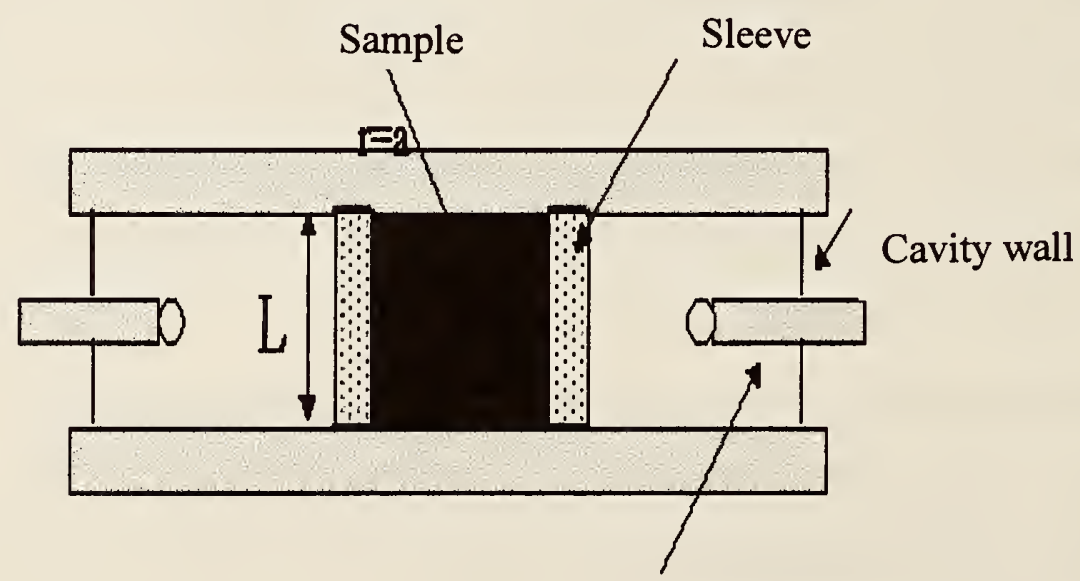

Coupling loop

Figure 15. Courtney fixture with rod sample.

The Courtney fixture, as shown in figure 15, can be used to measure the dielectric properties of materials at gigahertz frequencies [51-57]. In this technique, the $\mathrm{TE}_{01 \delta}$ 
mode is used to determine the dielectric properties. For a dielectric measurement, a rod sample is placed between plates, and the resonante frequency and $\mathrm{Q}$ are measured. Low-loss, high-permittivity sleeves can be used around the sample to lower the resonante frequency, thereby increasing the frequency coverage. If the material is magnetic, two measurements are required to get both the permittivity and permeability of a material. First, the material is measured in the resonator without an applied magnetic field and then the Courtney fixture is placed between the poles of a magnet so that both $\epsilon_{r}^{\prime}$ and $\mu_{r}$ can be determined.

For a rod resonator (without a sleeve), of height $L$, in a cavity with walls at position $r=b$, the following transcendental equation can be solved for $\epsilon_{r}^{\prime}[58,59]$ in terms of Bessel functions $J_{1}$ and modified Bessel functions $K_{1}$ and $I_{1}$ :

$$
\frac{J_{1}\left(k_{s} a\right)\left(K_{0}\left(k_{s} a\right)-\frac{K_{1}\left(k_{s} b\right)}{I_{1}\left(k_{s} b\right)} K_{0}\left(k_{s} a\right)\right)}{k_{s} a}+\frac{\left(K_{1}\left(k_{s} a\right)-\frac{K_{1}\left(k_{s} b\right)}{I_{1}\left(k_{s} b\right)} I_{1}\left(k_{s} a\right)\right) J_{0}\left(k_{s} a\right)}{k_{a} a}=0
$$

where

$$
\begin{aligned}
& k_{a}=\sqrt{\frac{\omega^{2}}{c^{2}}-\left(\frac{\pi}{L}\right)^{2}} \\
& k_{s}=\sqrt{\frac{\omega^{2} \epsilon_{r}^{\prime}}{c^{2}}-\left(\frac{\pi}{L}\right)^{2}} .
\end{aligned}
$$

$E_{\phi}$ is assumed to vanish at $r=b$. The quality factor is related to losses in the sample and conductors

$$
\frac{1}{Q}=\frac{1}{Q_{0, c}}+\frac{1}{Q_{0, d}}
$$

where

$$
Q_{0, c}=\frac{240 \pi^{2} \epsilon_{r}}{R_{s}}\left(\frac{L}{\lambda}\right)^{3} \frac{1+R}{1+\epsilon_{r} R}
$$




\section{Coupling loops}

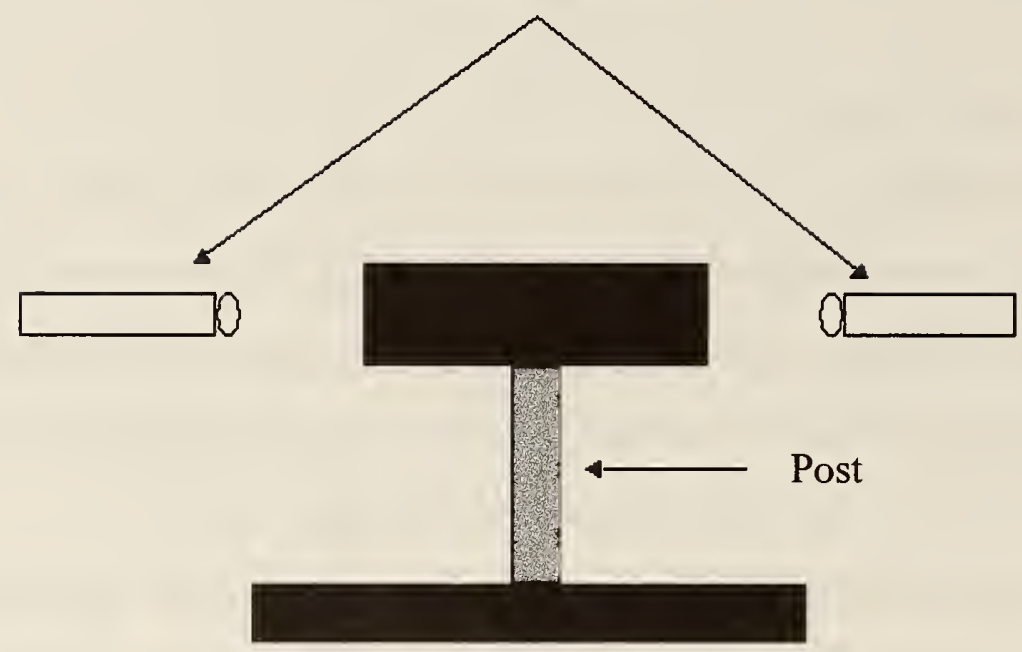

Figure 16. Whispering gallery mode resonator. The specimen is in form of a disk.

and $R_{s}$ is surface resistance of the end plates.

$$
Q_{0, d}=\frac{1+R}{\tan \delta_{s}},
$$

where $\tan \delta_{s}$ is the loss tangent of the rod and

$$
R=\left[\frac{1}{\epsilon_{r}} \frac{k_{s} J_{0}\left(k_{s} a\right)}{k_{a}\left(K_{0}\left(k_{a} a\right)-\frac{K_{1}\left(k_{a} b\right)}{I_{1}\left(k_{a} b\right)} I_{0}\left(k_{a} a\right)\right)}\right]^{2} \frac{\int_{a}^{b}\left(K_{1}\left(k_{a} r\right)-\frac{K_{1}\left(k_{a} b\right)}{I_{1}\left(k_{a} b\right)} I_{1}\left(k_{a} r\right)\right)^{2} r d r}{\int_{0}^{a} J_{1}^{2}\left(k_{s} r\right) r d r}
$$

In addition, there are metal losses on the cavity outer wall located at $r=b$.

\subsubsection{Whispering-Gallery Resonators}

Low-loss dielectric and magnetic materials can be measured in the microwave frequency range by use of whispering-gallery mode resonators as shown in figure 16 [60-63]. Whispering-gallery modes have been used in measurements of both $\epsilon_{r}^{\prime}$ and $\tan \delta$. Highly accurate measurements of dielectric loss can be made using this technique. In this dielectric-resonator technique, the specimen is usually supported from 
the bottom with a dielectric rod. The support rod does not unduly influence the measurement because the modes do not penetrate appreciably into the rod $[50,64-69]$. Measurements using whispering-gallery modes on low-loss materials are advantageous since conductor loss can be made very small.

\subsubsection{Fabry-Perot Resonators}
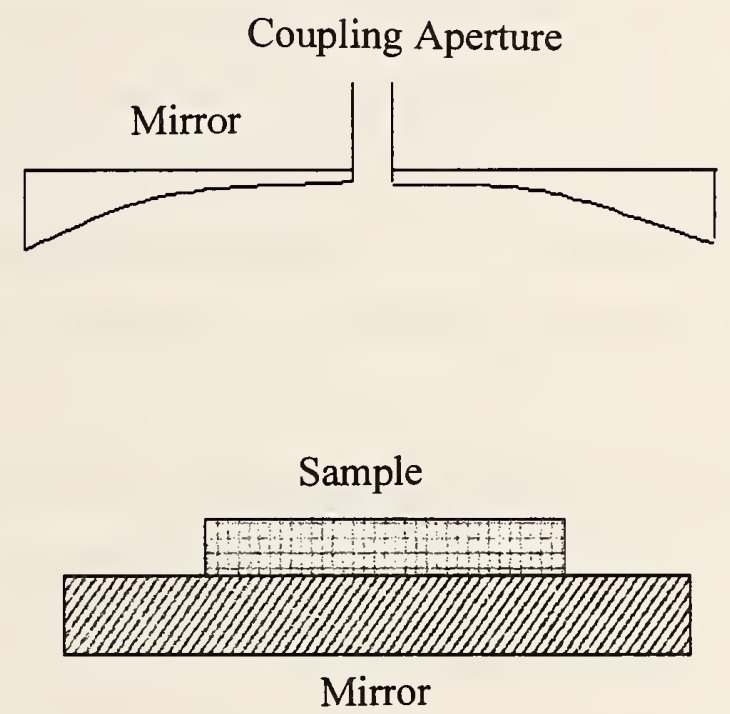

Figure 17. Fabry-Perot resonator with specimen on the post.

Open resonators have been used for measuring low-loss materials in the millimeter range in the configuration shown in figure 17 [70-74]. Open Fabry-Perot resonators consist of two separated mirrors with a coupling aperture on one of the mirrors. In the confocal setup both mirrors are concave, whereas in the semi-confocal arrangement only one of the mirrors is concave and the other is flat (see figure 17). Since they are open structures they suffer from leakage of radiation. The concave feature of the mirror minimizes radiation leakage from the open sides of the resonator because it focuses the beam onto a smaller area of the specimen under test, thereby minimizing 
specimen edge diffraction effects. Fabry-Perot resonators have large quality factors, typically 20,000 to 100,000 , and are useful for measurements on thin, low-loss materials. The metal in the mirrors limits the accuracy of the loss measurement. The tensor permittivity values can be obtained by measuring at different angles of incidence. Thin substrate materials can be measured with this technique by positioning the specimen on the flat mirror. The permittivity is found by solving [75]

$$
\tan \left(n k t-\Phi_{t}\right)+n \tan \left(k d-\Phi_{d}\right)=0
$$

where $n=\sqrt{\epsilon_{r s}}, d=D-t, \Phi_{t}=\arctan \left(t / n z_{0}\right), \Phi_{d}=\arctan \left(d^{\prime \prime} / z_{0}\right)-\arctan \left(t / n^{2} z_{0}\right)$, $z_{0}=\sqrt{d^{\prime \prime}\left(R_{0}-d^{\prime \prime}\right)}, d^{\prime \prime}=d+t / n^{2}, D$ is distance between mirrors, $t$ is sample thickness, $R_{0}$ is radius of curvature of the mirror, $w$ is the beam width, and $k=2 \pi / \lambda$, where $\lambda$ is the wavelength in free space.

A correction for the flat mirror in a Gaussian beam is given by

$$
\frac{\Delta f}{f}=-\frac{n^{2}-1}{n^{4}} \frac{t \Delta \sin ^{2}\left(n k t-\Phi_{t}\right)}{k^{2} w^{2}(t \Delta+d)}
$$

where

$$
\Delta=\frac{n^{2}}{n^{2} \cos ^{2}\left(n k t-\Phi_{t}\right)+\sin ^{2}\left(n k t-\Phi_{t}\right)} .
$$

The loss tangent is found from

$$
\tan \delta=\frac{2 n k t(d+t \Delta)}{Q_{e} \Delta\left(2 n k t-\sin \left(n k t-\Phi_{t}\right)\right)}
$$

and the quality factor is

$$
\frac{1}{Q_{e}}=\frac{1}{Q_{d}}+\frac{1}{Q_{l}},
$$

where $Q_{d}$ is quality factor with the sample present and

$$
Q_{l}=Q_{0} \frac{2(t \Delta+d)}{D(\Delta+1)},
$$

where $Q_{0}$ is the quality factor without the sample present. 


\subsection{Transmission-Line Methods}

\subsubsection{Coaxial Lines and Waveguides: Transmission/Reflection and Short- Circuited Line}

Coaxial lines and waveguides are commonly used to measure samples of medium to high loss $[5,76-78]$ (see figure 9). A coaxial line of $7 \mathrm{~mm}$ diameter can be used to measure samples from $1 \mathrm{MHz}$ to approximately $18 \mathrm{GHz}$. A coaxial line of 14 $\mathrm{mm}$ diameter can be used to measure samples from $100 \mathrm{kHz}$ to $2 \mathrm{GHz}$. X-band waveguides can be used to measure from $10 \mathrm{GHz}$ to $13 \mathrm{GHz}$. Waveguide bands of other frequencies can be used to measure samples; however samples become large in the low gigahertz region. This technique is limited by air-gap effects to use with low-to-medium dielectric constants unless the sample surfaces in contact with the waveguide walls are metalized.

In two-port simultaneous measurements of permittivity and permeability, a sample is inserted into either a waveguide or a coaxial line, and the sample is subjected to an incident electromagnetic field. The scattering equations are found from an analysis of the electric field at the sample interfaces. In developing the scattering equations usually only the fundamental waveguide mode is assumed to exist.

As a special case, consider a perfectly dimensioned sample in a perfectly dimensioned waveguide as indicated in figure 9 . In this case no mode conversion occurs because the eigenfunctions in the air and sample regions are orthogonal with respect to cross-sectional coordinates. Therefore the modes may be decoupled, and the evanescent modes are not of concern. For a 2-port device the expressions for the measured scattering parameters are obtained by solving the boundary value problem subject to boundary conditions. The scattering parameters are defined in terms of the reflection coefficient $\Gamma$ and transmission coefficient $Z$ by: 


$$
\begin{gathered}
S_{11}=R_{1}^{2}\left[\frac{\Gamma\left(1-Z^{2}\right)}{1-\Gamma^{2} Z^{2}}\right], \\
S_{22}=R_{2}^{2}\left[\frac{\Gamma\left(1-Z^{2}\right)}{1-\Gamma^{2} Z^{2}}\right], \\
S_{21}=R_{1} R_{2}\left[\frac{Z\left(1-\Gamma^{2}\right)}{1-\Gamma^{2} Z^{2}}\right],
\end{gathered}
$$

where transmission coefficients are

$$
\begin{aligned}
& R_{1}=\exp \left(-\gamma_{o} L_{1}\right), \\
& R_{2}=\exp \left(-\gamma_{o} L_{2}\right),
\end{aligned}
$$

are the respective reference plane transformations and

$$
\gamma_{0}=j \sqrt{\left(\frac{\omega}{c_{l a b}}\right)^{2}-\left(\frac{2 \pi}{\lambda_{c}}\right)^{2}},
$$

where $c_{v a c}$ and $c_{l a b}$ are respectively the speed of light in vacuum and laboratory, and $\lambda_{c}$ is the cutoff wavelength. Also the transmission coefficient $Z$ is

$$
Z=\exp (-\gamma L)
$$

where $L$ is the sample length and

$$
\gamma=j \sqrt{\frac{\omega^{2} \mu_{r}^{*} \epsilon_{r}^{*}}{c_{v a c}^{2}}-\left(\frac{2 \pi}{\lambda_{c}}\right)^{2}},
$$

and a reflection coefficient

$$
\Gamma=\frac{\frac{\mu}{\gamma}-\frac{\mu_{0}}{\gamma_{0}}}{\frac{\mu}{\gamma}+\frac{\mu_{0}}{\gamma_{0}}}
$$


where $\epsilon_{r}^{*}$ and $\mu_{r}^{*}$ are the complex permittivity and permeability. For coaxial line, $\Gamma$ reduces to

$$
\Gamma=\frac{\frac{c_{v a c}}{c_{l a b}} \sqrt{\frac{\mu_{r}^{*}}{\epsilon_{r}^{*}}}-1}{\frac{c_{v a c}}{c_{l a b}} \sqrt{\frac{\mu_{*}^{*}}{\epsilon_{r}^{*}}}+1} .
$$

For nonmagnetic materials, eqs (23), (24), (25) contain $\epsilon_{r}^{\prime}, \epsilon_{r}^{\prime \prime}, L$, and the reference plane transformations $R_{1}, R_{2}$ as unknown quantities. For magnetic materials, we have the complex permeability as an additional unknown. Additionally, the total sample holder length $L_{\text {air }}=L+L_{1}+L_{2}$ is assumed known. We have measurements at each frequency point of all S-parameters. Therefore we have an over-determined system of equations. This abundance of information can be exploited by using optimization data reduction algorithms.

For dielectric materials we generally solve the following equation

$$
S_{11} S_{22}-S_{21} S_{12}=\exp \left\{-2 \gamma_{0}\left(L_{a i r}-L\right)\right\} \frac{\Gamma^{2}-Z^{2}}{1-\Gamma^{2} Z^{2}}
$$

for the permittivity in an iterative fashion $[27,76]$.

For magnetic materials, in order to obtain both the permittivity and the permeability from the scattering parameter relations, we need at least two independent measurements $[7,79]$. These independent measurements could involve two samples of different lengths or they could use a full 2-port measurement on a single sample. In the full S-parameter solution, we solve equations that are invariant to reference planes for $\epsilon$ and $\mu$. A set of equations for single-sample magnetic measurements is eq (33) in combination with

$$
\left(S_{21}+S_{12}\right) / 2=\exp \left\{-\gamma_{0}\left(L_{a i r}-L\right)\right\} \frac{Z\left(1-\Gamma^{2}\right)}{1-\Gamma^{2} Z^{2}}
$$


We can also obtain an explicit solution to eqs (33) and (34). Let $x=\left(S_{21} S_{12}-\right.$ $\left.S_{11} S_{22}\right) \exp \left\{2 \gamma_{0}\left(L_{a i r}-L\right)\right\}$ and $y=\left\{\left(S_{21}+S_{12}\right) / 2\right\} \exp \left\{\gamma_{0}\left(L_{a i r}-L\right)\right\}$; then it can be shown that the physical roots for the transmission coefficient for $|Z| \leq 1$ are [79]

$$
Z=\frac{x+1}{2 y} \pm \sqrt{\left(\frac{x+1}{2 y}\right)^{2}-1} .
$$

The reflection coefficient is

$$
\Gamma_{2}= \pm \sqrt{\frac{x-Z^{2}}{x Z^{2}-1}}
$$

The ambiguity in the plus-or-minus sign in eq (36) can be resolved by considering the reflection coefficient calculated from $S_{11}$ alone

$$
\Gamma_{3}=\frac{\eta\left(Z^{2}-1\right) \pm \sqrt{\eta^{2} Z^{4}+2 Z^{2}\left(2 S_{11}-\eta^{2}\right)+\eta^{2}}}{2 S_{11} Z^{2}},
$$

where $\eta=\exp \left(-2 \gamma_{0} L_{1}\right)$. The correct root for $\Gamma_{3}$ is picked by requiring $\left|\Gamma_{3}\right| \leq 1$. An estimate of $L_{1}$ is needed in eq (37). If $\Gamma_{2}$ is compared with $\Gamma_{3}$, then the plusor-minus sign ambiguity in eq (36) can be resolved and therefore $\Gamma_{2}$ is determined. The permeability and permittivity are then

$$
\mu_{r}^{*}=-\left(\frac{1+\Gamma_{2}}{1-\Gamma_{2}}\right) \frac{1}{\gamma_{0} L}(\ln Z+2 \pi j n)
$$

where $n=0, \pm 1, \pm 2 \ldots$ Also

$$
\epsilon_{r}^{*}=\frac{c^{2}}{\omega^{2}}\left[\left(\frac{2 \pi}{\lambda_{c}}\right)^{2}-\frac{1}{L^{2}}(\ln Z+2 \pi j n)^{2}\right] / \mu_{r}^{*}
$$

The correct value of $n$ is picked using the group delay comparison. At low frequencies the correct value is more easily identified since the roots are more widely spaced.

Equation (39) has an infinite number of roots for magnetic materials, since the logarithm of a complex number is multi-valued. In order to pick out the correct root, we have to compare the measured group delay to the calculated group delay. The 
calculated group delay is related to the change of the wave number $k$ with respect to the angular frequency

$$
\begin{gathered}
\tau_{\text {calc.group }}=-L \frac{d}{d f} \sqrt{\frac{\epsilon_{r}^{*} \mu_{r}^{*} f^{2}}{c^{2}}-\frac{1}{\lambda_{c}^{2}}} \\
=-\frac{1}{c^{2}} \frac{f \epsilon_{r}^{*} \mu_{r}^{*}+f^{2} \frac{1}{2} \frac{d\left(\epsilon_{r}^{*} \mu_{r}^{*}\right)}{d f}}{\sqrt{\frac{\epsilon_{r}^{*} \mu_{r}^{*} f^{2}}{c^{2}}-\frac{1}{\lambda_{c}^{2}}}} L .
\end{gathered}
$$

The measured group delay is

$$
\tau_{\text {meas.group }}=\frac{1}{2 \pi} \frac{d \phi}{d f}
$$

where $\phi$ is the phase of $Z_{1}$. To determine the correct root, the calculated group delays are found from eq (41) for various values of $n$ in the logarithm term, where $\ln Z=\ln |Z|+j(\theta+2 \pi n)$, and $n=0, \pm 1, \pm 2, \ldots$. The calculated and measured group delays are compared to yield the correct value of $n$. Where there is no loss in the sample under test, eq (39) is divergent at integral multiples of one-half wavelength in the sample. This occurs because for small $\left|S_{11}\right|$, the phase of $S_{11}$ cannot be accurately measured. Also in this limit, both scattering equations reduce to the relation $Z^{2} \rightarrow 1$, which is a relation for the phase velocity only and therefore solutions for $\epsilon_{r}^{*}$ and $\mu_{r}^{*}$ are not separable. This singular behavior can be minimized in cases where permeability is known a priori.

For a short-circuited line the equation, for the reflection coefficient is

$$
\Gamma=\frac{\tanh \gamma L+\beta \tanh \gamma_{0} \Delta L-\beta\left(1+\beta \tanh \gamma L \tanh \gamma_{0} \Delta L\right)}{\tanh \gamma L+\beta \tanh \gamma_{0} \Delta L+\beta\left(1+\beta \tanh \gamma L \tanh \gamma_{0} \Delta L\right)},
$$

where $\Delta L$ is the distance of the sample from the short circuit, and

$$
\beta=\frac{\gamma \mu_{o}}{\gamma_{o} \mu}
$$


A list of cutoff frequencies and operating frequencies for various waveguide sizes is given in tables 13 and 14. Coaxial line has the distinct advantage of having no cutoff frequency; however, coaxial line becomes multi-moded above a certain frequency. These multi-modes are due to bead resonance, which couples in the $T E_{11}$ and higher modes. The approximate upper frequency limit due to bead resonance is given in table 15. At very low frequencies $(\leq 100 \mathrm{kHz})$, skin-depth effects change the impedance due to changes in the inductance.

Table 13. Cutoff frequencies for rectangular waveguides.

\begin{tabular}{c|cc}
\hline EIA WR & Band & Cutoff frequency $(\mathrm{GHz})$ \\
\hline 650 & L & 0.908 \\
430 & W & 1.372 \\
284 & S & 2.078 \\
187 & C & 3.152 \\
90 & X & 6.557 \\
42 & K & 14.047 \\
22 & Q & 26.342 \\
\hline
\end{tabular}

Table 14. Rectangular waveguide dimensions $(a),(b)$ and operating frequencies in air.

\begin{tabular}{c|cccc}
\hline EIA WR & Band & $a(\mathrm{~cm})$ & $b(\mathrm{~cm})$ & $T E_{10}$ Operating Frequency $(\mathrm{GHz})$ \\
\hline 650 & $\mathrm{~L}$ & 16.510 & 8.255 & $1.12-1.7$ \\
430 & $\mathrm{~W}$ & 10.922 & 5.461 & $1.7-2.6$ \\
284 & $\mathrm{~S}$ & 7.710 & 3.403 & $2.6-3.95$ \\
187 & $\mathrm{C}$ & 4.754 & 2.214 & $3.95-5.85$ \\
90 & $\mathrm{X}$ & 2.286 & 1.016 & $8.2-12.4$ \\
42 & $\mathrm{~K}$ & 1.067 & 0.432 & $18-26.5$ \\
22 & $\mathrm{Q}$ & 0.569 & 0.284 & $33-50$ \\
\hline
\end{tabular}


Table 15. Air-filled coaxial cable operating frequencies.

\begin{tabular}{cc}
\hline Coaxial cable dimensions $(\mathrm{mm})$ & Useful operating frequency $(\mathrm{GHz})$ \\
\hline 3.5 & $0.001-34.5$ \\
7.0 & $0.001-18.2$ \\
14.0 & $0.001-08.6$ \\
\hline
\end{tabular}




\subsubsection{Open-Ended coaxial lines and waveguides}

- Open-Ended Coaxial Probes

Open-ended coaxial lines and waveguides $[80,81]$ have been used for years as nondestructive testing tools. In the open-ended coaxial or waveguide measurement, the probe is pressed against a specimen and the reflection coefficient is measured and used to determine the permittivity. Coaxial probes (see figure 18) have also been used for elevated temperature measurements of solids and liquids [82]. The technique has been popular and studied extensively. Although nondestructive, the method has definite limitations. At low frequencies there is little field interaction with the material. Since the coaxial probe has electric field components in both axial and radial directions, the measurement contains components of both the axial and radial permittivities. The method is sensitive to air gaps since the probe has both $E_{z}$ and $E_{\rho}$ electric field components. The open-ended waveguide technique has the advantage of a tangential electric field at the probe end, thus minimizing the effect of an air-gap. In process control, for example in thickness testing, a noncontacting probe may be required [82-89]. For this reason it is important to have a model of a coaxial probe which includes lift-off [86], or inclusion of an air gap between sample and probe, as shown in figure 18. The coaxial probe operates at a frequency where only the fundamental TEM mode propagates in the coaxial line. Evanescent $\mathrm{TM}_{o n}$ modes are also assumed to exist in the coaxial line near the probe end. In the coaxial line and the material under test, the magnetic field is assumed to be azimuthally symmetric. Therefore, only the $H_{\phi}$ component need be calculated. Two coaxial probes can be used to form a two-port test device. In this type 


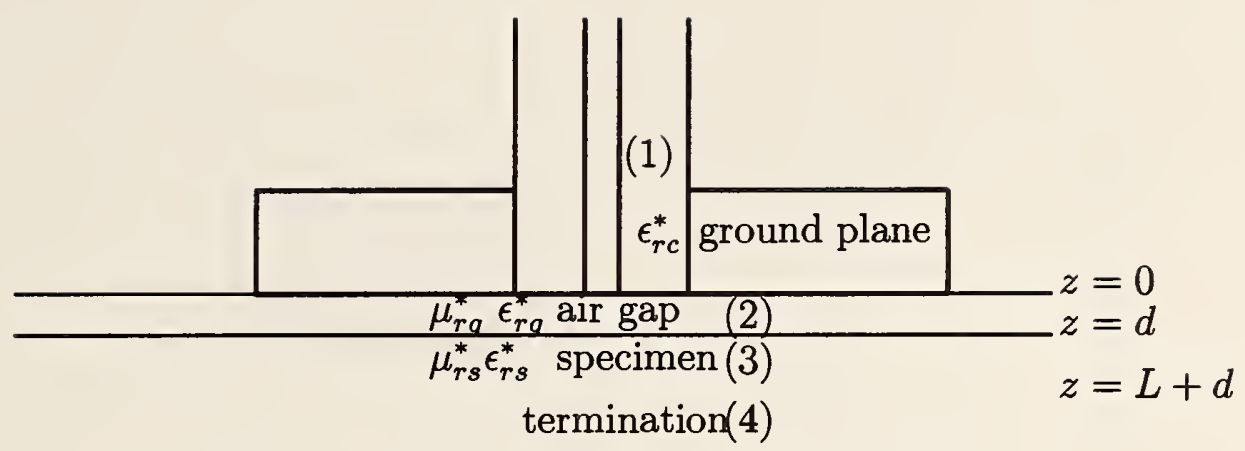

Figure 18. The open-ended coaxial probe over a specimen with an air gap between specimen and probe.

of fixture, a specimen is inserted between the probes and both reflection and transmission data is obtained and inverted for permittivity. The full-mode model is given in reference [90]. The analytical solution follows the same line of reasoning used in the full-mode single coaxial-probe problem. Experimental results indicate that the coaxial probe is useful for broadband material measurements on thin materials as shown in figures 19 and 20. Uncertainties in open-ended coaxial measurements include dimensional and scattering parameter measurement uncertainties.

In figures 19 and 20, coaxial probe measurements on FR-4 are compared to results on the reentrant cavity and full-sheet resonance methods. We see that the coaxial probe measurement has higher uncertainty, but covers a larger frequency band.

The coaxial probe has the advantage of nondestructive testing capability, but the uncertainties are larger than for resonant techniques.

- Short-circuited Open-ended Probes

Another method that has been used over the years is a variation of the coaxial probe, where the sample is positioned at the end of the inner conductor as 


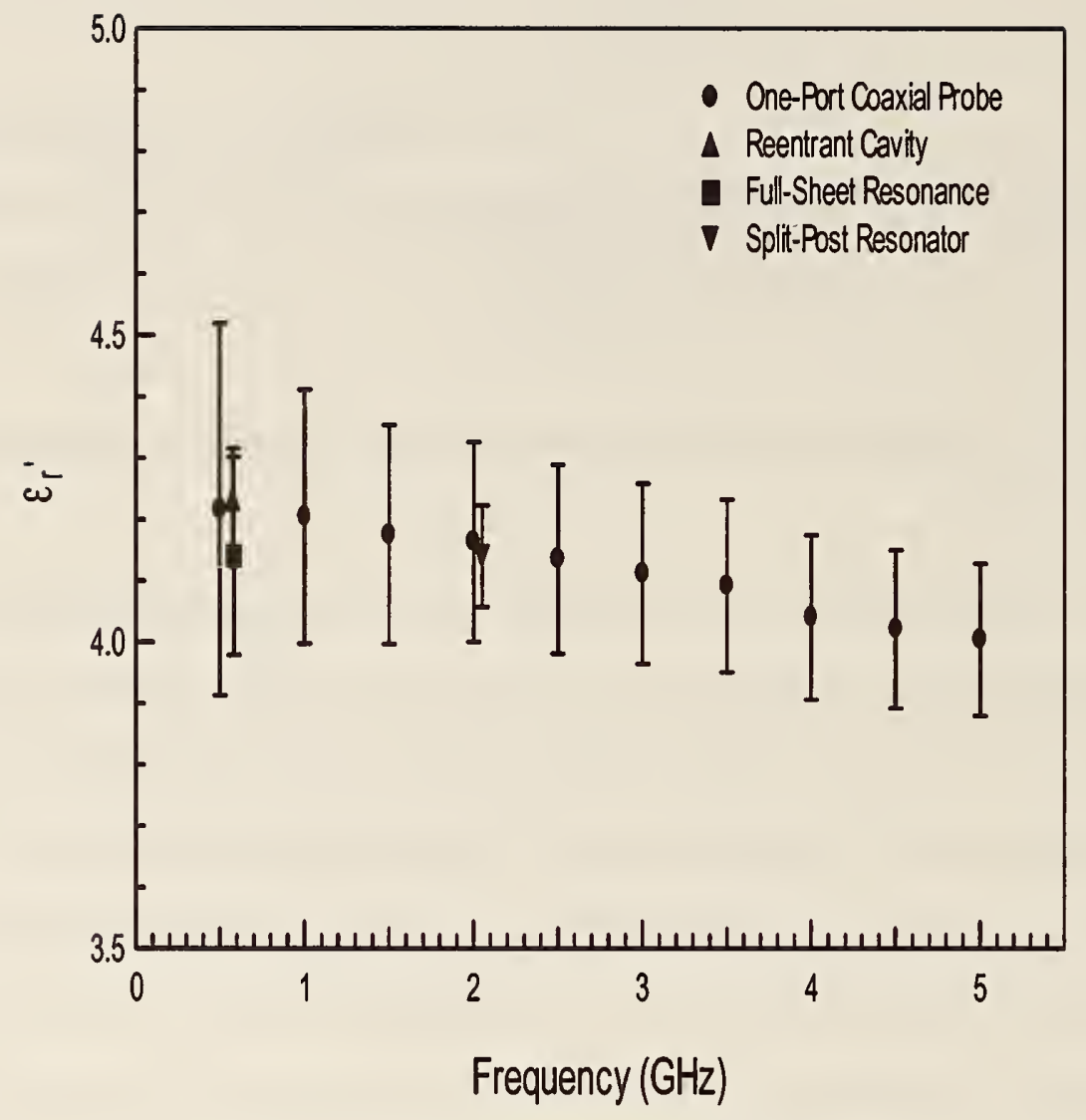

Figure 19. $\epsilon_{r}^{\prime}$ from measurements of FR-4 material using a coaxial probe compared to measurements made by reentrant cavity, split-post resonator, and full-sheet resonance techniques.

shown (a) in figure 21 [91-93]. A full-mode model of configuration (b) in figure 21 has been published [94]. Since the sample rests on a metal surface, the method has limited loss resolution.

- Shielded Open-Circuited Holders

A useful measurement method for liquids, semisolids, and solids over broad frequencies is the shielded open-circuited holder as shown in figure 22. The shield extends beyond the inner-conductor termination. The open-circuit posi- 


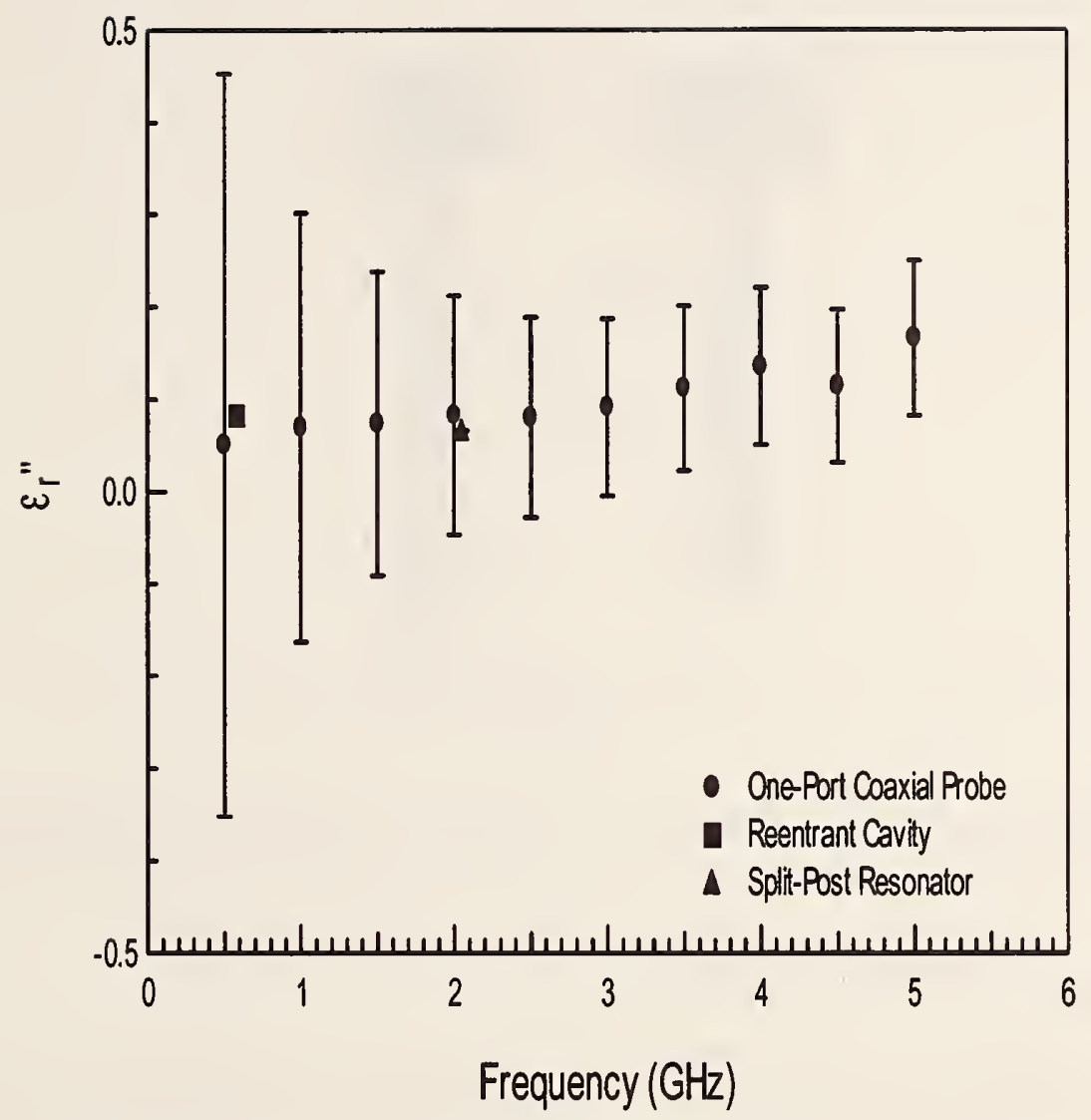

Figure 20. $\epsilon_{r}^{\prime \prime}$ from measurements of a FR-4 material using a coaxial probe compared to measurements made by reentrant cavity, split-post resonator, and full-sheet resonance techniques.

tion is below the fill level of the liquid or powder. The frequency of operation is assumed to be such that the modes in the shield region are evanescent. The dominant TEM mode is assumed to be incident upon the open circuit where a reflected $T E M$ wave is formed together with evanescent $T M_{0 n}$ modes. No energy is lost to the evanescent modes unless the lines are lossy or a lossy material is in the coaxial line or shield. 


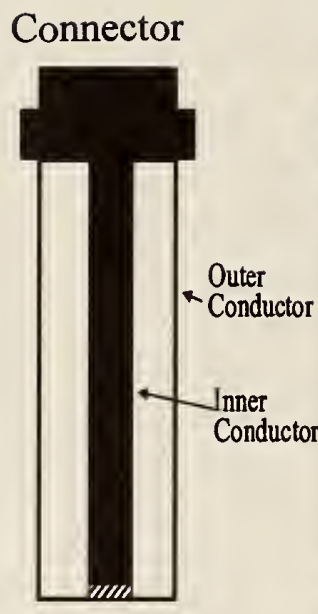

(a)

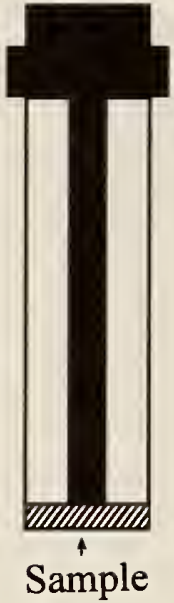

(b)

Figure 21. Open-ended sample method. (a) depicts the sample only under the inner post, and (b) the sample extends across the entire diameter of the coaxial line.

\subsection{Capacitive Techniques}

Capacitance techniques are important at frequencies in the range of dc to $100 \mathrm{MHz}$ [95-99]. In these techniques, the electric fields are nearly normal to the sample plane. The difficulties with these measurements pertain to air-gap depolarization, electrode polarization, and minimizing the effects of the fringing field. An air gap at the conductor/metal interface will yield a low value for the real part of the permittivity. The fringing field is usually partially eliminated by measuring the capacitance with and without a specimen and subtracting the results. Guards can reduce the effect of fringing fields, as shown in figure 23, if the guard and unguarded electrodes extend beyond the guard gap by a distance of at least twice the sample thickness (see ASTM method D-150). In a guarded capacitor the permittivity is

$$
\epsilon_{r}^{*}=\frac{C_{s}-j G_{s} / \omega}{C_{a i r}-j G_{a i r} / \omega},
$$




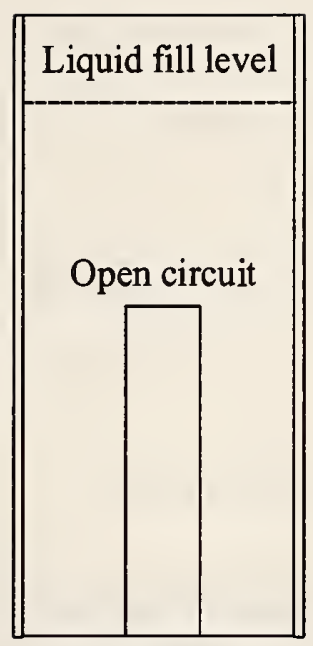

Connector

Figure 22. The shielded open-circuited holder.

where $C_{a i r}, G_{a i r}$ and $C_{s}, G_{s}$ are the capacitances and conductances with holder filled with air and specimen, respectively. At very low frequencies, for conducting materials, electrode polarization causes an anomalously high value for $\epsilon_{r}^{\prime}$. This is due to an extra capacitance that forms a double layer between the sample and electrode.

Two-fluid capacitor measurements are frequently used on PWB materials [100]. The technique has the advantage that the material thickness does not enter the calculation. The capacitance is measured in air and then in a fluid, both with and without the specimen. The equations are then solved for the permittivity. The fluids used in the method, such as n-heptane, must have very low loss and be well characterized. 


\section{Capacitor measurement}

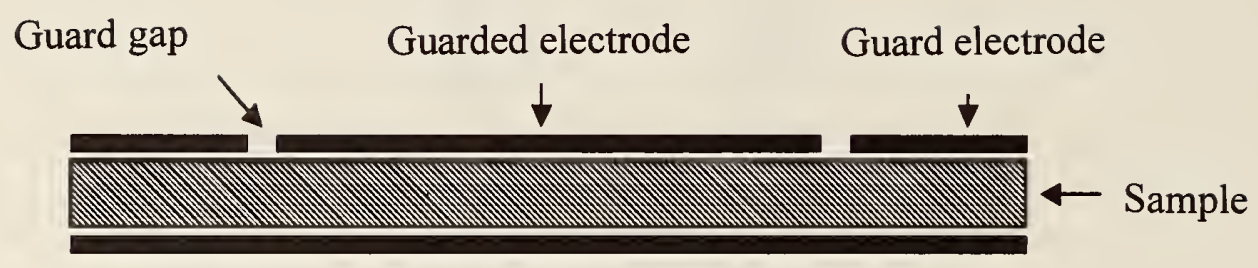

Unguarded Electrode

Figure 23. A sample in a capacitor with electrode guards.

\section{Dielectric Measurement and Metallic Loss Characterization Using Patterned Fixtures}

\subsection{Introduction to Metalized Structures}

Many transmission lines used in microelectronics have conductors deposited directly on a substrate. Examples of these include stripline, microstrip, coplanar waveguide, parallel plate, and coplanar strip. In the process of depositing metal films on the substrates, some metallic particles may penetrate into the substrate surface, causing a boundary-layer effect. This will affect both $\epsilon_{r}^{\prime}$ and $\tan \delta$. Also, since the metal films are very thin, the fields can penetrate through the film and cause additional loss as the wave travels through the dielectric. Therefore, in these types of structures it is important to characterize the system as a whole and attempt to deconvolve the 
metal from the dielectric losses. This becomes more important at higher frequencies where the dielectric and metal losses increase as approximately $\sqrt{\omega}$. In this section we introduce various measurement structures containing metalization and indicate how to characterize both the permittivity and metal losses. The metal losses in a resonant structure are related to the losses in the dielectric in terms of the quality factor of the dielectric, $Q_{d}$ and conductor, $Q_{c}$ as

$$
\frac{1}{Q}=\frac{1}{Q_{d}}+\frac{1}{Q_{c}}
$$

Calibration of patterned structures is complicated because the impedance is not always known and in addition usually is complex. Reference-plane calibration for patterned structures can be accomplished by transmission-reflection-load (TRL) algorithms (see Marks $[101,102]$ ). In these methods, standard transmission lines of varying lengths, short circuits, and open circuits are patterned onto well-characterized substrates.

\subsection{Conductors on Printed Wiring Boards}

The conductors on PWB can be separated into signal or ground/power conductors. These in turn can be separated by behavior as either lumped (resistance, inductance, capacitance) or distributed (transmission-line) elements. The differentiation between distributed and lumped is somewhat arbitrary and is based on the frequency at which the conductor's behavior is expected to change. The wavelength $\lambda$ is used to differentiate between lumped and distributed behavior as shown in figure 24. Each conductor has an electrical length that can be given in terms of the wavelength(s) of the signal(s) propagating in the conductor. The wavelength for a given frequency depends on the effective permittivity of the conductor environment. If the conductor's length is much greater than the wavelength $\left(L_{c}>>n \lambda\right)$ then the conductor's 


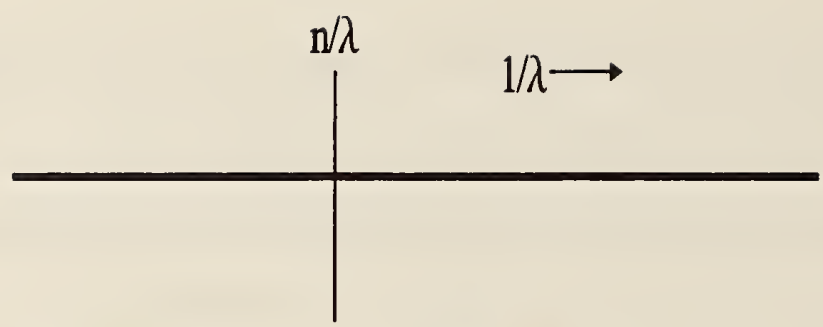

Lumped Distributed

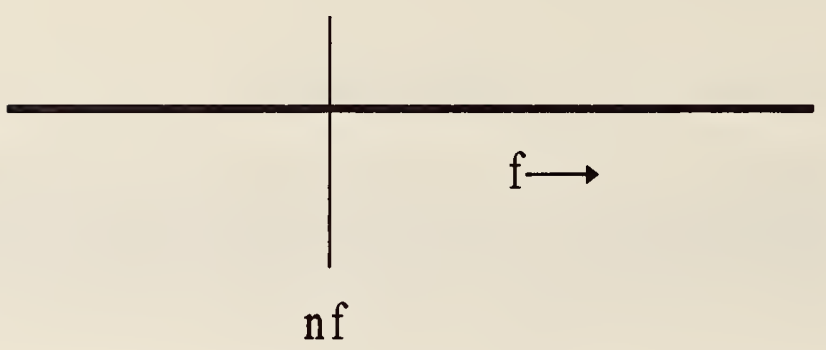

Figure 24. Lumped versus distributed parameters in a transmission line. For distributed circuits $L_{c}>>n \lambda$.

behavior is that of a transmission line. If the conductor length is much less than a wavelength $\left(L_{c}<<n \lambda\right)$ then the conductor behavior is that of a lumped element. Typically, the integer $n$ is between 3 to 10 .

Power and ground conductors are typically treated as lumped elements because of their size and configuration. Signal conductors, however, are either lumped or distributed, as defined above. If the signal conductor behaves as a lumped element, the relevant circuit parameters are inductance $(L)$, capacitance $(C)$, and resistance $(R)$. Conductance $G$ is related to dielectric losses. Conductors behaving as transmission lines are discussed in the next section. 


\subsection{Transmission-Line Parameters for Circuit-Board Applications}

Important parameters for transmission lines in PWB and LTCC applications are characteristic impedance, $Z_{c}$, and propagation constant $\gamma$. Knowledge of the characteristic impedance is important for transfer of power between circuits and circuit elements, reducing aberrant triggering caused by reflections, and maintaining signal integrity. The characteristic impedance is given by $[11,103]$

$$
Z_{C}=\sqrt{\frac{R(\omega)+j \omega L(\omega)}{G(\omega)+j \omega C(\omega)}},
$$

where $G$ is the dielectric conductance, $\omega=2 \pi f$, and $L$ and $R$ are the distributed inductance and resistance which may be frequency dependent. The capacitance $\mathrm{C}$ for low-loss dielectrics depends very weakly on frequency. The reason $L$ is a function of frequency is that it can be expressed as $L=L_{i n t}+L_{\text {ext }}$, where $L_{i n t}$ is the internal self inductance of the conductors and $L_{e x t}$ is the external inductance between the conductors. $R$ and $L_{\text {int }}$ depend on the skin depth. The skin depth, $\delta$, is related to the conductivity $\sigma$ by

$$
\delta=\frac{1}{\sqrt{\pi f \mu_{0} \mu_{r}^{\prime} \sigma}}
$$

where $\sigma$ is the dc conductivity. Equation (48) implies that the fields at lower frequencies penetrate further into materials than fields at higher frequencies. Very thin conductors allow the fields to penetrate appreciably through the metal. This increases resistance because the field exists in both the metal and the dielectric. As a consequence of the skin depth, the internal inductance decreases with increasing frequency, whereas $R$ increases with frequency. The resistance is given by

$$
R=\frac{1}{\sigma} \frac{L_{e}}{A_{e}}
$$


Table 16. Conductivities of metals.

\begin{tabular}{ccc}
\hline Material & $\begin{array}{c}\text { Conductivity, } \sigma(\mathrm{S} / \mathrm{m}) \\
/\left(10^{7} \mathrm{~S} / \mathrm{m}\right)\end{array}$ & $\begin{array}{c}\text { Skin depth } \delta(\mathrm{m}) \text { at }(10 \mathrm{GHz}) \\
/\left(10^{-7} \mathrm{~m}\right)\end{array}$ \\
\hline $\mathrm{Cu}$ & 5.80 & 6.60 \\
$\mathrm{Al}$ & 3.72 & 8.26 \\
$\mathrm{Ag}$ & 6.17 & 6.42 \\
$\mathrm{Au}$ & 4.10 & 7.85 \\
$\mathrm{In}$ & 0.87 & 17.0 \\
$70-30$ brass & 1.57 & 12.70 \\
Typical solder & 0.71 & 19.0 \\
\hline
\end{tabular}

where $L_{e}, A_{e}$ are the length, and effective area of where the current passes through the conductor. Using eq (48) in eq (49), we see that resistance increases with frequency.

Electromagnetic losses due to metals increase as frequencies increase due to the surface resistivity defined in ohms/square as

$$
R_{s}=\frac{1}{\delta \sigma}=\sqrt{\frac{\pi f \mu_{0} \mu_{r}^{\prime}}{\sigma}} .
$$

Total conductor loss is a function of conductivity of the metal and of the thickness, width, surface roughness, and boundary layer between metal and substrate. Clad materials can have appreciable surface roughness, in order to promote adhesion. The copper foil that is applied to the PWB material has a roughened surface. Surface roughness affects the measured capacitance, inductance, and resistance. The effect of surface roughness on the resistance is small at low frequencies. However, above $1 \mathrm{GHz}$, the resistance of rough copper increases noticeably over that of smooth copper $[2,31]$. Bulk electrical conductances of commonly used metals are given in table 16 [103].

The conductance $G$ of the transmission line depends on the dielectric substrate. At low gigahertz frequencies or below, these losses are smaller than conductor losses 
for PWB applications. However, the losses in the dielectric increase roughly in proportion to $\omega$, whereas conductor losses increase as $\sqrt{\omega}$. Therefore, at high frequencies, above approximately $10 \mathrm{GHz}$ to $30 \mathrm{GHz}$, the dielectric loss can exceed metal losses.

In an ideal lossless transmission line for typical wireless frequencies, we can approximate eq (47) with

$$
Z_{C(\text { ideal })} \approx \sqrt{\frac{L}{C}}
$$

Equation (51) shows that the characteristic impedance of the lossless transmission line is real and depends only on $L$ and $C . Z_{c}$ can be complex [101,104]. An approximation for the characteristic impedance at high frequencies can be obtained by a Taylor-series expansion in $R$. Assuming $G=0$,

$$
Z_{c} \approx \sqrt{\frac{L}{C}}\left[1-j \frac{R}{2 \omega L}+\frac{R^{2}}{8 \omega^{2} L^{2}}\right] .
$$

The propagation constant $\gamma$ is also important in transmission-line performance because it describes the attenuation and phase response of the line

$$
\gamma=\alpha+j \beta=\sqrt{(R(\omega)+j \omega L(\omega))(G+j \omega C)} \approx \frac{R}{2 Z_{0}}+j \omega \sqrt{L C}\left[1-\frac{R^{2}}{8 \omega^{2} L^{2}}\right],
$$

where $C, G, L, R$ are measured distributed circuit parameters per unit length. The last expression in eq (53) is a Taylor-series expansion of $\gamma$ with $G=0$ in powers of R. $\alpha$ and $\beta$ are attenuation and phase parameters. $\alpha$ includes loss due to conductor, dielectric, and radiation: $\alpha=\alpha_{c}+\alpha_{d}+\alpha_{r}$. Here $\alpha_{c}$ can be approximated by [11]

$$
\alpha_{c}=\frac{R(\omega)}{2 Z_{c(\text { ideal })}}
$$

and

$$
\alpha_{d}=\frac{1}{2} G Z_{c(\text { ideal })}=\frac{\omega}{2} \sqrt{\frac{\mu_{0} \mu_{r}^{\prime}}{\epsilon_{0} \epsilon_{r}^{\prime}}} \epsilon_{r}^{\prime \prime}=\frac{\omega}{2} \sqrt{\epsilon_{0} \epsilon_{r}^{\prime} \mu_{0} \mu_{r}^{\prime}} \tan \delta,
$$


where $\epsilon_{r}=\epsilon_{0}\left(\epsilon_{r}^{\prime}-j \epsilon_{r}^{\prime \prime}\right)$ is the permittivity of the substrate, $\epsilon_{0}$ is the permittivity of vacuum and $\tan \delta=\epsilon_{r}^{\prime \prime} / \epsilon_{r}^{\prime}$ is the loss tangent in the material. The loss tangent in terms of distributed circuit parameters and frequency, $f$, is $\tan \delta=G /(2 \pi f C)$.

The imaginary contribution to the propagation constant defines the phase of an electromagnetic wave. The phase velocity in a transmission line is given by $v=\omega / \beta$. For a uniform lossless line $v=1 / \sqrt{L C}$. For a lossy line

$$
v_{p}=\frac{1}{\sqrt{L C}} \frac{1}{1-\frac{R^{2}}{8 \omega^{2} L^{2}}} .
$$

Since the propagation time varies as a function, in a LTCC or PWB applications the signals with high harmonic content, such as pulsed waveforms, will exhibit dispersion in propagating along the line. Furthermore, the pulse will experience frequencydependent losses that will increase the dispersion in the signal.

\subsection{Conductor-Loss Measurement}

The insertion loss $\left(L_{I}\right)$ is commonly used to measure line loss [105]. The definition of insertion loss is

$$
L_{I}=10 \log \frac{P_{1}}{P_{2}}
$$

where $P_{1}$ is the power delivered to a load in the absence of the network and $P_{2}$ is the power delivered to the load with the network inserted in the system. Therefore, the insertion loss depends on both the impedance match between the load and the characteristics of the network. Dissipative losses can be separated from reflection power losses as [106]

$$
L_{I 1}=\frac{1}{L}\left[-10 \log \left(1-\left|S_{11}\right|^{2}\right)-10 \log \frac{\left|S_{21}\right|^{2}}{1-\left|S_{11}\right|^{2}}\right] .
$$

The attenuation $(\alpha)$ is assumed to be the insertion loss $\left(L_{I}\right)$ for a reflectionless system. Here $L_{I 1}$ denotes distributed insertion loss $(\mathrm{dB} / \mathrm{m})$. 
The main issue when measuring insertion loss is accounting for effects of reflections at connectors. Other methods that are commonly used include a measurement of two line lengths, $L_{1}$ and $L_{2}$,

$$
L_{I 2}=\frac{-20 \log \left|S_{21}\right|\left(L_{1}\right)+20 \log \left|S_{21}\right|\left(L_{2}\right)}{L_{1}-L_{2}}(\mathrm{~dB} / \mathrm{m}) .
$$

Here $L_{I 2}$ denotes distributed insertion loss $(\mathrm{dB} / \mathrm{m})$. Equation (59) is equivalent to eq (58) except it used two line lengths. This method has the advantage of subtracting out the effects of the calibration and sample holder mismatch, assuming it is the same for each measurement. Another method is based on the transmission coefficient [107]

$$
T=1+\Gamma=1+\frac{Z_{1}-Z_{0}}{Z_{1}+Z_{0}}
$$

where

$$
L_{I}=-20 \log |T|(\mathrm{dB})
$$

A measurement of attenuation from the propagation constant [102] using coplanar waveguide on gallium arsenide is given in figure 25. The measurements were calibrated with NIST-developed software called MULTICAL.

\subsection{Time-Domain PWB Measurement Methods}

Broadband methods using a time-domain reflectometry (TDR) technique have been developed to extract $\epsilon_{r}^{\prime}$ from PWB dielectrics $[7,10,44,108]$. Janezic compared timedomain versus frequency-domain measurement accuracy [44]. A technique that uses frequency averaging is given in reference [10]. In this method the average permittivity over the frequencies contained in the pulse is determined. The specimen used in this technique is a parallel-plate transmission line (PPTL) and is placed in the holder shown in figure 26. The permittivity of the dielectric can be found in two 


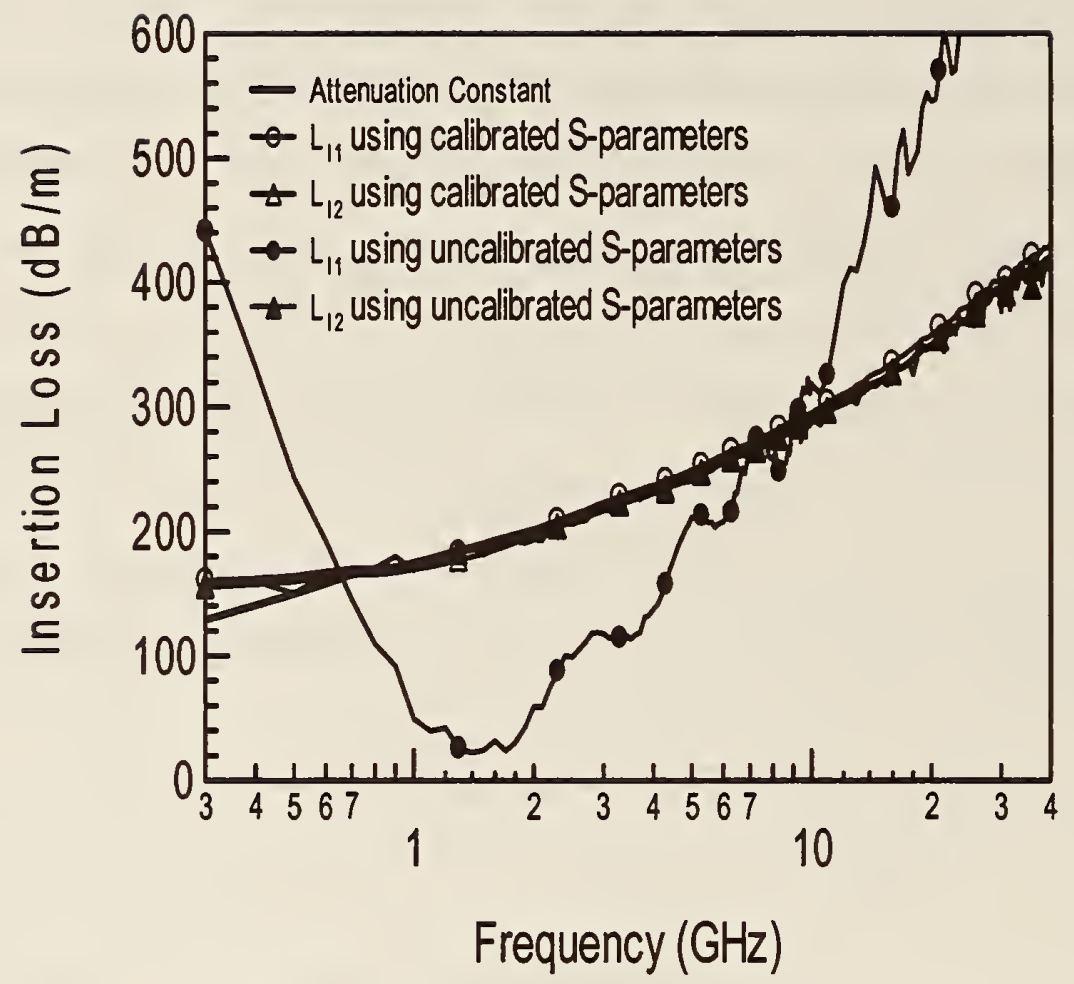

Figure 25. Insertion loss for coplanar waveguide on gallium arsenide using (58), (59), attenuation coefficient, and uncalibrated scattering parameters. Conductor width is $50 \mu \mathrm{m}$, conductor thickness is $3 \mu \mathrm{m}$.

ways. In the first way, several specimens are used each with a different line width. The relationship between the reciprocal of the measured impedance of the line versus line width is a straight line with a slope proportional to permittivity (this assumes a linear material); the intercept of the line provides information on the effects of the fringing field. In the second, the fringing field effects are approximated by adding a parallel line transmission line to the ideal PPTL model. However, this approximation does not work for large ratios of thickness to width. The measurements using this TDR method are in close agreement with those obtained from frequency-domain resonant methods $[80,10]$. A detailed uncertainty analysis is available in the refer- 


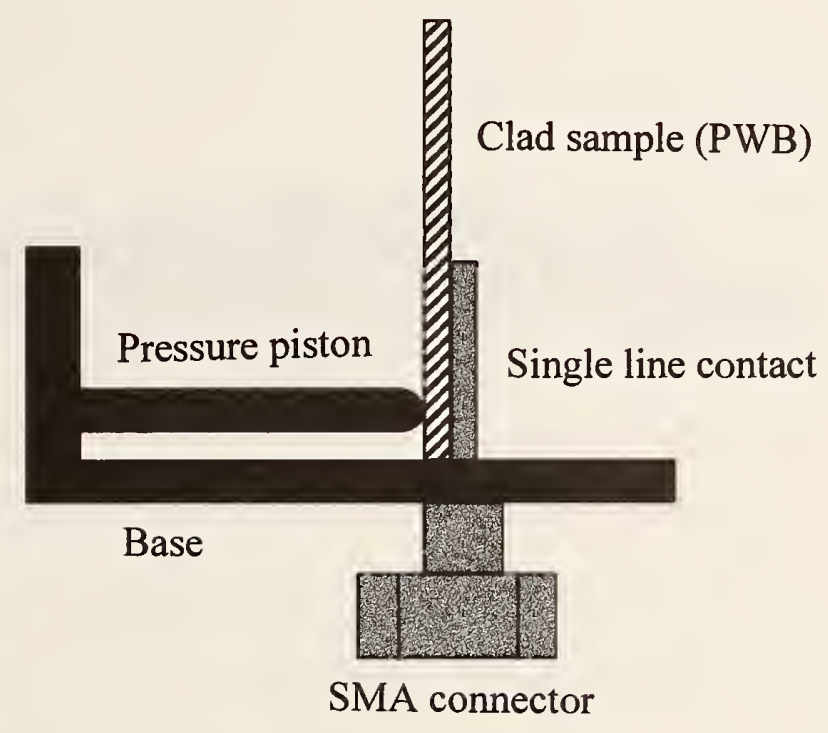

Figure 26. Sample for time-domain method.

ences[80]. The advantage of this method is that relatively inexpensive TDR systems and specimen holders can be used, and specimens fabricated with a router or shear. No photolithography or wet processing is required.

\subsection{Full-Sheet Resonance Method for Thin Sheets}

The full-sheet resonance technique is used to determine the permittivity of clad circuit and PWB materials (see figure 27) $[67,109,110]$. It is a nondestructive test and measures the bulk property of a substrate. The frequency of measurement is limited by the specimen dimensions since the method uses one-half wavelength 


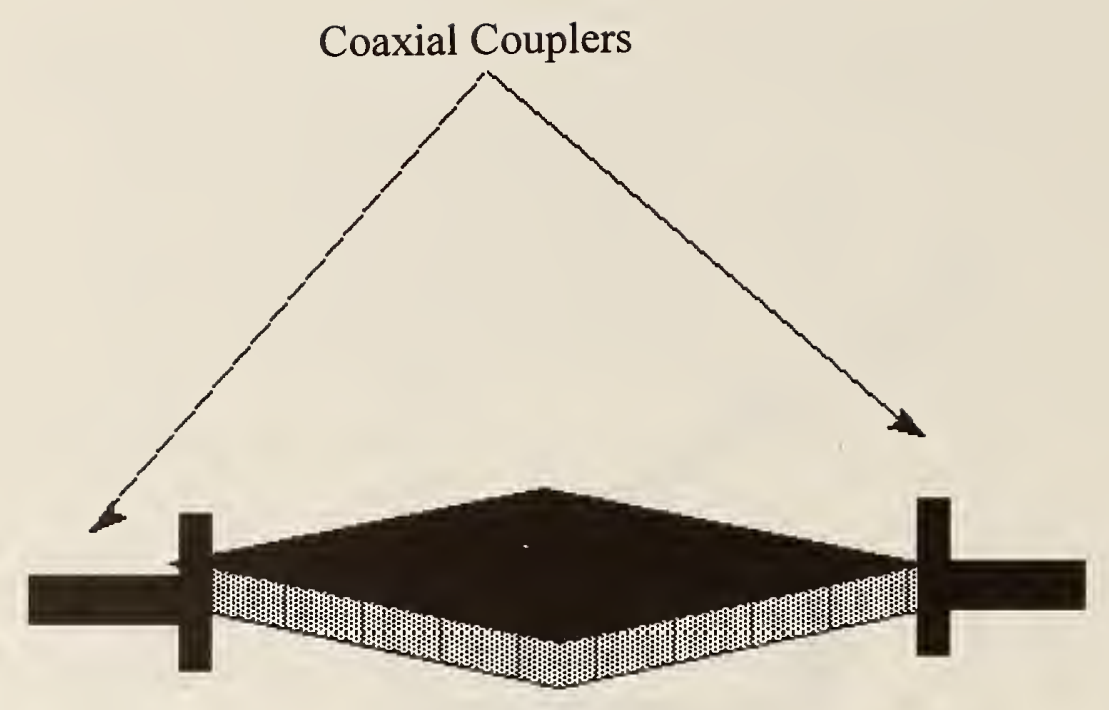

Figure 27. Full-sheet resonance technique.

resonances. The specimen is usually open at the sides, however some researchers measure with the sides closed by conductors. The open edges make the electrical length of the specimen slightly longer than the actual physical dimensions. The permittivity is measured by transmission and reflection coefficients of test signals introduced by coaxial feeds. This method causes the cladded structure to resonate at integral multiples of one-half wavelength of the wave in the specimen. Higher modes can also be used to extend the frequency coverage. The main difficulty in this technique is mode identification and degeneracy. The full-sheet resonance method has limited ability in determination of the loss factor since the surface area of the metal cladding is large relative to the volume of the specimen.

A set of measurements using the full-sheet resonance method (FSR) on a number of modes is shown in tables 17 and 18 and is compared to measurements with the reentrant cavity [109]. A circle fit procedure yields a more accurate quality factor and is summarized in [109]. 
The relative permittivity is obtained from the resonant frequencies of a cavity. The resonance condition for $\mathrm{TE}_{m n}$ modes is

$$
\epsilon_{r}^{\prime}=\left(\frac{c}{2 f_{m n}}\right)^{2}\left[\left(\frac{m}{a}\right)^{2}+\left(\frac{n}{b}\right)^{2}\right]
$$

where $c$ is the speed of light, $a$ and $b$ are plate dimensions, $m$ and $n$ are mode numbers, and $f_{m n}$ are the resonant frequencies. Note that the equation does not depend on specimen thickness. This lack of sensitivity to thickness has an advantage over other techniques.

The measured resonant frequencies calculated from eq (62) differ from the theoretical frequencies because of the open edges, conductivity of the plates, and radiation losses. The very low quality factors in this technique produce frequency-pulling (that is, lowering of the resonant frequency due to losses) that must be taken into account. Mode interference can also be a problem. Generally, better mode separation is possible if the specimen is rectangular rather than square.

Collin [111] derived an expression that accounts for frequency pulling due to finite wall conductivity

$$
f_{l}=f_{u l} \sqrt{1+\frac{1}{Q_{c}}+\frac{B_{R}}{G_{R} Q_{R}}},
$$

where $f_{l}$ and $f_{u l}$ refer respectively to loaded and unloaded resonant frequencies. $Q_{R}$ is the radiation quality factor, and $Y_{R}=G_{R}+j B_{R}$ is the open-side aperture admittance [109].

The quality factors $Q_{C}, Q_{R}$, and $Q_{e}$ of cavity, radiation, and coupling ports, are related to the loaded $Q_{L}$ by

$$
\frac{1}{Q_{L}}=\frac{1}{Q_{C}}+\frac{1}{Q_{R}}+\frac{1}{Q_{e}}=\frac{1+\kappa}{Q_{0}}
$$

where $\kappa$ is the cavity-coupling coefficient. 
Table 17. FSR on $0.38 \mathrm{~mm}$ thick PWB panel using various modes. The reentrant cavity result was $\epsilon_{r}^{\prime}=2.45,0.02$ at $440 \mathrm{MHz}$.

\begin{tabular}{c|cccc}
\hline TE Mode & $\mathrm{f}(\mathrm{MHz})$ & $\epsilon_{r}^{\prime}$ & Circle fit FSR & Uncertainty $(\mathrm{U}, k=2)$ \\
\hline 01 & 310 & 2.476 & 2.488 & \pm 0.007 \\
10 & 439 & 2.466 & 2.478 & \pm 0.009 \\
11 & 539 & 2.459 & 2.449 & \pm 0.017 \\
02 & 621 & 2.474 & 2.478 & \pm 0.011 \\
\hline
\end{tabular}

Table 18. FSR on $1.41 \mathrm{~mm}$ thick panel of FR4. The reentrant cavity result was $\epsilon_{r}^{\prime}=4.22, \pm 0.01$ at $568 \mathrm{MHz}$. Open-ended coaxial probe yielded $\epsilon_{r}^{\prime}=4.23 \pm 5 \%$ at $580 \mathrm{MHz}$.

\begin{tabular}{c|cccc}
\hline TE Mode & $\mathrm{f}(\mathrm{MHz})$ & $\epsilon_{r}^{\prime}$ & Circle fit FSR & Uncertainty $(\mathrm{U}, k=2)$ \\
\hline 01 & 234 & 4.059 & 4.184 & \pm 0.060 \\
10 & 333 & 4.092 & 4.158 & \pm 0.080 \\
11 & 405 & 4.001 & 4.185 & \pm 0.128 \\
02 & 473 & 4.084 & 4.118 & \pm 0.096 \\
12 & 578 & 4.019 & 4.140 & \pm 0.080 \\
\hline
\end{tabular}

Equation (62) can be corrected for the open sides of the specimen by use of the capacitance of the plates, $C$, and fringing capacitance, $C_{f}$ to give [109]

$$
\epsilon_{r}^{\prime}=\frac{C+C_{f}}{C+C_{f} / \epsilon_{r}^{\prime}}\left[\frac{c}{2 f_{m n}}\right]^{2}\left[\left(\frac{m}{a}\right)^{2}+\left(\frac{n}{b}\right)^{2}\right] .
$$

\subsection{Techniques for Transmission Lines on Substrates}

Transmission-line techniques are useful in PWB applications since the electric field orientation is roughly the same as in real applications. Various transmission-line techniques have been used for nondestructive determination of substrate properties $[27,76-78,112-115]$. These techniques can be broadly grouped under resonant or nonresonant techniques. The permittivity of a substrate or substrate-thin film composite can be obtained by forming either a stripline (see figure 32), microstrip 
(see figure 28), or coplanar waveguides (see figure 40), and then either resonating the system or looking at the combined transmission and reflection response.

In numerical modeling, an effective permittivity $\epsilon_{e f f}$ usually is defined that includes the effects of the fields in the air region surrounding the transmission line. As a result, the effective permittivity depends both on the fields in the specimen and the fringing fields. Theoretical models are usually available that extract the material's permittivity from the effective permittivity [116-118]. These models also estimate the characteristic impedance. In many applications, transmission lines have open surfaces. Open structures can radiate and have a complicated field structure $[3,116-130]$. Conductors may be etched away from wiring board cladding to form transmission lines, or alternatily, the conductor may be deposited on a substrate or thin film. Green tape LTCC materials are not usually clad, so conductors must be deposited on the material. PWB materials are thin and usually clad by copper, making measurements with closed waveguide or coaxial transmission lines difficult. Also, many substrates and PWB materials are laminated, so the component of permittivity normal to the sample face is different from the in-plane permittivity.

\subsubsection{Microstrip Resonator}

A microstrip consists of a strip and a ground plane separated by a dielectric. A drawing of a microstrip is given in figure 28. The quasi-TEM field in a microstrip exists in both the air region above the strip and in the dielectric substrate. Measurements can be made with the microstrip as a transmission line or resonator.

In a microstrip resonator, the effective permittivity can be estimated in terms of the length $L$, fringing-field correction $\Delta L$, frequency $f$, and resonance number $N$ 
as $[131]$

$$
\epsilon_{e f f}=\frac{N^{2} c^{2}}{4 f^{2}(L+\Delta L)^{2}}=\left(\frac{N c}{\lambda f}\right)^{2}
$$

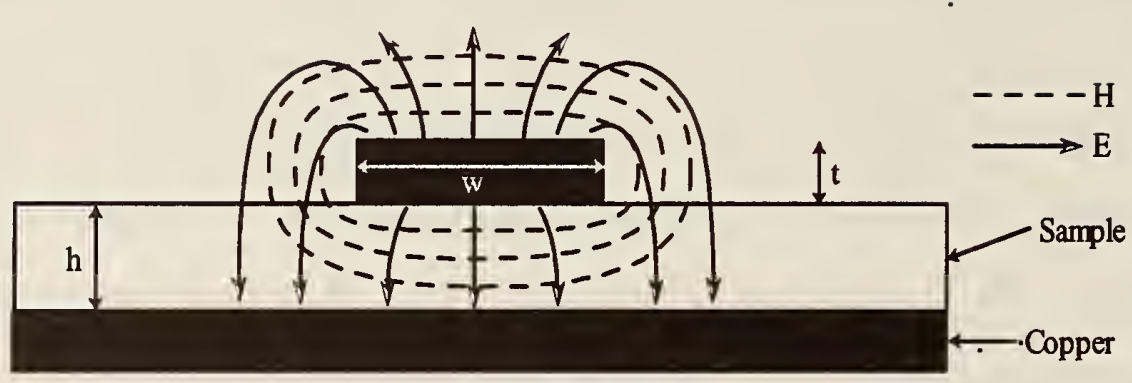

Figure 28. Cross section of a microstrip.

In a microstrip fixture with thin dielectrics or wide signal lines such that $w / h \geq 1$, where $w$ is the width, and $h$ is thickness of the substrate, $\epsilon_{\text {eff }}$ can be written as [131]

$$
\epsilon_{e f f}=\frac{\epsilon_{r}^{\prime}+1}{2}+\frac{\epsilon_{r}^{\prime}-1}{2 \sqrt{1+12 \frac{h}{w}}}
$$

Solving for $\epsilon_{r}^{\prime}$ in eq 66 yields

$$
\epsilon_{r}^{\prime}=\frac{2\left(\frac{N c}{\lambda f}\right)^{2}+\frac{1}{\sqrt{1+12 \frac{h}{w}}}-1}{1+\frac{1}{\sqrt{1+12 \frac{h}{w}}}}
$$

When $w / h \leq 1$

$$
\epsilon_{e f f}=\frac{\epsilon_{r}^{\prime}+1}{2}+\frac{\epsilon_{r}^{\prime}-1}{2}\left[\frac{1}{\sqrt{1+12 \frac{h}{w}}}+0.04\left(1-\frac{w}{h}\right)^{2}\right] .
$$

Solving for $\epsilon_{r}^{\prime}$ gives

$$
\epsilon_{r}^{\prime}=\frac{2\left(\frac{N c}{\lambda f}\right)^{2}-1+\left[\frac{1}{\sqrt{1+12 \frac{h}{w}}}+0.04\left(1-\frac{w}{h}\right)^{2}\right]}{1+\left[\frac{1}{\sqrt{1+12 \frac{h}{w}}}+0.04\left(1-\frac{w}{h}\right)^{2}\right]} .
$$

The attenuation constant in a microstrip has been calculated [132]. However, if the ratio $t / \delta$ (where $\delta$ is the relevant skin depth and $t$ is the thickness of the conductor) is small or close to unity $[133,134]$, Wheeler's rule breaks down and gives 
unreliable results. The failure of analytical methods for thin conductors can be a serious limitation for the design and modeling of planar structures. Recently, Holloway presented loss expressions that are valid for arbitrary conductor thickness [133-136]. These expressions include skin effects and field penetration through conductors. In figures 29 through 31 attenuation in microstrip as a function of various parameters is displayed.

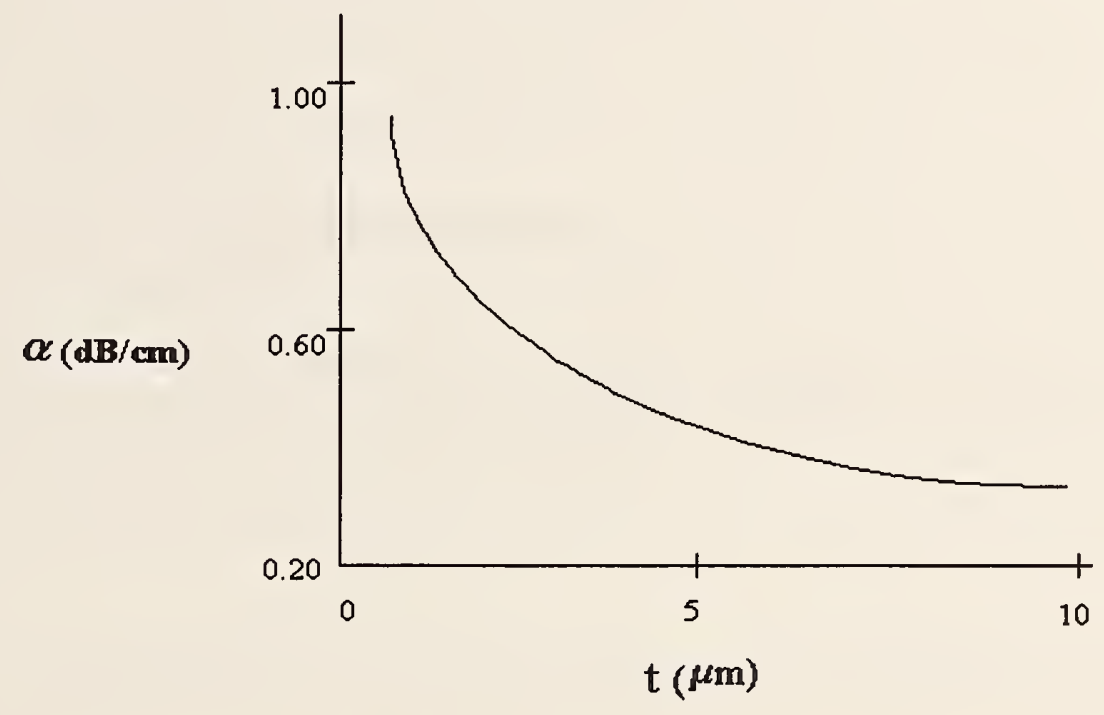

Figure 29. Strip conductor loss as a function of strip thickness for a microstrip line at $10 \mathrm{GHz}$, with geometries having $W=30 \mu \mathrm{m}, H=200 \mu \mathrm{m}, \epsilon_{r}^{\prime}=12.9, \sigma=$ $3.33 \times 10^{7} \mathrm{~S} / \mathrm{m}$. 


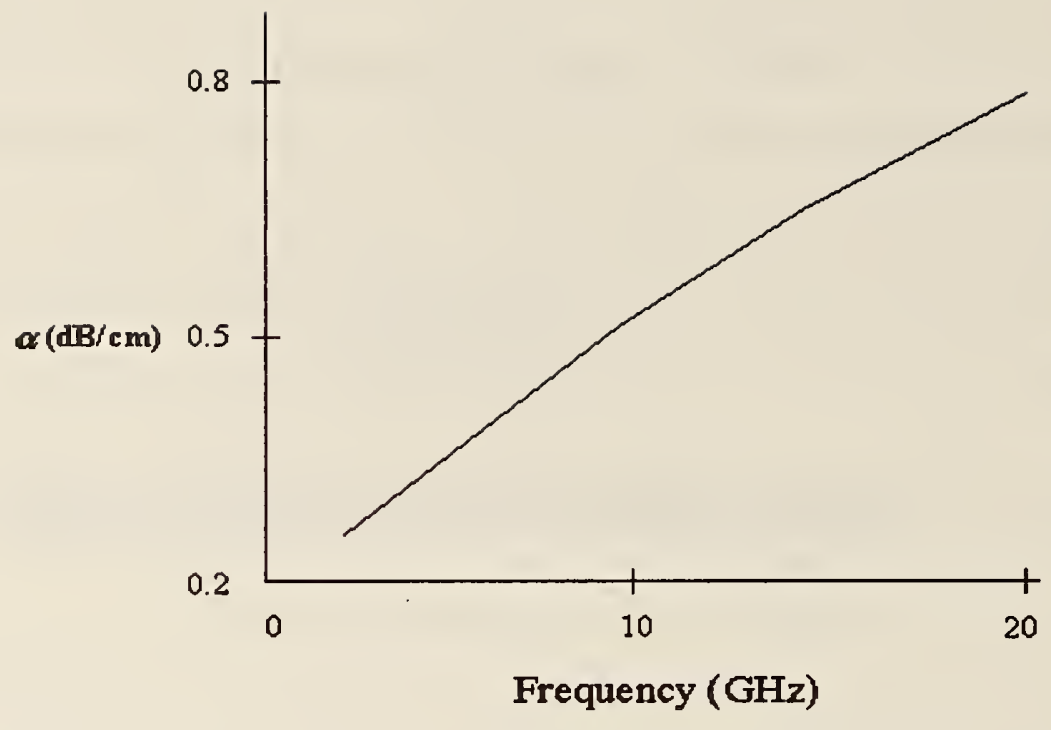

Figure 30. Strip conductor loss as a function of frequency for a microstrip line with geometries having $W=30 \mu \mathrm{m}, H=300 \mu \mathrm{m}, t=6 \mu \mathrm{m}, \epsilon_{r}^{\prime}=12.9, \sigma=1.77 \times 10^{7} \mathrm{~S} / \mathrm{m}$.

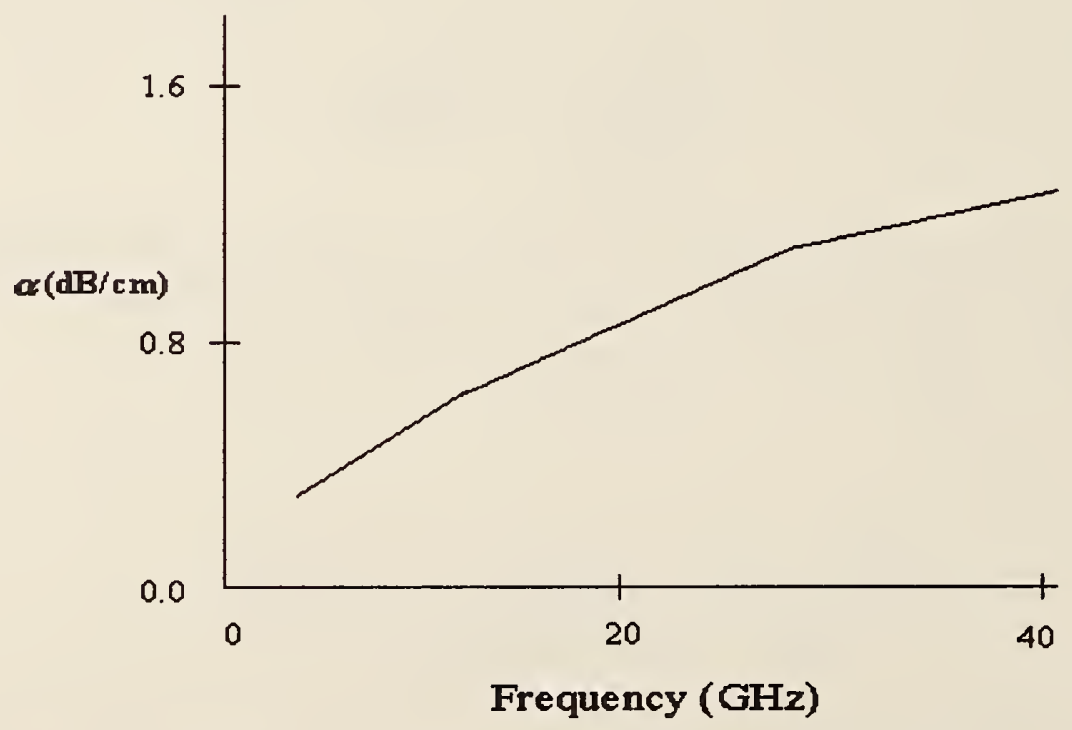

Figure 31. Strip conductor loss as a function of frequency for a microstrip line with geometries having $W=20 \mu \mathrm{m}, H=100 \mu \mathrm{m}, t=3 \mu \mathrm{m}, \epsilon_{r}^{\prime}=12.9, \sigma=4.1 \times 10^{7} \mathrm{~S} / \mathrm{m}$. 
Table 19. Values of the stopping distance for $90^{\circ}$ and $45^{\circ}$ edges.

\begin{tabular}{|c|c|c|c|c|c|c|c|c|c|c|c|}
\hline \multirow{2}{*}{$\frac{t}{2 \delta}$} & \multicolumn{2}{|c|}{$\frac{t}{\Delta}$} & \multirow{2}{*}{$\frac{t}{2 \delta}$} & & \multirow{2}{*}{$\frac{t}{2 \delta}$} & \multicolumn{2}{|c|}{$\frac{t}{\Delta}$} & \multirow{2}{*}{$\frac{t}{2 \delta}$} & \multicolumn{2}{|c|}{$\frac{t}{\Delta}$} \\
\hline & $90^{\circ}$ & $45^{\circ}$ & & $90^{\circ}$ & $45^{\circ}$ & & $90^{\circ}$ & $45^{\circ}$ & & $90^{\circ}$ & $45^{\circ}$ \\
\hline 0.03 & 9.18 & 6.57 & 0.87 & 138.42 & 115.75 & 1.87 & 266.73 & 306.15 & 5.0 & 174.33 & 327.01 \\
\hline 0.04 & 9.18 & 6.57 & 0.94 & 169.39 & 144.36 & 2.00 & 244.95 & 288.35 & 6.0 & 185.89 & 336.93 \\
\hline 0.05 & 9.19 & 6.57 & 1.0 & 200.50 & 173.95 & 2.18 & 221.57 & 271.18 & 7.0 & 193.43 & 357.86 \\
\hline 0.06 & 9.19 & 6.58 & 1.07 & 235.98 & 209.06 & 2.29 & 210.25 & 265.33 & 8.0 & 195.96 & 383.06 \\
\hline 0.10 & 9.25 & 6.62 & 1.12 & 258.21 & 232.17 & 2.51 & 200.43 & 264.15 & 9.0 & 196.58 & 410.96 \\
\hline 0.14 & 9.45 & 6.77 & 1.15 & 270.22 & 245.19 & 2.76 & 189.28 & 274.11 & 9.49 & 196.94 & 426.04 \\
\hline 0.25 & 11.76 & 8.43 & 1.22 & 299.73 & 279.65 & 3.00 & 178.57 & 288.74 & 10.49 & 198.36 & 461.40 \\
\hline 0.50 & 33.97 & 26.72 & 1.32 & 324.12 & 314.30 & 3.55 & 170.73 & 317.89 & 12.25 & 203.04 & 543.07 \\
\hline 0.64 & 61.90 & 49.41 & 1.50 & 329.88 & 342.89 & 4.00 & 168.50 & 327.08 & 13.0 & 205.58 & 587.90 \\
\hline 0.71 & 81.32 & 65.51 & 1.63 & 312.74 & 339.00 & 4.53 & 171.59 & 327.42 & 14.0 & 209.24 & 659.63 \\
\hline 0.79 & 108.83 & 89.23 & 1.73 & 293.30 & 326.77 & 4.74 & 172.81 & 326.99 & 16.0 & 217.25 & 862.14 \\
\hline
\end{tabular}

For a microstrip line, the attenuation constant associated with the strip conductor is expressed as

$$
\alpha_{\text {strip }}=\frac{R_{s m}}{Z_{0}} \frac{\ln \left(\frac{w}{t} \frac{t}{\Delta}-1\right)}{2 \pi^{2} w},
$$

where $w$ is the strip width, $t$ is the strip thickness, and $\Delta$ is the stopping point for the integrations, as defined in [133] and given in table 19 for normal conductors.

Reference [135] gives values for $\Delta$ for superconductors. $R_{s m}$ is the modified Horton impedance boundary condition as discussed in [133] and given by

$$
R_{s m}=\omega \mu_{c} t \operatorname{Im}\left(\frac{\cot \left(k_{c} t\right)+\csc \left(k_{c} t\right)}{k_{c} t}\right)
$$

where $k_{c}$ is the wavenumber in the conductor. $Z_{o}$ is the characteristic impedance 
given by [137]

$$
Z_{o}=\frac{\eta_{o}}{4 \pi} \sqrt{\frac{2}{\epsilon_{r}+1}} \ln \left\{1+\frac{32 h}{w}\left[\frac{h}{w} r_{\epsilon}+\sqrt{\left(\frac{h r_{\epsilon}}{w}\right)^{2}+\left(\frac{\pi\left(\epsilon_{r}+1\right)}{16 \epsilon_{r}}\right)^{2}}\right]\right\},
$$

where $h$ is the height of the substrate, $\eta_{0}$ is the impedance of free space and

$$
r_{\epsilon}=\sqrt{0.4052+\frac{0.5160}{\epsilon_{r}}+\frac{0.0788}{\epsilon_{r}}}
$$

The total conductor loss has contributions from both the strip conductor and the ground plane. For narrow microstrip, the ground plane loss is a small part of the total loss, whereas for wide microstrip, the ground plane loss becomes more important, see [133] and [136]. In fact, for $w / h>>1$, the ground plane loss nearly equals the strip loss. The ground plane loss can be obtained from [138]

$$
\alpha_{g r}=\frac{R_{s}}{2 Z_{o} I^{2}} \int_{-\infty}^{\infty}\left|J_{g r}(x)\right|^{2} d x
$$

where $J_{g r}$ is the current density on the ground plane given in reference [133], and $R_{s}$ is the Leontovich surface impedance [139] is given by eq (50). This integral can be evaluated to yield

$$
\alpha_{g r}=\frac{R_{s}}{w \pi Z_{0}}\left\{\arctan \frac{w}{2 h}-\frac{h}{w} \ln \left(1+\left(\frac{w}{2 h}\right)^{2}\right)\right\} .
$$

Thus, the total conductor loss is given by

$$
\alpha_{T(m i c r o)}=\alpha_{s t r i p}+\alpha_{g r}
$$

\subsubsection{Stripline}

In stripline, as shown in figure 32 , the field is confined primarily to the region between ground planes. Striplines have been used both in resonant and nonresonant modes 
of operation. A commonly used technique is the stripline-card method [119]. In this technique, a stripline is etched into a large sheet of PWB material. The stripline is then resonated and, thereby, an estimate of the permittivity is obtained. In another approach, the specimen sheet and copper conductors are stacked and then pressure is applied to form a stripline resonator [140].

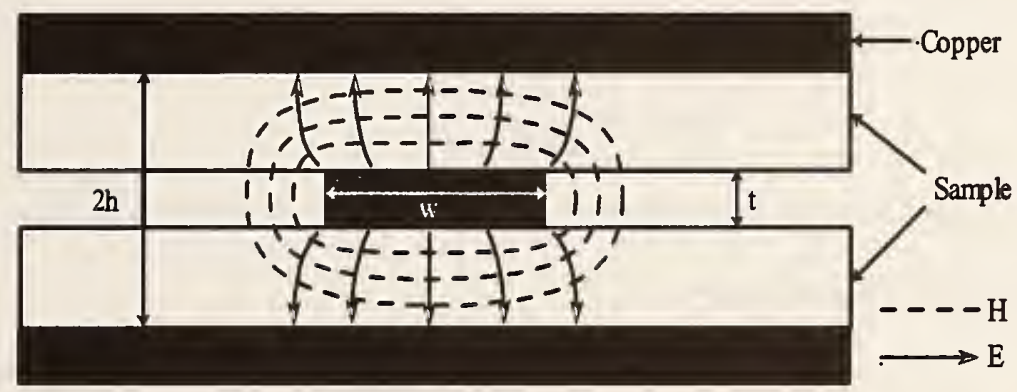

Figure 32. Cross section of a stripline resonator [141].

In stripline, the TEM mode resonates at frequencies corresponding to integral multiples of one-half wavelength. $\epsilon_{r}^{\prime}$ for stripline is given by

$$
\epsilon_{r}^{\prime}=\left[\frac{n c}{2 f_{r}(L+\Delta L)}\right]^{2},
$$

where $n$ is the number of half wavelengths, $c$ is the speed of light, $L$ is the physical length of transmission line, $\Delta L$ is an effective length extension due to fringing fields, and $f_{r}$ is the resonance frequency. The loss tangent is calculated from

$$
\tan \delta=\frac{1}{Q_{\text {unl }}}+\frac{1}{Q_{c}},
$$

where $Q$ unl is the quality factor not including coupling port losses, but includes losses due to the dielectric, and the metal, and $Q_{c}$ is the quality factor of the coupling probes $[127,142]$. 
As with the microstrip, the line loss for a stripline consists of components from both the strip and its ground planes. Holloway has calculated the attenuation constant for stripline $[133,136]$, which is

$$
\alpha_{T(\text { stripline })}=\alpha_{\text {strip }}+\alpha_{\text {ground }}
$$

where

$$
\alpha_{s t r i p}=\frac{R_{s m}}{2 \pi^{2} Z_{0} w} \ln \left(\frac{w}{\Delta}-1\right) .
$$

Once again, $\Delta$ is the stopping distance and is given in table 19. $R_{s m}$ is given by eq (72) and $Z_{0}$ is the impedance

$$
Z_{0}=\sqrt{\frac{\mu_{0}}{16 \epsilon}} \frac{K(k)}{K\left(k^{\prime}\right)},
$$

where $K$ is the elliptical integral of the first kind, $k=\operatorname{sech}(\pi w / 4 h), k^{\prime}=\sqrt{1-k^{2}}, w$ is the width of strip, and $h$ is the distance between the inner and outer conductors. The ground losses are

$$
\alpha_{\text {ground }}=\frac{R_{s}}{Z_{0} w} F\left(\frac{w}{h}\right),
$$

where $R_{s}=1 / \sigma \delta$ is the surface impedance, $\sigma$ is the metal conductivity, and $\delta$ is the skin depth in conductor, and

$$
F\left(\frac{w}{h}\right)=\frac{2 h}{\pi^{2} w} \int_{-\infty}^{\infty}\left[\tan ^{-1}\left(e^{(y-w \pi / 4 h)}\right)-\tan ^{-1}\left(e^{(y+w \pi / 4 h)}\right)\right]^{2} d y .
$$

A plot of $F(w / h)$ for different values of $w / h$ is given in reference [133]. Variation of attenuation with frequency, metal thickness, and conductivity for a stripline are shown in figures 33 through 37.

The dielectric losses vary as

$$
\alpha_{d}=\frac{\omega \sqrt{\epsilon_{0} \mu_{0}}}{2} \sqrt{\epsilon_{r}} \tan \delta
$$




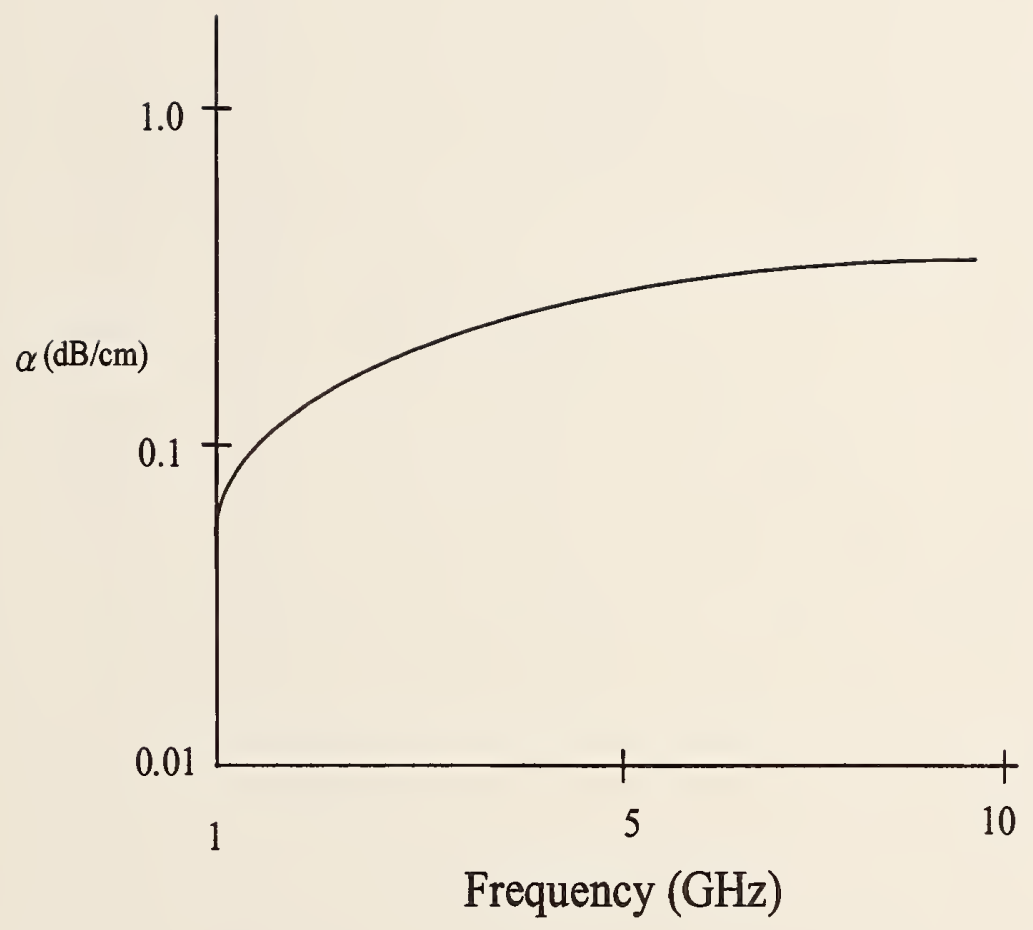

Figure 33. Total attenuation of dielectric, ground plane, and strip as a function of frequency for a stripline line with geometries having $t=10 \mu \mathrm{m}, w=200 \mu \mathrm{m}$, $h=455 \mu \mathrm{m}, \tan \delta=0.005, \sigma=2.5 \times 10^{7} \mathrm{~S} / \mathrm{m}[136]$. 


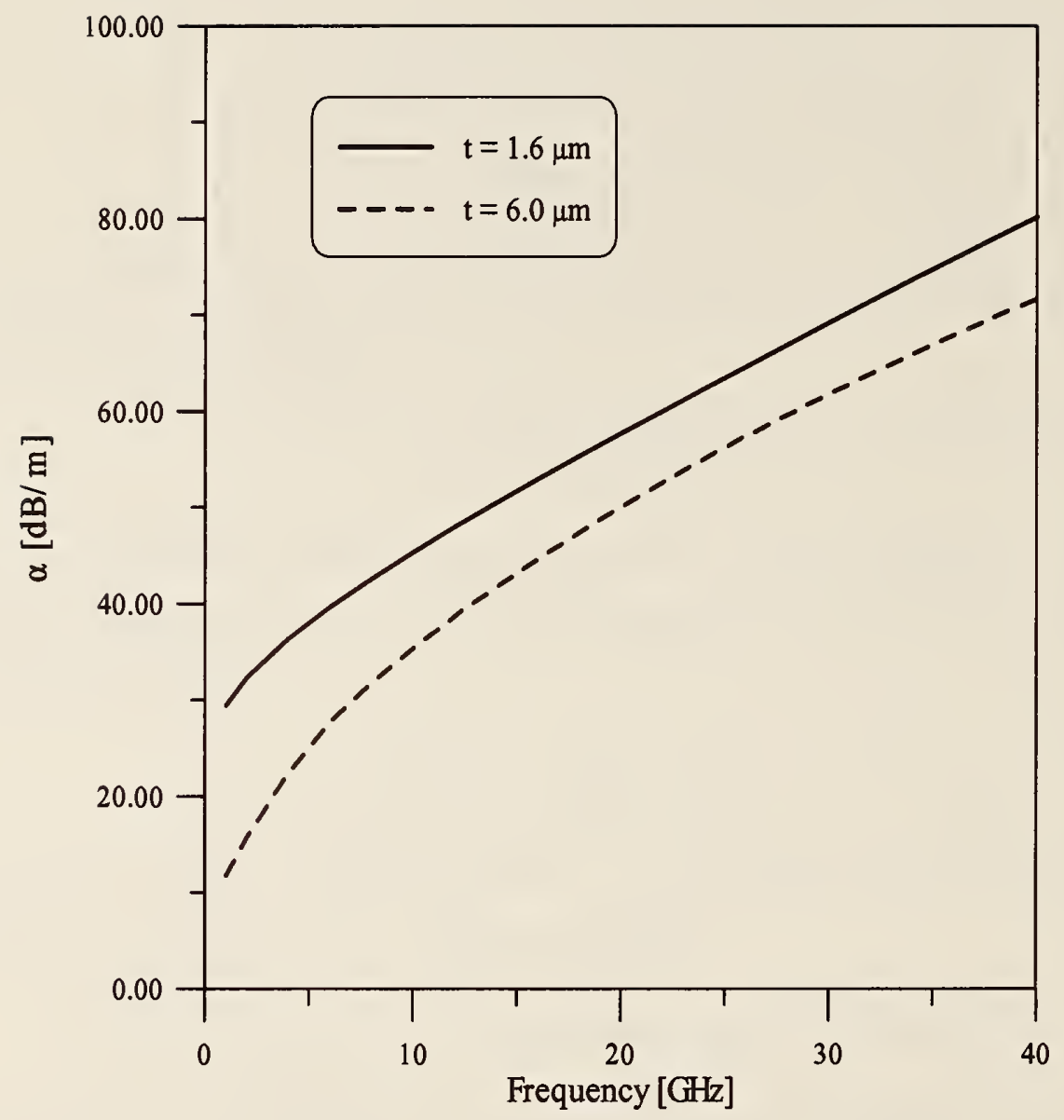

Figure 34. Attenuation for two different thicknesses $(t)$ plotted against frequency for a stripline with geometries having $h=100 \mu \mathrm{m}, w=70 \mu \mathrm{m}, \epsilon_{r}^{\prime}=12.9, \sigma=$ $3.6 \times 10^{7} \mathrm{~S} / \mathrm{m}$. 


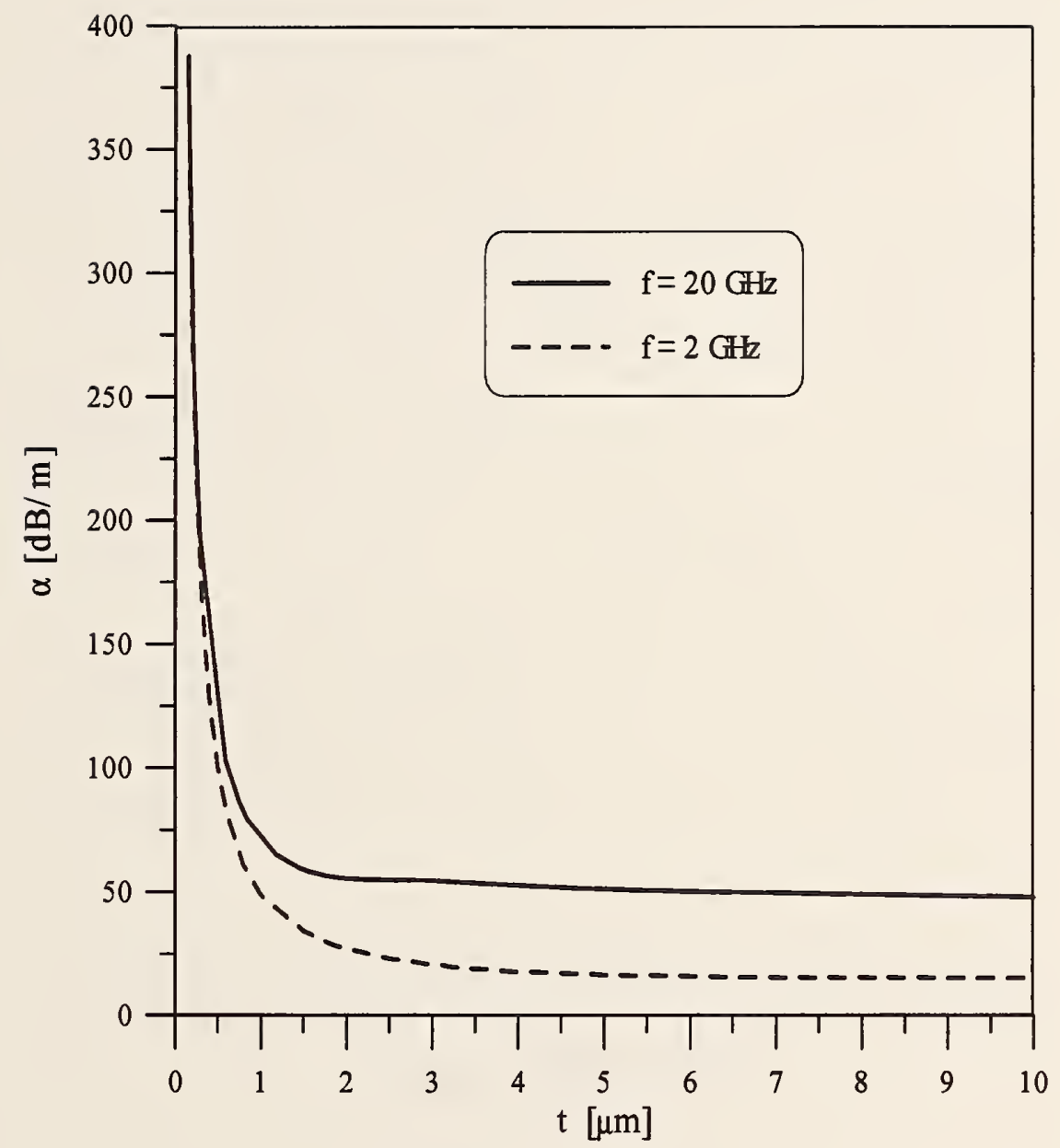

Figure 35. Attenuation for two frequencies plotted against thickness for a stripline with geometries having $h=100 \mu \mathrm{m}, w=70 \mu \mathrm{m}, \epsilon_{r}^{\prime}=12.9, \sigma=3.6 \times 10^{7} \mathrm{~S} / \mathrm{m}$. 


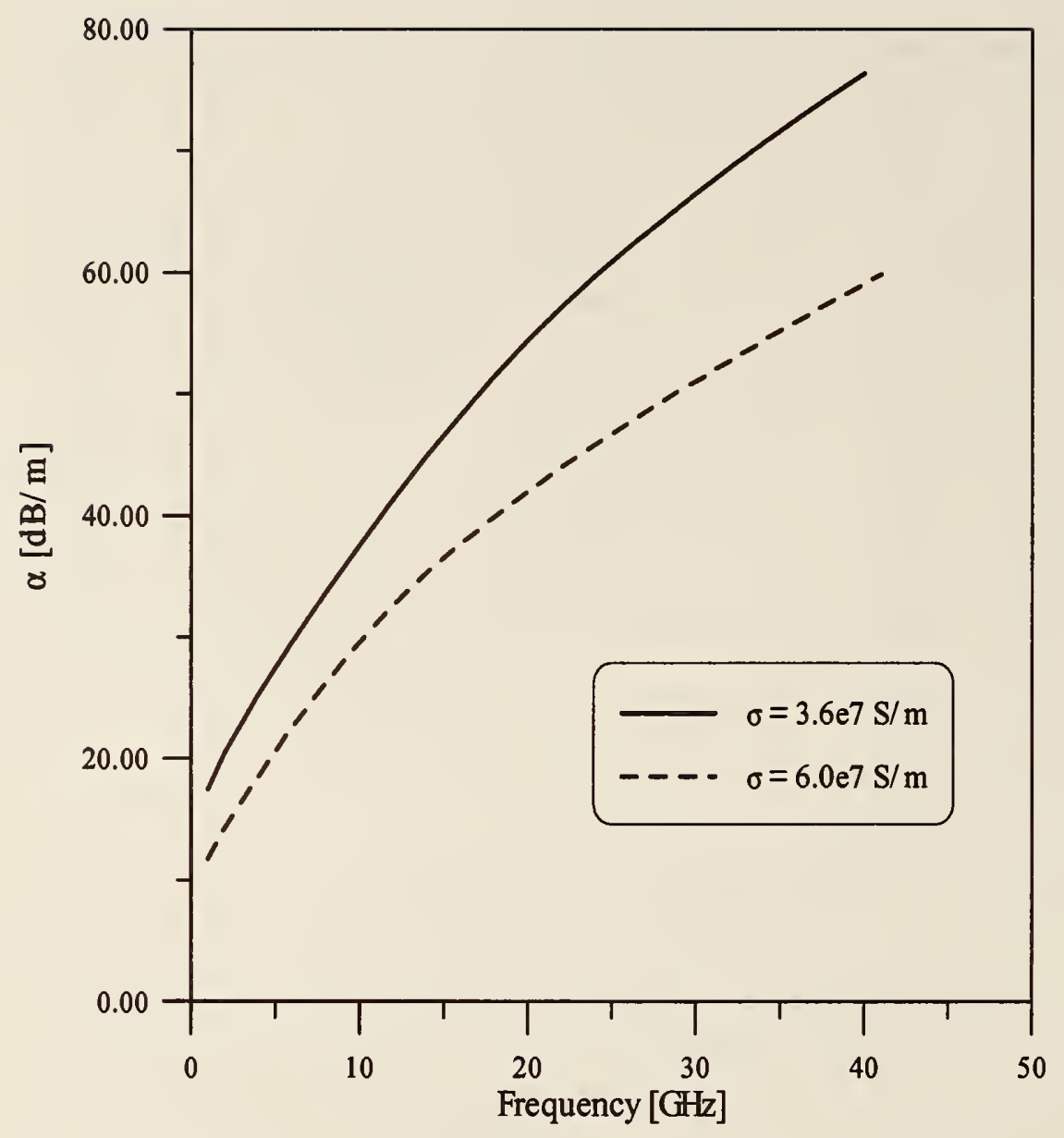

Figure 36. Dependence of attenuation for two frequencies as a function of metal conductivities for a stripline with geometries having $h=100 \mu \mathrm{m}, w=70 \mu \mathrm{m}, \epsilon_{r}^{\prime}=$ $12.9, t=3 \mu \mathrm{m}$. 


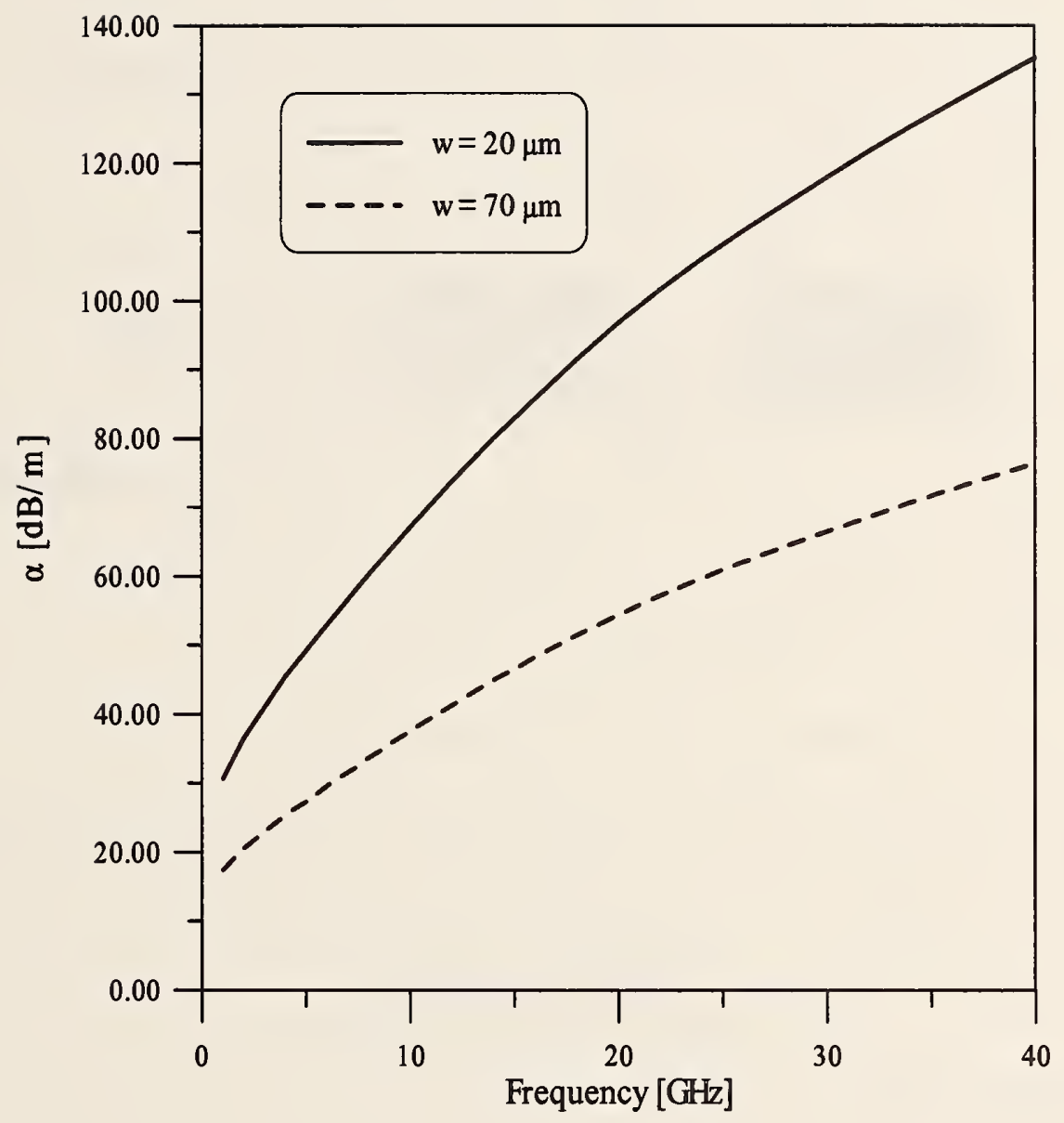

Figure 37. Dependence of attenuation at two frequencies on frequency for various conductor widths for a stripline with geometries having $h=100 \mu \mathrm{m}, t=3 \mu \mathrm{m}$, $\epsilon_{r}^{\prime}=12.9, t=3 \mu \mathrm{m}, \sigma=3.6 \times 10^{7} \mathrm{~S} / \mathrm{m}$. 


\subsubsection{Coplanar Strip (CPS)}

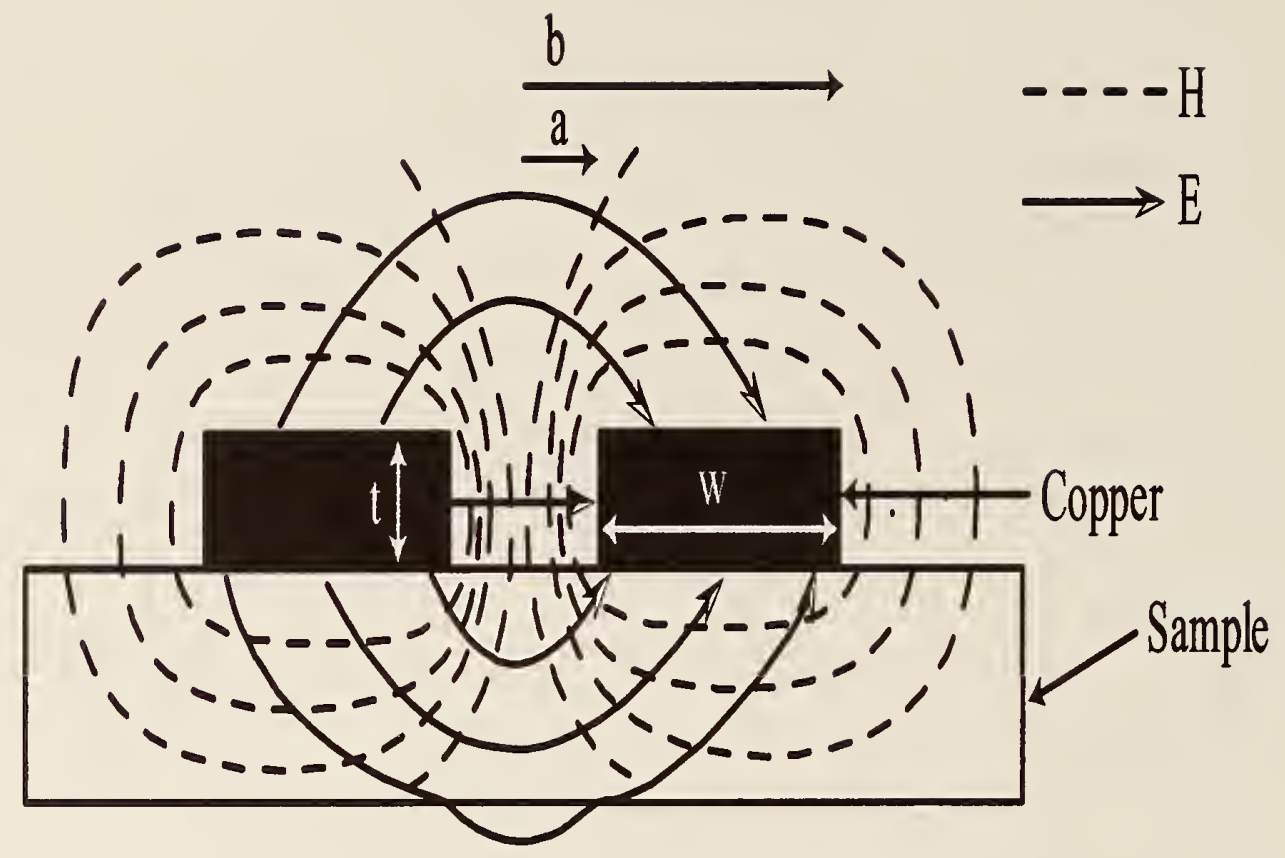

Figure 38. Coplanar strip cross section.

In CPS, as shown in figure 38 , the characteristic impedance is given by [136]

$$
Z_{0}=\sqrt{\frac{2 \mu_{0}}{\epsilon_{0}\left(\epsilon_{r}+1\right)}} \frac{K(k)}{K\left(\sqrt{1-k^{2}}\right)} .
$$

The attenuation is [136]:

$$
\alpha_{C P S} \approx \frac{R_{s m} b^{2}}{4 Z_{0} K^{2}\left(k^{\prime}\right)\left(b^{2}-a^{2}\right)}\left[\frac{1}{a} \ln \left(\frac{2 a+\Delta}{\Delta} \frac{b-a-\Delta}{b+a-\Delta}\right)+\frac{1}{b} \ln \left(\frac{2 b-\Delta}{\Delta} \frac{b-a-\Delta}{b+a+\Delta}\right)\right] .
$$

Variation of attenuation with frequency for CPS is shown in figure 39. 


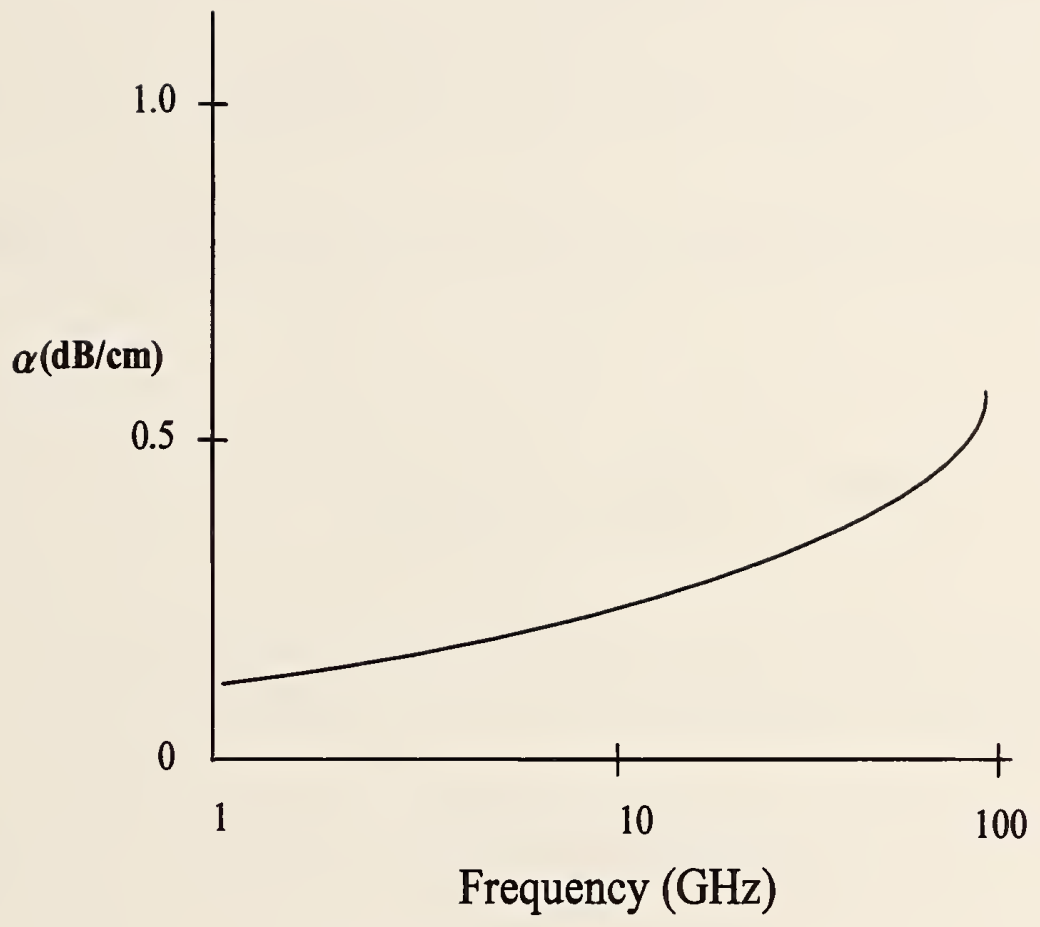

Figure 39. Total attenuation plotted against frequency for a CPS line with $t=1.5 \mu \mathrm{m}$, $a=10 \mu \mathrm{m}, b=25 \mu \mathrm{m}, \epsilon=12.9, \sigma=3 \times 10^{7} \mathrm{~S} / \mathrm{m}[136]$. 


\subsubsection{Coplanar Waveguide (CPW)}

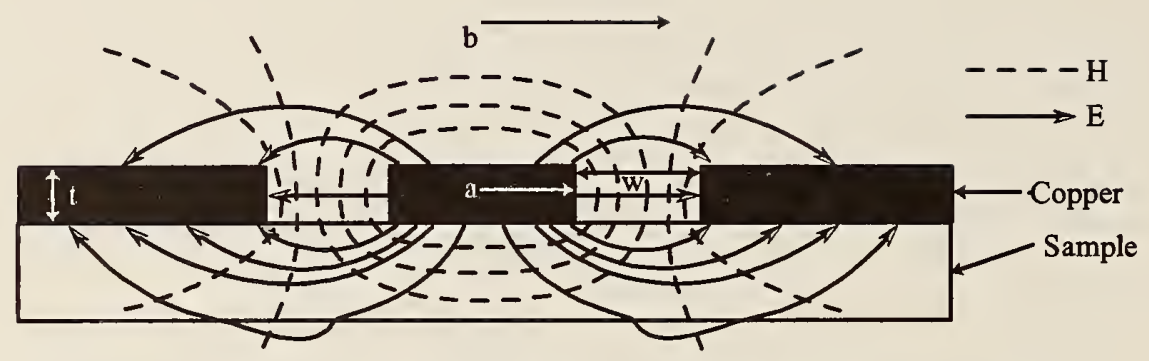

Figure 40. Coplanar waveguide fixture cross section.

The attenuation in CPW (see figures 40 through 43) was calculated by Holloway $[134,135]$

$$
\alpha_{C P W} \approx \operatorname{Re}\left[\frac{F}{Z_{0}} \frac{2}{1+\sqrt{1+\frac{4 F}{Z_{0} \gamma_{m o}}}}\right]
$$

where

$$
F=\frac{\left(Z_{s}+Z_{m}\right) b^{2}}{16 K^{2}(k)\left(b^{2}-a^{2}\right)}\left[\frac{1}{a} \ln \left(\frac{2 a}{\Delta} \frac{b-a}{b+a}\right)+\frac{1}{b} \ln \left(\frac{2 b}{\Delta} \frac{b-a}{b+a}\right)\right],
$$

and

$$
\begin{gathered}
\gamma_{m o}=j \omega \sqrt{\epsilon \mu_{0}} \sqrt{\frac{\epsilon_{r}+1}{2}}, \\
Z_{0}=\sqrt{\frac{\mu_{0}}{16 \epsilon_{0} \epsilon_{e f f}} \frac{K\left(k^{\prime}\right)}{K(k)},} \\
\epsilon_{e f f}=\frac{\epsilon_{r}+1}{2} \\
Z_{s}=-j \sqrt{\frac{\mu_{0}}{\epsilon_{c}-j \sigma / \omega}} \cot \left(k_{c} t\right), \\
Z_{m}=-j \sqrt{\frac{\mu_{0}}{\epsilon_{c}-j \sigma / \omega}} \csc \left(k_{c} t\right),
\end{gathered}
$$

and $\sigma$ is the conductor's conductivity, $\epsilon_{c}$ is the conductor's permittivity, and

$$
k^{\prime}=\sqrt{1-k^{2}} .
$$




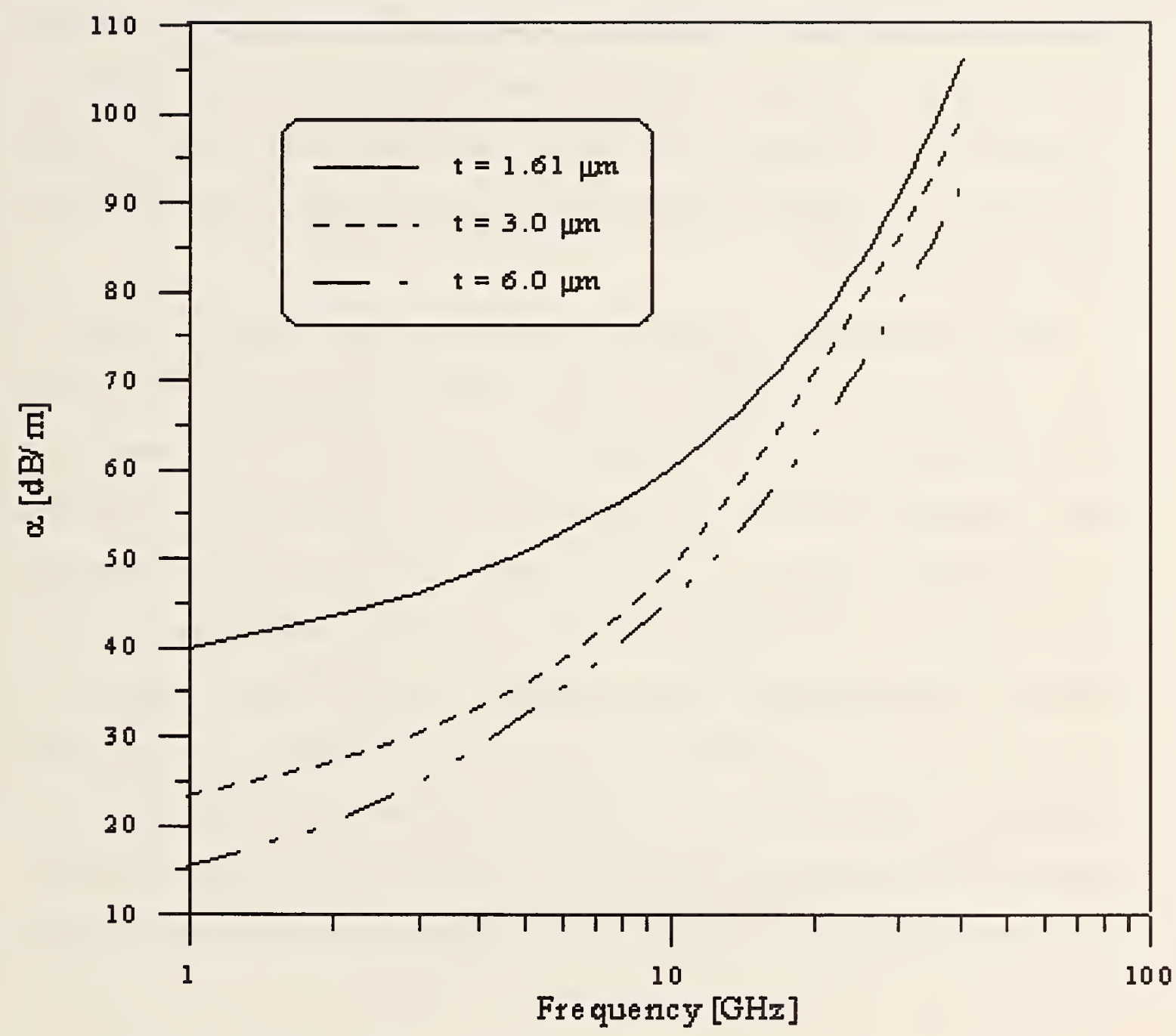

Figure 41. Attenuation for three thicknesses $(\mathrm{t})$ plotted against frequency for a CPW line with geometries having $a=35.6 \mu \mathrm{m}, b=84.6 \mu \mathrm{m}, \epsilon_{r}^{\prime}=12.9, \sigma=3.6 \times 10^{7} \mathrm{~S} / \mathrm{m}$.

\subsubsection{Increased Power Loss Associated with Surface Roughness}

If the surfaces of the conductors are rough, then the conductor's power loss increases.

The amount of increase will depend on the direction of the roughness with respect to 
the current flow. A recent study discussing these effects is given in reference [143]. Figures 45 through 48 show the increase in power loss $(P)$ of a rough conductor relative to that $\left(P_{0}\right)$ of a smooth conductor for two different polarizations and different roughness profiles as a function of $p / \delta$, where $\delta$ is skin depth and $p$ is effective length characterizing the roughness. These results are for the two-dimensional rectangular profile shown in figure 44 .

It was shown that as the roughness dimensions get large compared to the skin depth, there are noticeable roughness effects. Typical surface roughness cannot be represented accurately by two-dimensional periodic profiles, but are instead more likely to have three-dimensional, random profiles. For very small ratios of the period to skin-depth $(p / \delta)$, there is no physical reason to expect that the more general random profiles will exhibit drastically different $P / P_{o}$ behavior from that of the twodimensional periodic surfaces. From our analysis of two-dimensional roughness, we see that for large $p / \delta$, the additional power loss resulting from a rough interface can be significant. While quantitative predictions of this loss cannot be made from our analysis of the two-dimensional profiles for randomly rough surfaces, we can conclude that at these relatively large values of $p / \delta$, the additional power loss associated with a randomly rough surface is also expected to be significant. 


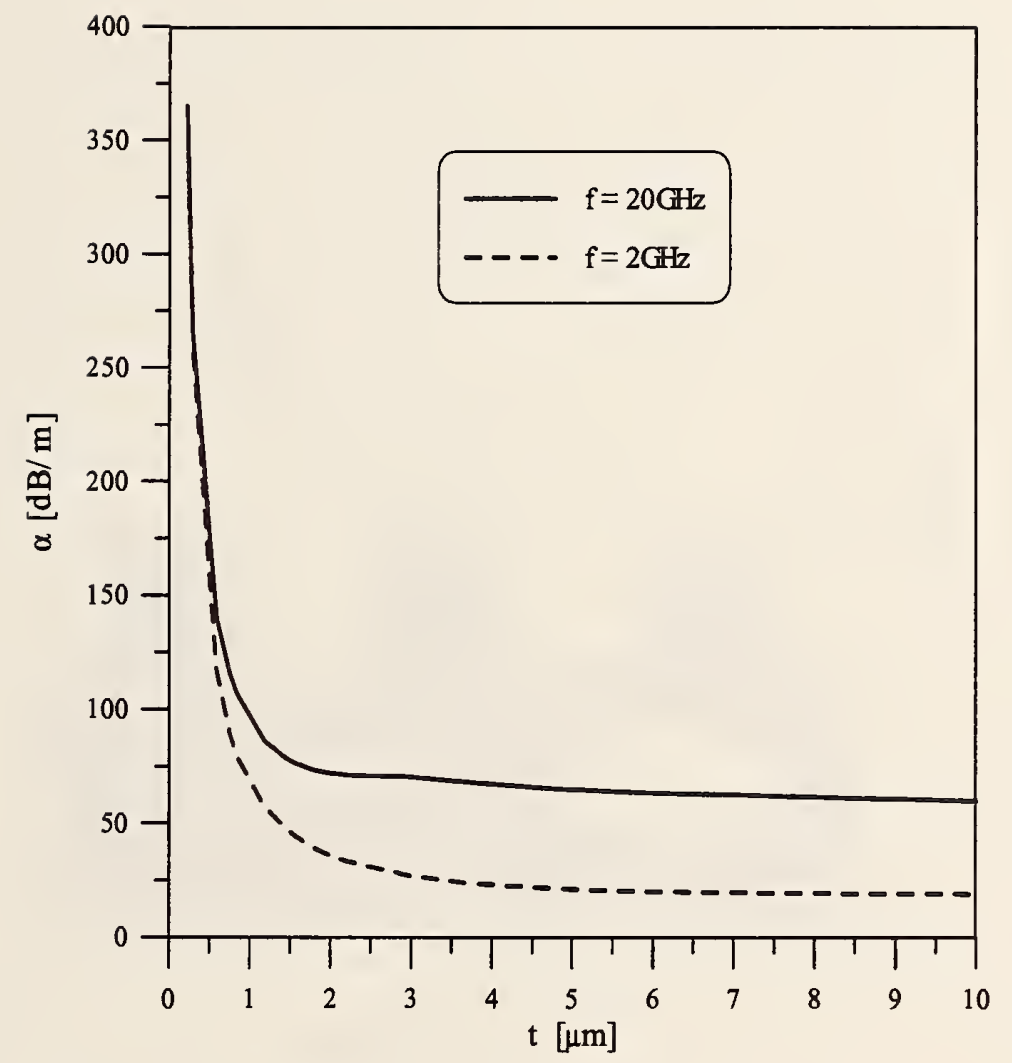

Figure 42. Attenuation as a function of thickness of conductor $(\mathrm{t})$ for two frequencies for a CPW line with geometries having $a=35.6 \mu \mathrm{m}, b=84.6 \mu \mathrm{m}, \epsilon_{r}^{\prime}=12.9, \sigma=$ $3.6 \times 10^{7} \mathrm{~S} / \mathrm{m}$ as a function of thickness $(\mathrm{t})$. 


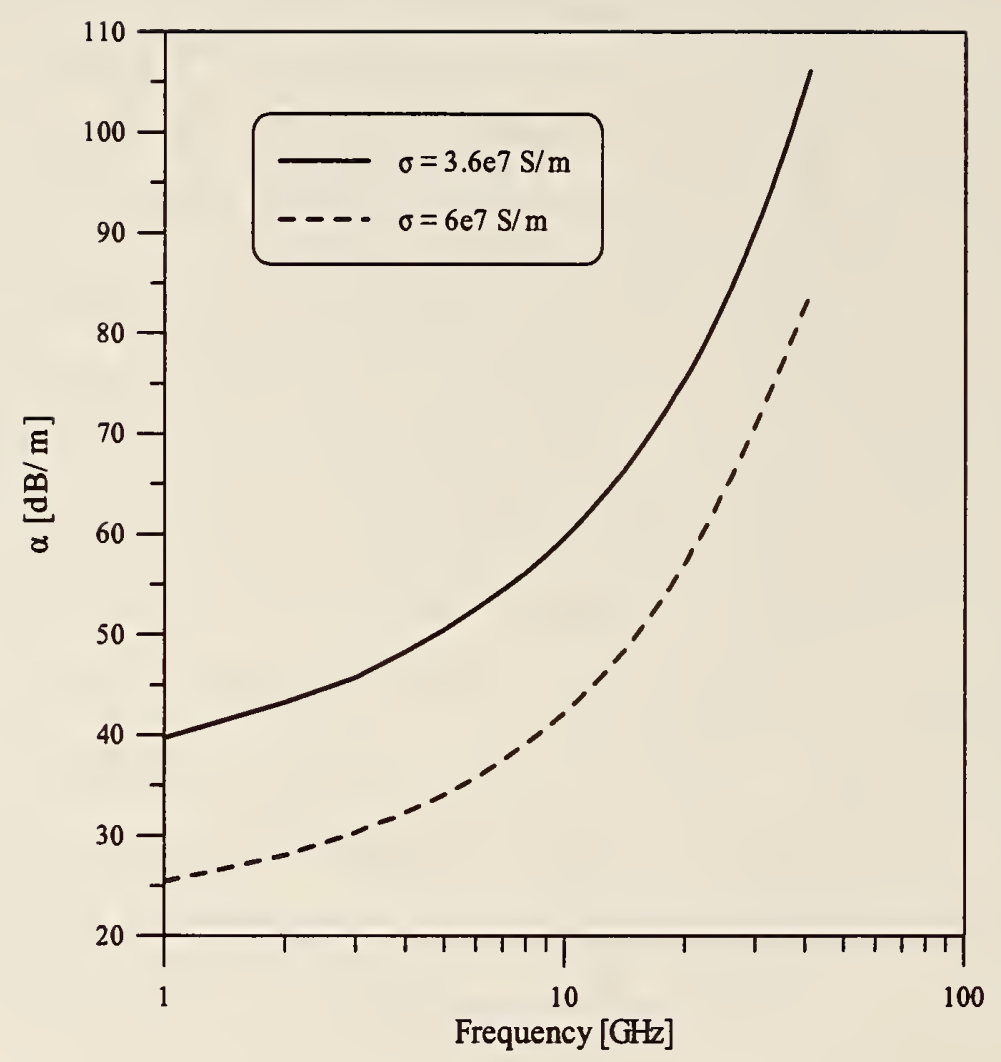

Figure 43. Attenuation as a function of frequency for two different conductivities for a CPW line with geometries having $t=3 \mu \mathrm{m}, a=35.6 \mu \mathrm{m}, b=84.6 \mu \mathrm{m}, \epsilon_{r}^{\prime}=12.9$. 


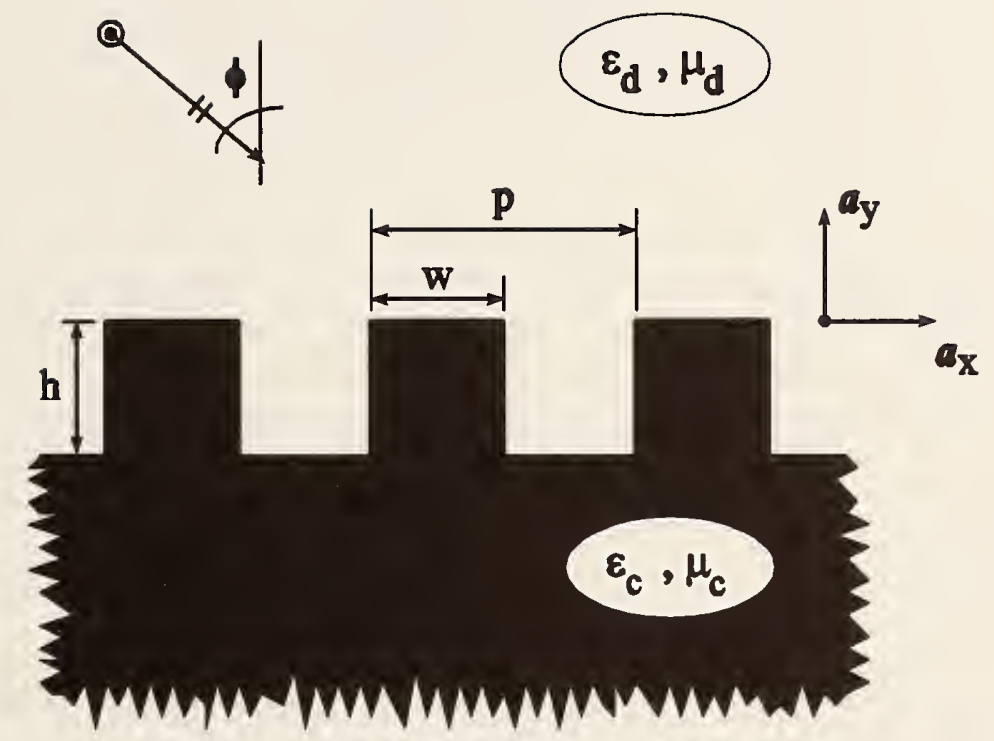

Figure 44. Two-dimensional rectangular profile. 


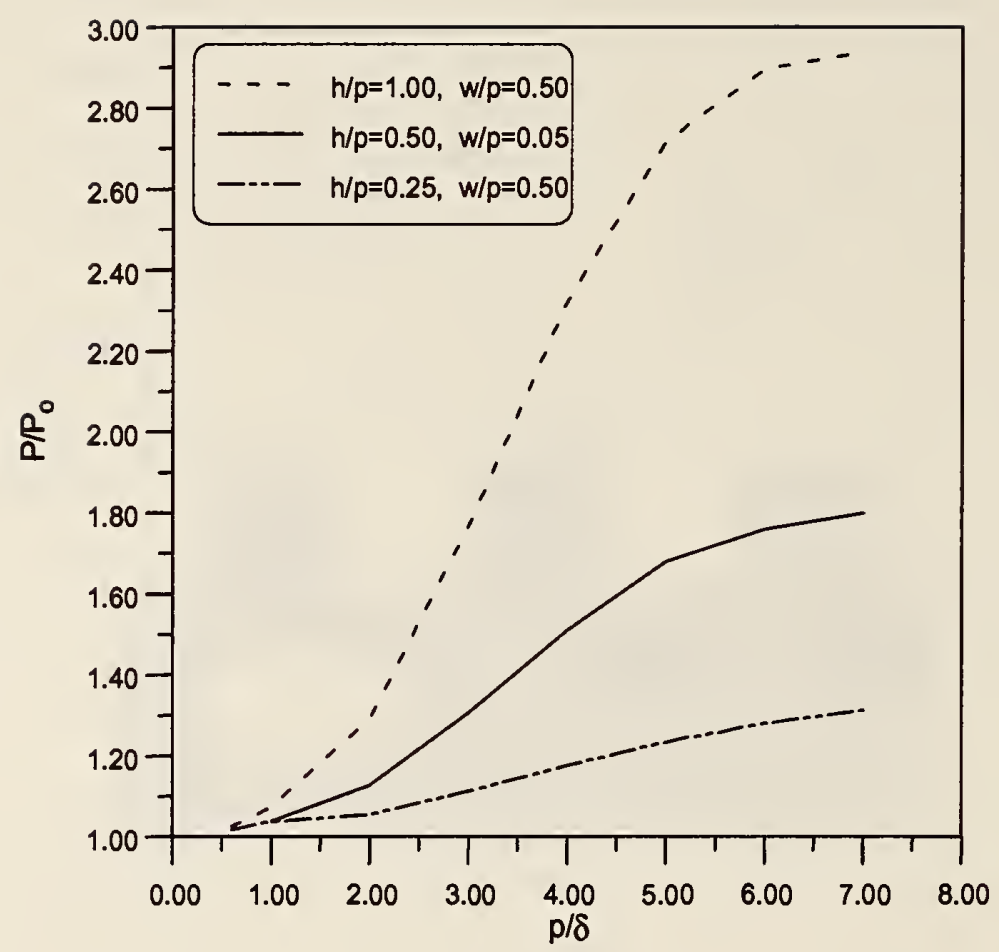

Figure 45. Power ratio for grooves transverse to current flow with $\frac{w}{p}=0.5$ and $\frac{h}{p}$ equal to $0.25,0.5$, and 1.0 . 


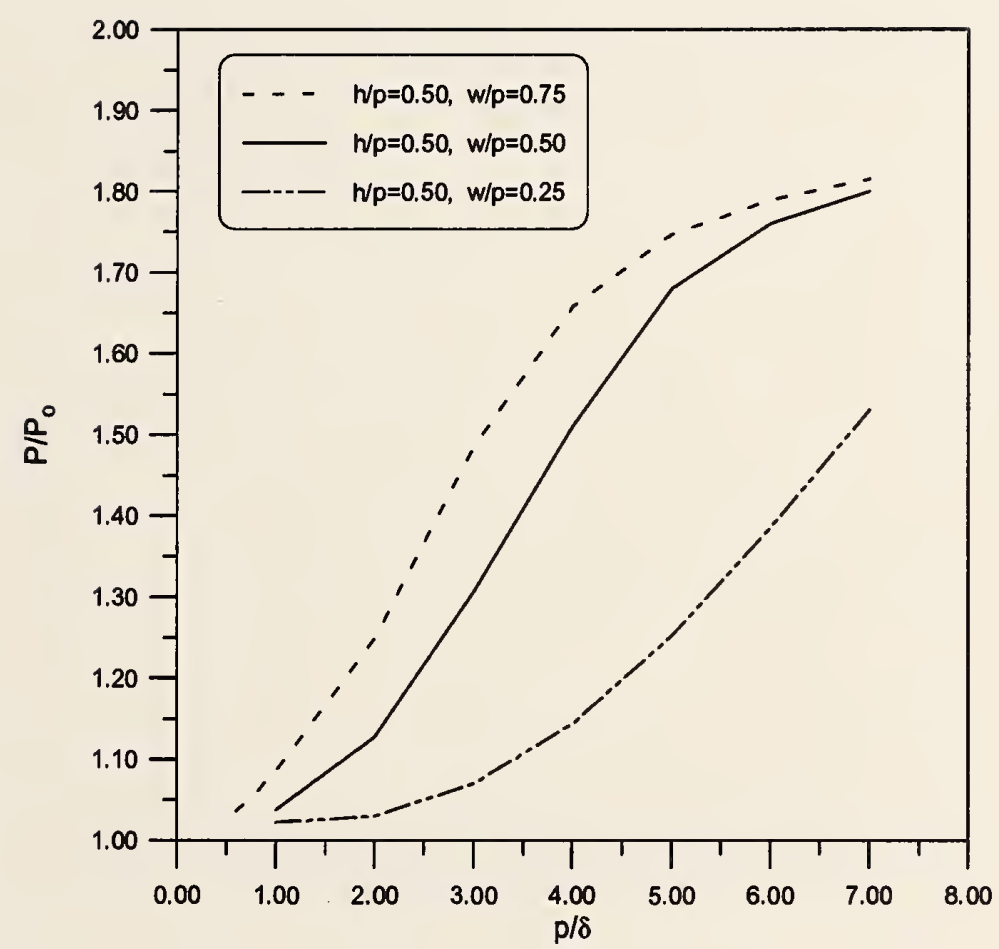

Figure 46. Power ratio for grooves transverse to current flow with $\frac{h}{p}=0.5$ and $\frac{w}{p}$ equal to $0.25,0.5$, and 0.75 . 


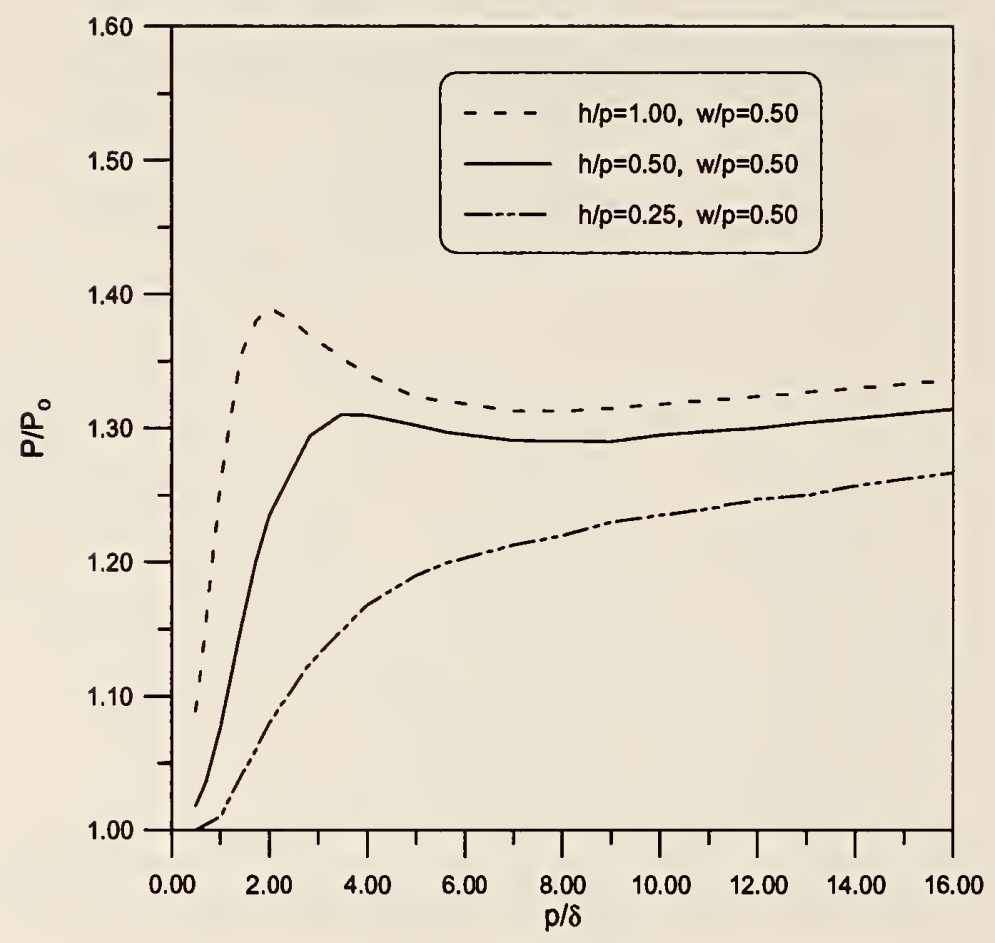

Figure 47. Power ratio for grooves parallel to current flow with $\frac{w}{p}=0.5$ and $\frac{h}{p}$ equal to $0.25,0.5$, and 1.0 . 


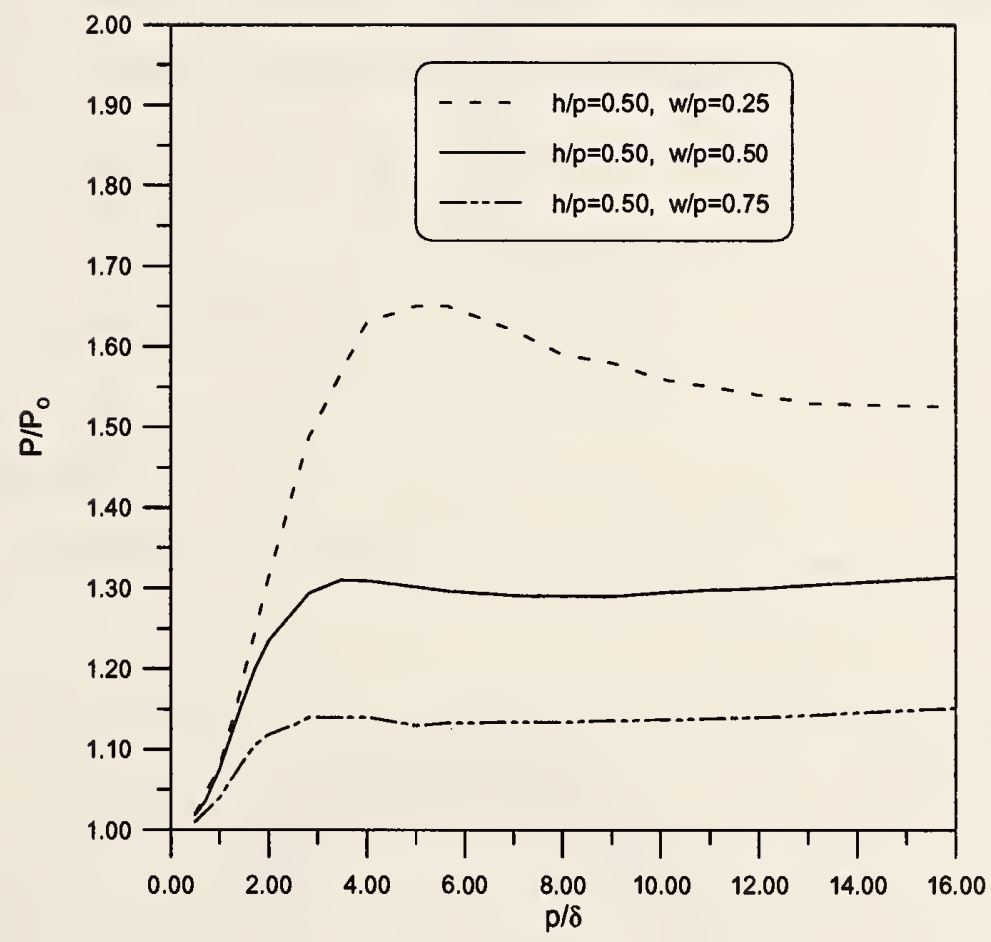

Figure 48. Power ratio for grooves parallel to current flow with $\frac{h}{p}=0.5$ and $\frac{w}{p}$ equal to $0.25,0.5$, and 0.75 . 


\subsection{T and Ring-Resonator Methods for Substrates}

The $\mathrm{T}$ and ring-resonator methods have been developed in order to obtain relatively broadband measurements using small, resonating devices (see figures 49 and 50). Broadband permittivity measurements using the ring resonator can be obtained by use of higher-order modes. However, accuracy of these measurement techniques degrades for the high modes [144]. For the ring resonator, usually a very simplistic model of medium accuracy is used which neglects radiation [145].

$$
\epsilon_{r}^{\prime}=\left(\frac{n c}{\pi<d>f}\right)^{2}
$$

where $n$ is the number of half wavelengths, $f$ is frequency, $\langle d\rangle$ is the mean diameter, and $c$ is the speed of light.

A T-resonator length correction is commonly performed (see Amey et al. [144]). The open-ended transmission-line stub in the T-resonator resonates at integral multiples of one-quarter wavelength.

Measurement of substrate loss is limited to $\tan \delta \geq 0.001$ with these methods. Simple models usually suffice for characterizing the ring resonator; however, the system can be modeled by a Green function method that yields an integral equation for the permittivity. This equation must be solved numerically for the permittivity [145-147].

\subsection{On-Wafer Transmission-line Methods for Substrates}

Using on-wafer transmission-line techniques, one can measure the broadband permittivity and loss tangent of substrates in the microwave region. Specifically, from measurements of the propagation constant and characteristic impedance of a coplanar waveguide on a dielectric substrate, one can determine the permittivity of the 


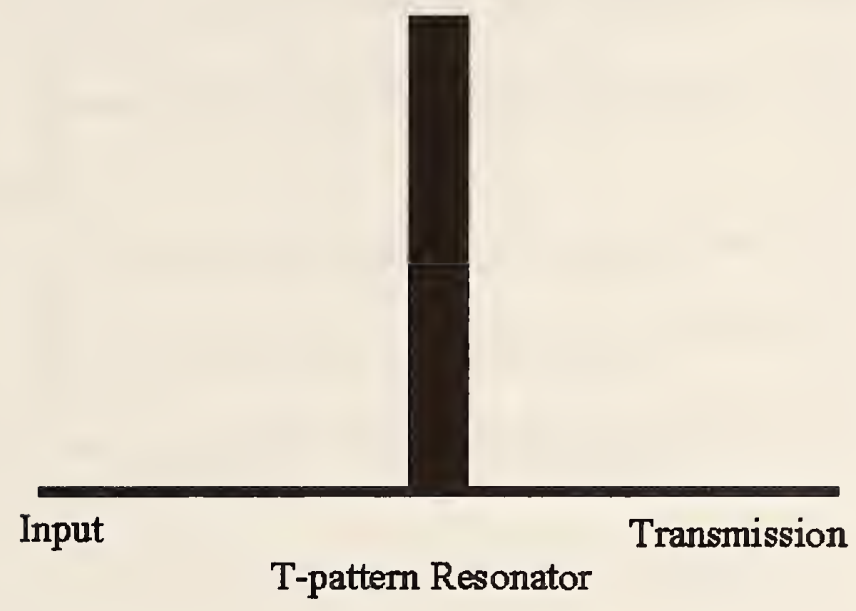

Figure 49. T-resonator.

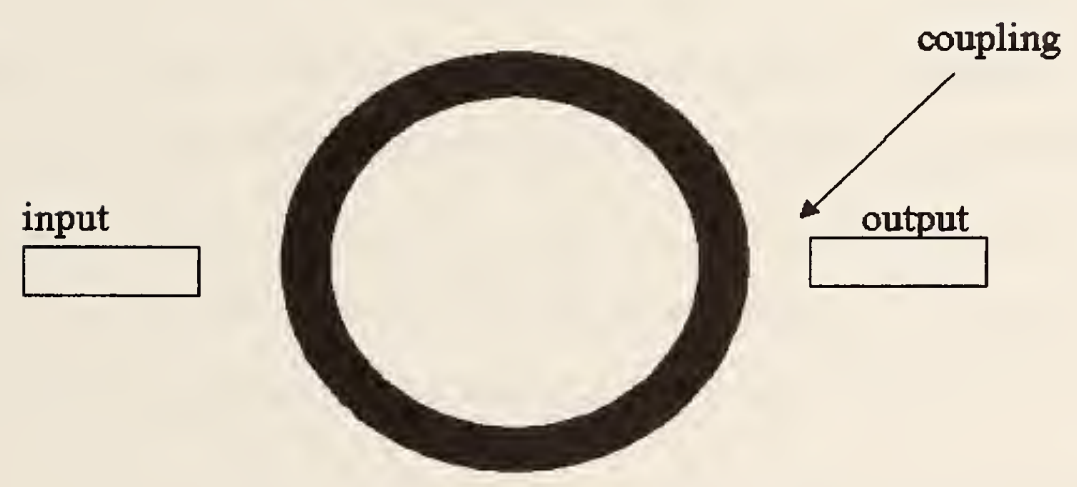

Ring Resonator

Figure 50. Ring resonator. 


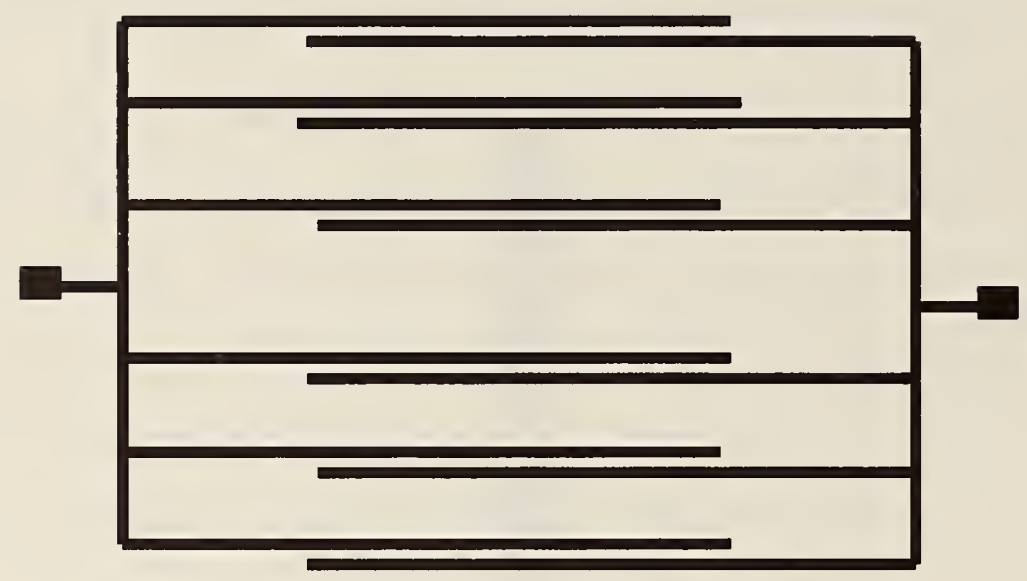

Figure 51. Interdigitated capacitor.

substrate. Reference [32] provides measurements results for several low-loss dielectric substrates over the frequency range of $50 \mathrm{MHz}$ to $40 \mathrm{GHz}$. Although these methods are broadband, the measurement uncertainty is higher than those for single-frequency resonator methods, especially for the loss tangent. This is due mainly to the fact that the conductive losses in the transmission line are usually larger than the dielectric losses in the substrate. Although one can separate the two losses, the measurement sensitivity is decreased, leading to a larger uncertainty in the substrate's loss tangent.

At lower frequencies, substrate permittivity has been measured using planar interdigitated capacitors, as shown in figure 51. This structure uses the many fingers to increase the capacitance and hence decrease the measurement uncertainty [148].

\section{Dielectric Measurement Software}

Software has been developed in the Electromagnetic Properties of Materials Project at NIST, Boulder. Table 20 summarizes the available software at the time of publication. 
Table 20. Software summary.

\begin{tabular}{c} 
Transmission line (coaxial line and waveguide) \\
Coaxial probe \\
Shielded open-circuited holder \\
Reentrant cavity \\
Split-cylinder \\
Permeameter \\
Courtney \\
\hline
\end{tabular}

\section{Ambient Temperature Measurements}

Figures 52 and 53 show results using the split-cylinder and split-post resonators on FR-4. The darker square at $9 \mathrm{GHz}$ is a measurement from another spatial position on the same sample.

In figures 54 and 55, the results of measurements on a sample of aluminum nitride are shown using various measurement fixtures. Note that the loss tangent decreases as frequency increases above $3 \mathrm{GHz}$.

Figures 56 and 57 plot the permittivity of high-resistivity gallium arsenide as a function of frequency. These measurements were made by a mode-filtered $\mathrm{TE}_{01}$ X-band cavity. 


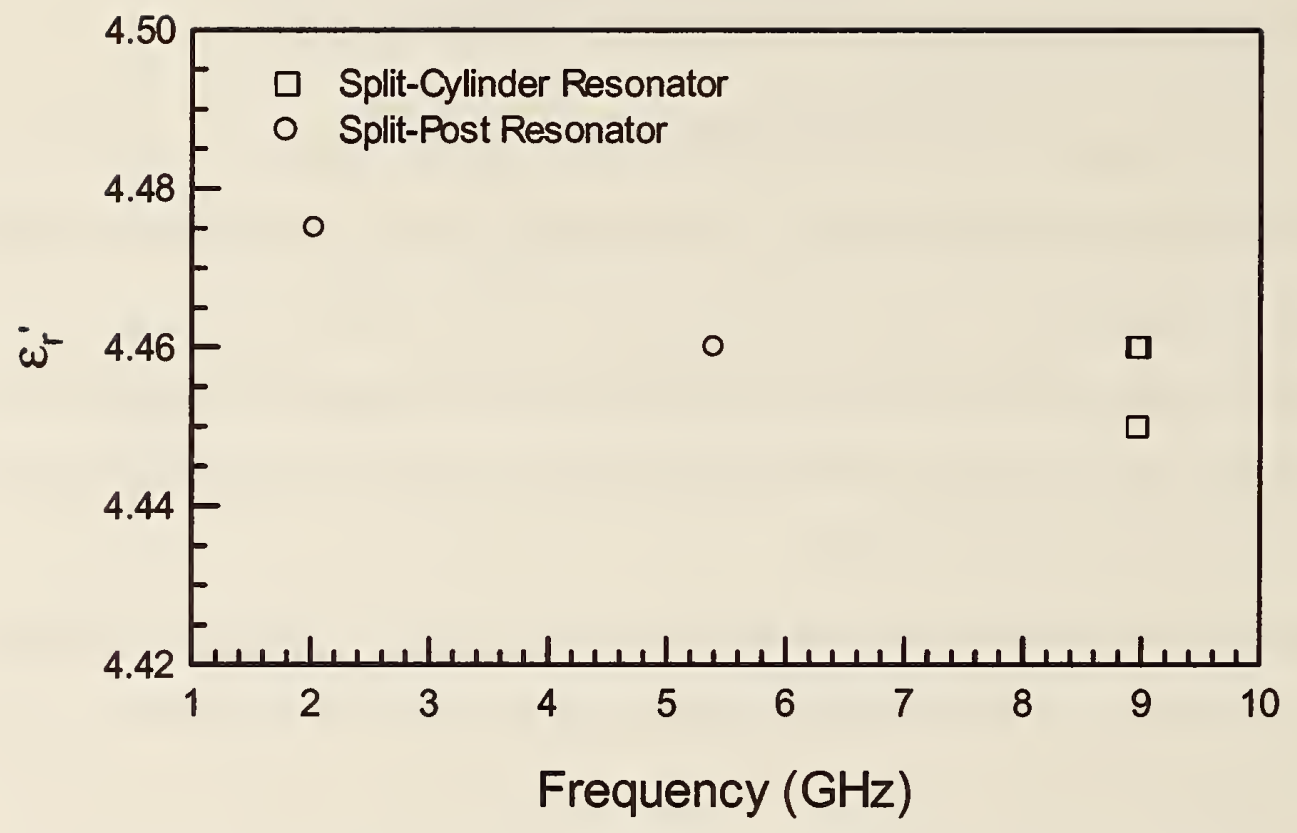

Figure 52. Measurements of the real part of the permittivity of FR-4. 


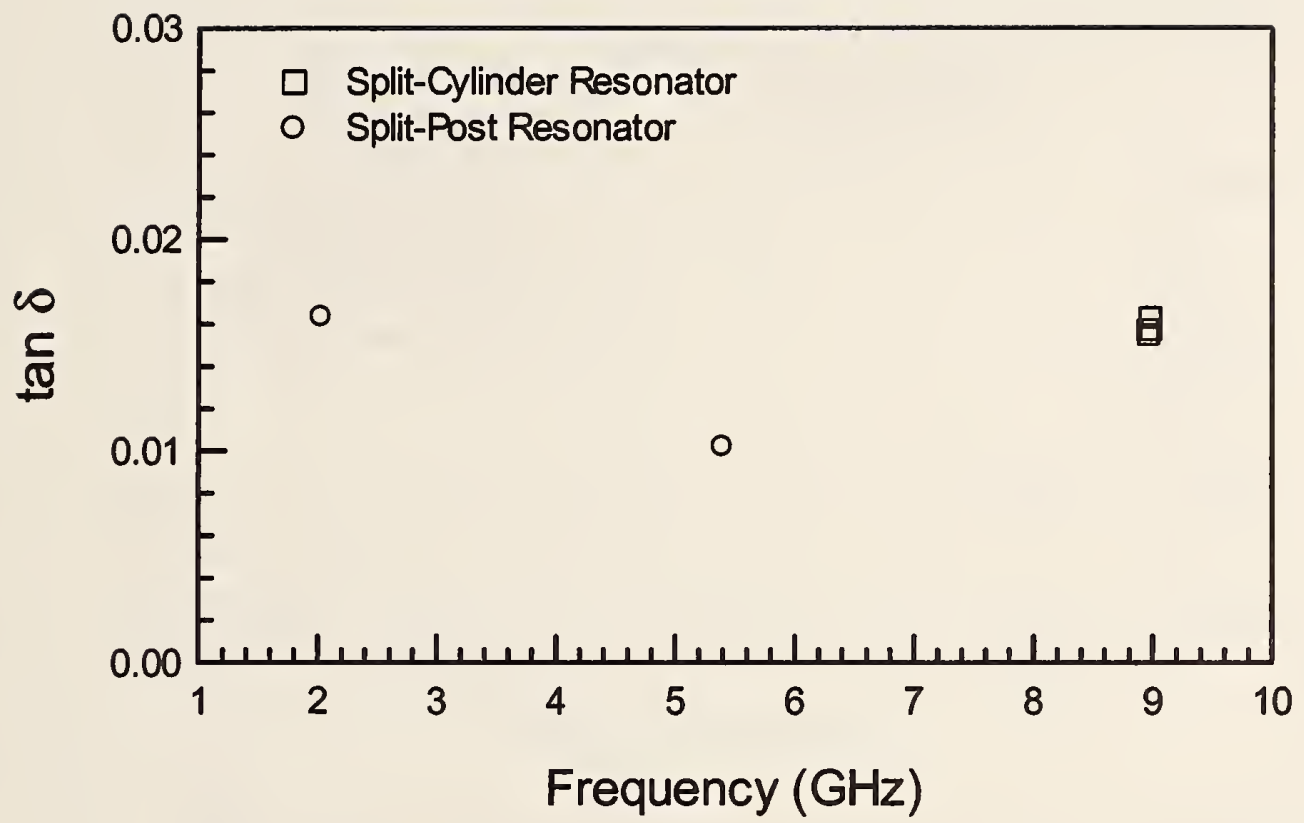

Figure 53. Loss tangent of FR-4.

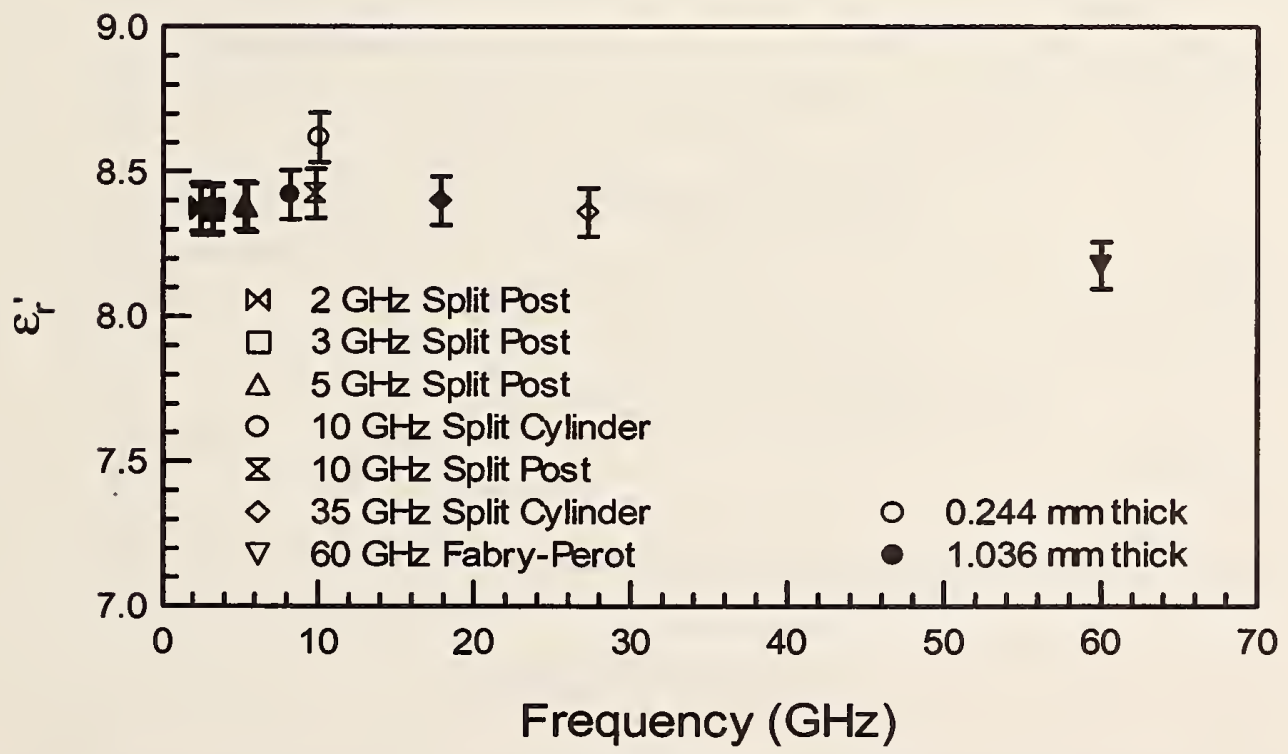

Figure 54. Measurements of the real part of aluminum nitride from 2 to $60 \mathrm{GHz}$. 


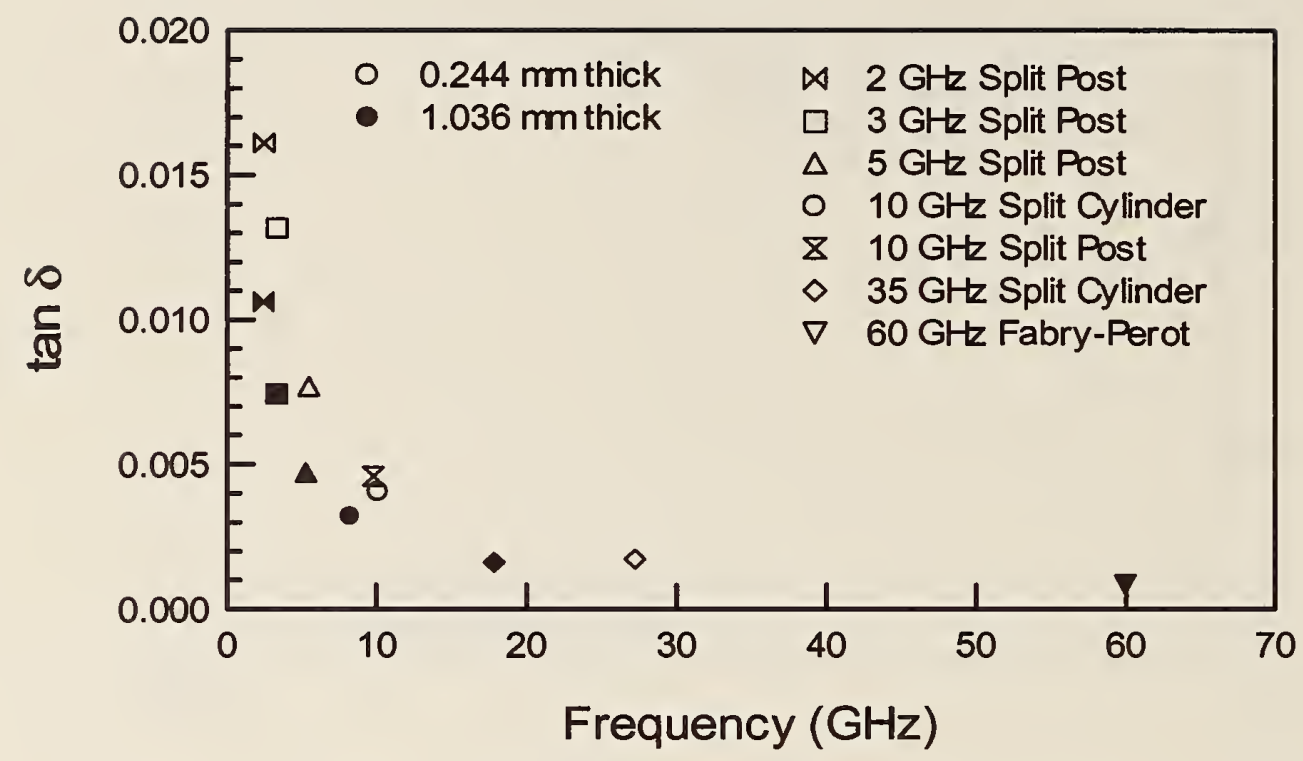

Figure 55. Loss tangent of aluminum nitride from 2 to $60 \mathrm{GHz}$.

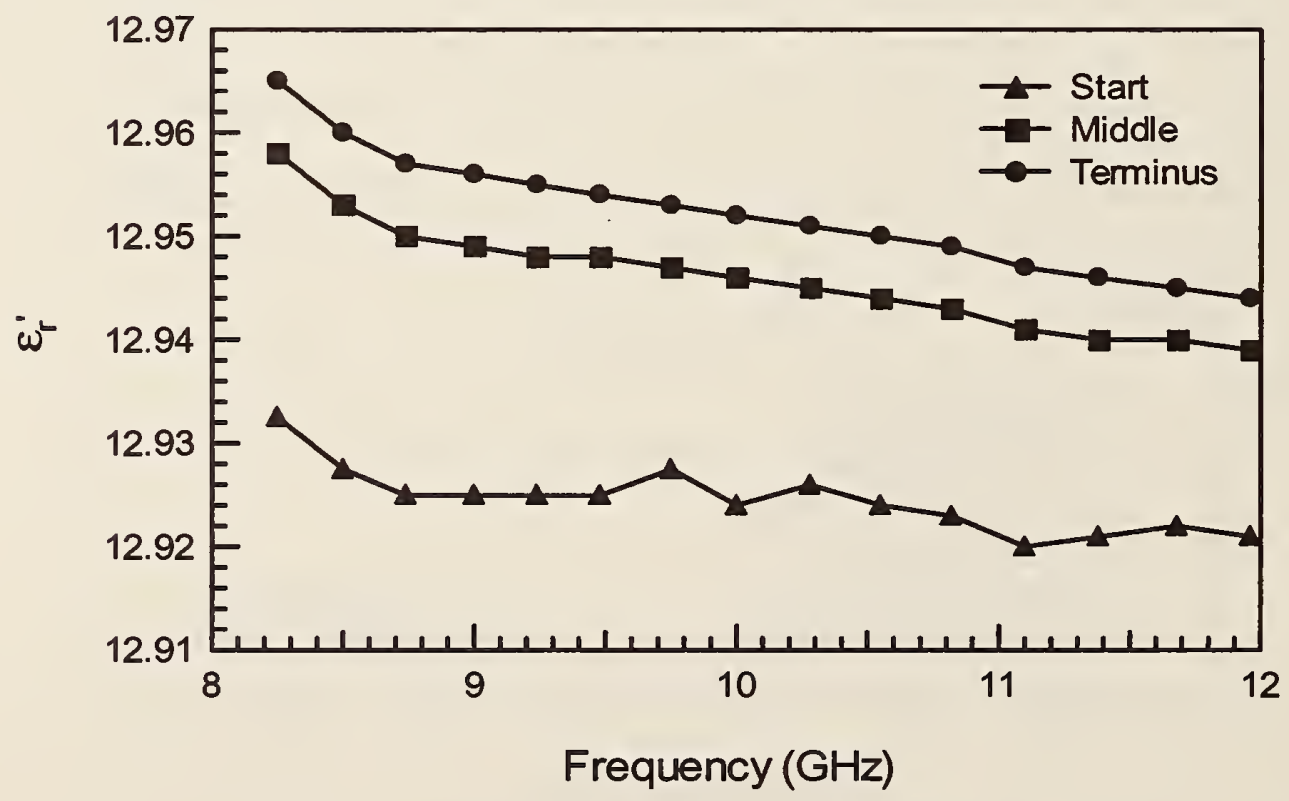

Figure 56. $\epsilon_{r}^{\prime}$ of gallium arsenide using an X-band cavity [149]. Start, middle, and terminus refer to different specimens taken from the same boule. 


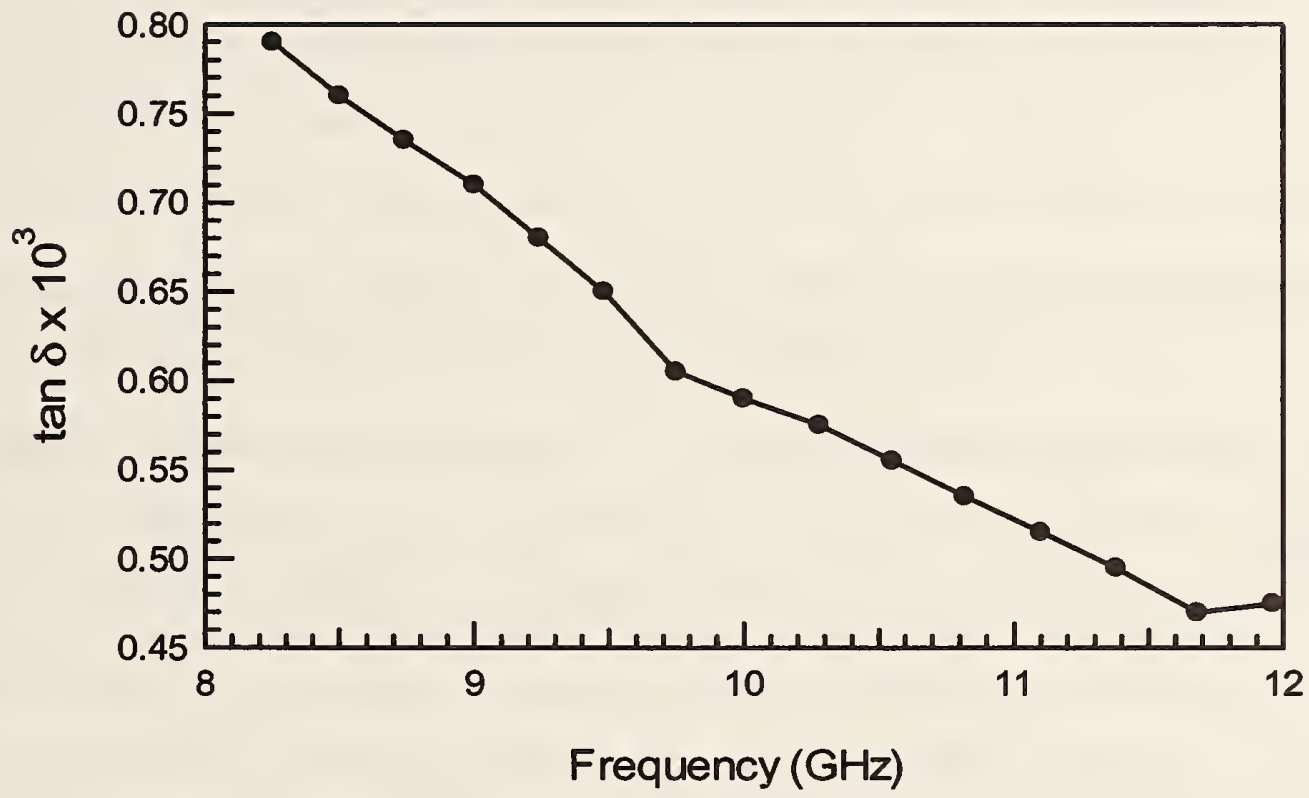

Figure 57. Loss tangent of gallium arsenide using an X-band cavity [149]. 
Table 21. Split-post dielectric resonator measurement for low-loss materials [150]. The alumina are from different manufacturers.

\begin{tabular}{c|ccccccc}
\hline Material & $\mathrm{f}(\mathrm{MHz})$ & $\mathrm{Q}$ & $\mathrm{h}(\mathrm{mm})$ & $\epsilon_{r}^{\prime}$ & $\tan \delta\left(\times 10^{4}\right)$ & $U\left(\epsilon_{r}^{\prime}\right), k=2$ & $U(\tan \delta)\left(\times 10^{4}\right)$ \\
\hline Teflon & 5587.07 & 9040 & 0.620 & 2.04 & 2.7 & \pm 0.03 & \pm 1.0 \\
$\mathrm{Al}_{2} \mathrm{O}_{3}$ & 5559.31 & 9639 & 0.842 & 9.95 & 0.4 & \pm 0.03 & \pm 0.5 \\
$\mathrm{Al}_{2} \mathrm{O}_{3}$ & 5321.06 & 9115 & 0.999 & 9.75 & 1.0 & \pm 0.06 & \pm 0.5 \\
$\mathrm{Al}_{2} \mathrm{O}_{3}$ & 5406.28 & 8081 & 0.740 & 9.23 & 2.7 & \pm 0.06 & \pm 0.5 \\
\hline
\end{tabular}

Measurement results for different materials using a split-post dielectric resonator operating at a frequency of approximately $5.6 \mathrm{GHz}$ with associated uncertainties are shown in tables 21 through 25 [151]. In table 21, three alumina samples from three manufacturers are given. Notice the variation in results. This is due to density variations between the various alumina samples. Measurement results for stacked or layered dielectrics using this method are nearly independent of the number of layers (see table 21). This is a result of the field being in the plane of the sample for the split dielectric resonator method and, therefore, it is insensitive to the presence of air gaps between stacked films. Some dielectric substrates may exhibit uniaxial anisotropy. Therefore, additional measurements in the direction perpendicular to the substrate are necessary to characterize fully the dielectric properties. Measurement of the component of permittivity normal to the sample plane can also be accomplished using the reentrant cavity technique.

In table 25 measurements on PWB materials using the split-post resonator method are displayed. Tables 26 to 32 report measurements of many materials often used for substrates. The results are presented as additional data on substrate materials reproduced from reference [151]. 
Table 22. Measurements with split-post dielectric resonators operating unloaded at 9.6 and $12.6 \mathrm{GHz}$ [150].

\begin{tabular}{c|ccccccc}
\hline Material & $\mathrm{f}(\mathrm{MHz})$ & $\mathrm{Q}$ & $\mathrm{h}(\mathrm{mm})$ & $\epsilon_{r}^{\prime}$ & $\tan \delta\left(\times 10^{4}\right)$ & $\mathrm{U}\left(\epsilon_{r}^{\prime}\right), \mathrm{k}=2$ & $\mathrm{U}\left(\tan \delta \times 10^{4}\right)$ \\
\hline $\mathrm{Al}_{2} \mathrm{O}_{3}$ (Vistal) & 9570.2 & 9740 & 0.85 & 10.0 & 0.24 & \pm 0.05 & \pm 0.5 \\
Rexolite & 10214 & 7666 & 0.98 & 2.53 & 4.16 & \pm 0.02 & \pm 1 \\
$\mathrm{GaAs}$ & 9719.5 & 7134 & 0.51 & 12.9 & 2.75 & \pm 0.02 & \pm 1 \\
$\mathrm{Teflon}$ & 10304 & 8709 & 0.62 & 2.05 & 1.61 & \pm 0.03 & \pm 1 \\
$\mathrm{Teflon}$ & 12727 & 5416 & 0.62 & 2.03 & 1.94 & \pm 0.05 & \pm 1 \\
$\mathrm{LaSrAlO}_{3}$ & 11319 & 5081 & 0.50 & 23.2 & 1.78 & \pm 0.07 & \pm 1 \\
$\mathrm{Al}_{2} \mathrm{O}_{3}$ & 11955 & 5560 & 0.70 & 9.94 & 1.18 & \pm 0.05 & \pm 0.5 \\
\hline
\end{tabular}

Table 23. Measurement on polymer films from split-post dielectric resonators operating unloaded at nominally $1.46 \mathrm{GHz}$ and $2.05 \mathrm{GHz}$ [150].

\begin{tabular}{c|ccccccc}
\hline Material & $\mathrm{f}(\mathrm{MHz})$ & $\mathrm{Q}$ & $\mathrm{h}(\mathrm{mm})$ & $\epsilon_{r}^{\prime}$ & $\tan \delta\left(\times 10^{4}\right)$ & $U\left(\epsilon_{r}^{\prime}\right)$ & $U\left(\tan \delta \times 10^{4}\right)$ \\
film & 2046.00 & 2020.7 & 0.96 & 3.34 & 324 & \pm 0.02 & \pm 50 \\
film & 1455.27 & 2487.6 & 0.96 & 3.34 & 323 & \pm 0.02 & \pm 50 \\
\hline
\end{tabular}

Table 24. Split-post (in-plane) measurements on Rogers PWB materials

\begin{tabular}{c|ccccc}
\hline Material & Frequency $(\mathrm{GHz})$ & $\epsilon_{r}^{\prime}$ & $\mathrm{U}\left(\epsilon_{r}^{\prime}, \mathrm{k}=2\right)$ & $\tan \delta$ & $\mathrm{U}(\tan \delta)$ \\
\hline RO3003 & 3.36 & 2.96 & \pm 0.015 & $9.6 \times 10^{-4}$ & $5 \times 10^{-5}$ \\
RO3003 & 3.36 & 3.01 & \pm 0.015 & $8.7 \times 10^{-4}$ & $5 \times 10^{-5}$ \\
RO4350(Lot 1) & 3.36 & 3.61 & \pm 0.015 & $3.7 \times 10^{-3}$ & $5 \times 10^{-5}$ \\
RO4350(Lot 2) & 3.36 & 3.75 & \pm 0.015 & $3.8 \times 10^{-3}$ & $5 \times 10^{-5}$ \\
RO4003 & 3.36 & 3.55 & \pm 0.015 & $2.3 \times 10^{-3}$ & $5 \times 10^{-5}$ \\
RO3003 & 3.36 & 3.60 & \pm 0.015 & $2.4 \times 10^{-3}$ & $5 \times 10^{-5}$ \\
RO3010(Lot 1) & 3.36 & 11.2 & \pm 0.025 & $1.4 \times 10^{-3}$ & $5 \times 10^{-5}$ \\
RO3010(Lot 2) & 3.36 & 11.4 & \pm 0.025 & $2.5 \times 10^{-3}$ & $5 \times 10^{-5}$ \\
RO3010(Lot 3) & 3.36 & 11.5 & \pm 0.025 & $2.5 \times 10^{-3}$ & $5 \times 10^{-5}$ \\
\hline
\end{tabular}


Table 25. Split dielectric resonator measurements on PWB materials [150].

\begin{tabular}{c|cccccccc}
\hline Specimen & $\mathrm{f}(\mathrm{MHz})$ & $\mathrm{Q}$ & $\mathrm{h}(\mathrm{mm})$ & $\epsilon_{r}^{\prime}$ & $\tan \delta\left(\times 10^{4}\right)$ & $U_{\epsilon}$, & $U_{t}, k=2\left(\times 10^{4}\right)$ \\
\hline & 5608.71 & 8515 & & & & \pm 0.02 & \pm 4 \\
1 & 5584.71 & 2823 & 0.402 & 2.77 & 177 & \pm 0.02 & \pm 4 \\
2 & 5585.05 & 2829 & 0.412 & 2.71 & 176 & \pm 0.02 & \pm 4 \\
3 & 5584.66 & 2828 & 0.402 & 2.78 & 176 & \pm 0.02 & \pm 4 \\
4 & 5584.66 & 2818 & 0.399 & 2.79 & 177 & \pm 0.02 & \pm 4 \\
5 & 5585.21 & 2825 & 0.405 & 2.73 & 178 & \pm 0.02 & \pm 4 \\
6 & 5582.34 & 5561 & 0.393 & 3.28 & 46.5 & \pm 0.02 & \pm 1 \\
7 & 5579.97 & 5413 & 0.380 & 3.25 & 45.9 & \pm 0.02 & \pm 1 \\
8 & 5579.41 & 5346 & 0.380 & 3.29 & 46.8 & \pm 0.02 & \pm 1 \\
\hline
\end{tabular}

Table 26. Measurements on glass specimens. The expanded uncertainties, $k=2$ are as follows: parallel-plate dielectric resonator: $U_{\epsilon^{\prime}}=0.5 \%, U_{\tan \delta}=2 \times 10^{-5}$, split-cylinder: $U_{\epsilon^{\prime}}=1 \%, U_{\tan \delta}=1 \times 10^{-4}, \mathrm{TE}_{01}$ cavity resonator: $U_{\epsilon^{\prime}}=1 \%$, $U_{\tan \delta}=1 \times 10^{-4}$, Fabry-Perot resonator: $U_{\epsilon^{\prime}}= \pm 2 \%, U_{\tan \delta}=2 \times 10^{-4}$, reentrant cavity: $U_{\epsilon^{\prime}}= \pm 1 \%, U_{\tan \delta}=2 \times 10^{-4}$.

\begin{tabular}{c|cccccc}
\hline Material & Value & Parallel plate & Split cavity & $60 \mathrm{~mm} \mathrm{TE} \mathrm{m}_{01}$ & Split post & Reentrant \\
\hline Corning & $\mathrm{f}(\mathrm{GHz})$ & 5.07 & 8.85 & 9.64 & 2.03 & \\
7980 & $\epsilon^{\prime}$ & 3.84 & 3.827 & 3.84 & 3.84 & \\
34604 & $\tan \delta$ & $6.85 \times 10^{-5}$ & $11.9 \times 10^{-5}$ & $1.40 \times 10^{-4}$ & $4.40 \times 10^{-5}$ & \\
\hline Corning & $\mathrm{f}(\mathrm{GHz})$ & 5.07 & 8.82 & 9.63 & 2.03 & \\
7980 & $\epsilon^{\prime}$ & 3.844 & 3.822 & 3.843 & 3.838 & \\
34605 & $\tan _{\delta}$ & $6.8 \times 10^{-5}$ & $11.7 \times 10^{-5}$ & $1.4 \times 10^{-4}$ & $3.1 \times 10^{-5}$ & \\
\hline Corning & $\mathrm{f}(\mathrm{GHz})$ & 5.26 & 8.86 & 9.64 & 2.03 & \\
7980 & $\epsilon^{\prime}$ & 3.844 & 3.825 & 3.826 & 3.84 & \\
34606 & $\tan \delta$ & $6.90 \times 10^{-5}$ & $1.80 \times 10^{-4}$ & $1.5 \times 10^{-4}$ & $4.9 \times 10^{-5}$ & \\
\hline Corning & $\mathrm{f}(\mathrm{GHz})$ & 4.89 & 8.86 & 9.64 & 2.03 & \\
7940 & $\epsilon^{\prime}$ & 3.847 & 3.825 & 3.826 & 3.839 & \\
44608 & $\tan \delta$ & $6.83 \times 10^{-5}$ & $11.6 \times 10^{-5}$ & $1.4 \times 10^{-4}$ & $3.6 \times 10^{-5}$ & \\
\hline Corning & $\mathrm{f}(\mathrm{GHz})$ & 9.0 & & & & 1.0 \\
H. Bussey & $\epsilon^{\prime}$ & 6.20 & & & & 3.21 \\
$1723(1959)$ & $\tan \delta$ & $5.36 \times 10^{-3}$ & & & & \\
\hline
\end{tabular}


Table 27. Measurements on plastic specimens of polytetrafluoroethylene (PTFE), cross-linked polystyrene (CPS), fluoroethylenepropylene (FEP), polymethylmethacrylate (PMMA), and nylon. The standard type A expanded uncertainties, $k=2$, are as follows: parallel-plate dielectric resonator: $U_{\epsilon^{\prime}}=0.5 \%$, $U_{\tan \delta}=2 \times 10^{-5}, \mathrm{TE}_{01}$ cavity resonator: $U_{\epsilon^{\prime}}=1 \%, U_{\tan \delta}=1 \times 10^{-4}$, reentrant cavity: $U_{\epsilon^{\prime}}=2 \%, U_{\tan \delta}=2 \times 10^{-4}$.

\begin{tabular}{c|cccc}
\hline Material & Value & Parallel plate & $60 \mathrm{~mm} \mathrm{TE} \mathrm{m}_{01}$ & Reentrant cavity \\
\hline FEP & $\mathrm{f}(\mathrm{GHz})$ & 6.59 & 9.837 & \\
& $\epsilon^{\prime}$ & 2.064 & 2.025 & \\
& $\tan \delta$ & $7.04 \times 10^{-4}$ & $3.12 \times 10^{-4}$ & \\
\hline PTFE & $\mathrm{f}(\mathrm{GHz})$ & 9.93 & 9.816 & 1.082 \\
& $\epsilon^{\prime}$ & 2.05 & 2.055 & 2.06 \\
& $\tan \delta$ & $2.0 \times 10^{-4}$ & $2.1 \times 10^{-4}$ & $3 . \times 10^{-4}$ \\
\hline CPS & $\mathrm{f}(\mathrm{GHz})$ & 5.7 & 9.982 & 1.032 \\
& $\epsilon^{\prime}$ & 2.542 & 2.533 & 2.56 \\
& $\tan \delta$ & $5.1 \times 10^{-4}$ & $4.2 \times 10^{-4}$ & $7 \times 10^{-4}$ \\
\hline Nylon & $\mathrm{f}(\mathrm{GHz})$ & 4.75 & 9.742 & \\
& $\epsilon^{\prime}$ & 3.08 & 3.01 & \\
& $\tan \delta$ & $8.4 \times 10^{-3}$ & $7.1 \times 10^{-3}$ & \\
\hline PMMA & $\mathrm{f}(\mathrm{GHz})$ & 5.721 & 9.813 & \\
& $\epsilon^{\prime}$ & 2.634 & 2.626 & \\
& $\tan \delta$ & $7.2 \times 10^{-3}$ & $4.4 \times 10^{-3}$ & \\
\hline
\end{tabular}


Table 28. Measurements on cubic single crystals. The standard type A expanded uncertainties, $k=2$, are as follows: parallel-plate dielectric resonator: $U_{\epsilon^{\prime}}=0.5 \%$, $U_{\tan \delta}=2 \times 10^{-5}$, whispering gallery Mode resonator: $U_{\epsilon^{\prime}}=0.1 \%, U_{\tan \delta}=2 \times 10^{-5}$. Anisotropic materials were measured either by two dominant modes $\left(\mathrm{TE}_{011}\right.$ and $\mathrm{HE}_{111}$ ) using the parallel plate rod resonator or two families of whispering gallery modes.

\begin{tabular}{c|cc}
\hline Material & Value & Parallel Plate \\
\hline $\mathrm{LaAlO}_{3}$ & freq. (GHz) & 18.38 \\
(cubic) & $\epsilon^{\prime}$ & 23.99 \\
& $\tan \delta$ & $1.0 \times 10^{-5}$ \\
\hline $\mathrm{NdGaO}_{3}$ & $\mathrm{f}(\mathrm{GHz})$ & 18.49 \\
(cubic) & $\epsilon^{\prime}$ & 21.82 \\
& $\tan \delta$ & $1.07 \times 10^{-4}$ \\
\hline Zirconia & $\mathrm{f}(\mathrm{GHz})$ & 4.30 \\
(cubic) & $\epsilon^{\prime}$ & 27.8 \\
& $\tan \delta$ & $3.28 \times 10^{-3}$ \\
\hline $\mathrm{GGG}$ & $\mathrm{f}(\mathrm{GHz})$ & 15.7 \\
$($ cubic $)$ & $\epsilon^{\prime}$ & 12.43 \\
& $\tan \delta$ & $2.64 \times 10^{-3}$ \\
\hline $\mathrm{SrTiO} 3$ & $\mathrm{f}(\mathrm{GHz})$ & 7.20 \\
(cubic) & $\epsilon^{\prime}$ & 310 \\
& $\tan \delta$ & $4.01 \times 10^{-4}$ \\
\hline $\mathrm{MgO}$ & $\mathrm{f}(\mathrm{GHz})$ & 17.46 \\
$($ cubic) & $\epsilon^{\prime}$ & 9.70 \\
& $\tan \delta$ & $2.5 \times 10^{-6}$ \\
\hline
\end{tabular}


Table 29. Measurements on noncubic single crystals. The A expanded uncertainties, $k=2$, are as follows: parallel-plate dielectric resonator: $U_{\epsilon^{\prime}}=0.5, U_{\tan \delta}=2 \times 10^{-5}$, whispering gallery Mode resonator: $U_{\epsilon^{\prime}}=0.1 \%, U_{\tan \delta}=2 \times 10^{-5}$. Anisotropic materials were measured either by two dominant modes $\left(\mathrm{TE}_{0111}\right.$ and $\left.\mathrm{HE}_{111}\right)$ using the parallel plate rod resonator or two families of whispering gallery modes.

\begin{tabular}{c|ccc}
\hline Material & Value & Parallel plate & Whispering gallery \\
\hline $\mathrm{Al}_{2} \mathrm{O}_{3}$ & $\mathrm{f}(\mathrm{GHz})$ & 10.0 & 12.4 \\
$\|$ c-axis & $\epsilon^{\prime}$ & 11.58 & 11.58 \\
sapphire & $\tan \delta$ & $2 \times 10^{-5}$ & $5 . \times 10^{-6}$ \\
$\perp$ c-axis & $\epsilon^{\prime}$ & & 9.40 \\
& $\tan \delta$ & $2 \times 10^{-5}$ & $7 . \times 10^{-6}$ \\
\hline $\mathrm{LiNbO}_{3}$ & $\mathrm{f}(\mathrm{GHz})$ & 10.19 & \\
$\| \mathrm{c}$-axis & $\epsilon^{\prime}$ & 32.97 & \\
& $\tan \delta$ & $8 . \times 10^{-5}$ & \\
$\mathrm{LiNbO}_{3}$ & $\mathrm{f}(\mathrm{GHz})$ & 11.56 & \\
$\perp$ c-axis & $\epsilon^{\prime}$ & 42.34 & \\
& $\tan \delta$ & $8.4 \times 10^{-5}$ & \\
\hline Quartz & $\mathrm{f}(\mathrm{GHz})$ & 7.75 & \\
$\perp$ c-axis & $\epsilon^{\prime}$ & 4.59 & \\
& $\tan \delta$ & $1.5 \times 10^{-5}$ & \\
\hline Quartz & $\mathrm{f}(\mathrm{GHz})$ & 9.03 & \\
$\|$ c-axis & $\epsilon^{\prime}$ & 4.443 & \\
& $\tan \delta$ & $1.3 \times 10^{-5}$ & \\
\hline TiO & \\
$\|$ c-axis & $\mathrm{f}(\mathrm{GHz})$ & & 4.2 \\
& $\epsilon^{\prime}$ & & 83.7 \\
$\perp$ c-axis & $\tan \delta$ & & \\
& $\epsilon^{\prime}$ & & $8 \times 10^{-4}$ \\
\hline
\end{tabular}


Table 30. Measurements on low-medium permittivity TransTech ceramic specimens. The A expanded uncertainties, $k=2$, are as follows: parallel-plate dielectric resonator: $U_{\epsilon^{\prime}}=0.5 \%, U_{\tan \delta}=2 \times 10^{-5}, \mathrm{TE}_{01}$ cavity resonator: $U_{\epsilon^{\prime}}=1 \%$, $U_{\tan \delta}=1 \times 10^{-4}$, whispering gallery mode resonator: $U_{\epsilon^{\prime}}=0.1 \%, U_{\tan \delta}= \pm 2 \times 10^{-5}$.

\begin{tabular}{c|cccc}
\hline Material & Value & Parallel Plate & $60 \mathrm{~mm}$ Cavity & Whispering gallery \\
\hline Ceramic & $\mathrm{f}(\mathrm{GHz})$ & 3.675 & 9.76 & \\
4 & $\epsilon^{\prime}$ & 4.704 & 4.686 & \\
& $\tan \delta$ & $6.8 \times 10^{-4}$ & $5.80 \times 10^{-4}$ & \\
\hline Ceramic & $\mathrm{f}(\mathrm{GHz})$ & 3.17 & 9.74 & \\
6 & $\epsilon^{\prime}$ & 6.592 & 6.592 & \\
& $\tan \delta$ & $4.58 \times 10^{-4}$ & $5.30 \times 10^{-4}$ & \\
\hline Ceramic & $\mathrm{f}(\mathrm{GHz})$ & 2.713 & 9.74 & \\
9 & $\epsilon^{\prime}$ & 9.808 & 9.73 & 9.528 \\
& $\tan \delta$ & $2.55 \times 10^{-4}$ & $2.50 \times 10^{-4}$ & 9.98 \\
\hline Alumina & $\mathrm{f}(\mathrm{GHz})$ & 3.27 & 9.53 & $3.2 \times 10^{-5}$ \\
$(1986)$ & $\epsilon^{\prime}$ & 10.04 & 10.02 & \\
& $\tan \delta$ & $3.9 \times 10^{-5}$ & $3.38 \times 10^{-5}$ & \\
\hline Alumina & $\mathrm{f}(\mathrm{GHz})$ & 9.00 & & \\
$(1996)$ & $\epsilon^{\prime}$ & 9.992 & & \\
& $\tan \delta$ & $1.30 \times 10^{-3}$ & & \\
\hline
\end{tabular}

Additional measurements on plastics are given in Appendix B. In table 32 and figures 58,59 , and 60 , the permittivities of 7940 and 1723 Corning glass are displayed using various measurement fixtures.

Figures 62 through 67 and tables 33 through 35 tabulate measurements of a number of ceramics, polymers, and PWB laminate materials made by other researchers. 
Table 31. Measurements on high-permittivity TransTech ceramic specimens. The A expanded uncertainties, $k=2$, for the parallel-plate dielectric resonator: $U_{\epsilon^{\prime}}=0.5$ $\%, U_{\tan \delta}=2 \times 10^{-5}, \mathrm{TE}_{01}$ cavity resonator: $U_{\epsilon^{\prime}}=1 \%, U_{\tan \delta}=1 \times 10^{-4}$, whispering gallery mode resonator: $U_{\epsilon^{\prime}}=0.1 \%, U_{\tan \delta}= \pm 2 \times 10^{-5}$

\begin{tabular}{|c|c|c|c|c|}
\hline Material & Value & Parallel plate & $60 \mathrm{~mm}$ cavity & Whispering gallery \\
\hline Ceramic & $\mathrm{f}(\mathrm{GHz})$ & 1.987 & 9.72 & 9.577 \\
\hline \multirow[t]{2}{*}{13} & $\epsilon^{\prime}$ & 13.51 & 13.55 & 13.54 \\
\hline & $\tan \delta$ & $1.07 \times 10^{-4}$ & $1.10 \times 10^{-4}$ & $1.01 \times 10^{-4}$ \\
\hline Ceramic & $\mathrm{f}(\mathrm{GHz})$ & 1.834 & 9.71 & 9.692 \\
\hline \multirow[t]{2}{*}{16} & $\epsilon^{\prime}$ & 16.60 & 16.64 & 16.66 \\
\hline & $\tan \delta$ & $2.43 \times 10^{-5}$ & $0.4 \times 10^{-4}$ & $5.70 \times 10^{-5}$ \\
\hline Ceramic & $\mathrm{f}(\mathrm{GHz})$ & 1,617 & 9.70 & \\
\hline \multirow[t]{2}{*}{20} & $\epsilon^{\prime}$ & 20.77 & 20.80 & \\
\hline & $\tan \delta$ & $8.4 \times 10^{-5}$ & $2.00 \times 10^{-4}$ & \\
\hline Ceramic & $\mathrm{f}(\mathrm{GHz})$ & 1.328 & 9.44 & 6.023 \\
\hline \multirow[t]{2}{*}{31} & $\epsilon^{\prime}$ & 30.96 & 31.03 & 30.99 \\
\hline & $\tan \delta$ & $2.4 \times 10^{-5}$ & $5.0 \times 10^{-5}$ & $4.9 \times 10^{-5}$ \\
\hline Ceramic & $\mathrm{f}(\mathrm{GHz})$ & 1.200 & 9.46 & 5.460 \\
\hline \multirow[t]{2}{*}{35} & $\epsilon^{\prime}$ & 35.15 & 35.19 & 35.14 \\
\hline & $\tan \delta$ & $4.8 \times 10^{-5}$ & $1.6 \times 10^{-4}$ & $1.18 \times 10^{-4}$ \\
\hline Ceramic & $\mathrm{f}(\mathrm{GHz})$ & 1.116 & 9.46 & 4.812 \\
\hline \multirow[t]{2}{*}{36} & $\epsilon^{\prime}$ & 36.55 & 36.56 & 36.55 \\
\hline & $\tan \delta$ & $3.3 \times 10^{-5}$ & $1.2 \times 10^{-4}$ & $8.8 \times 10^{-5}$ \\
\hline Ceramic & $\mathrm{f}(\mathrm{GHz})$ & 1.109 & 9.73 & \\
\hline \multirow[t]{2}{*}{38} & $\epsilon^{\prime}$ & 38.65 & 38.77 & \\
\hline & $\tan \delta$ & $5.8 \times 10^{-5}$ & $3.0 \times 10^{-4}$ & \\
\hline Ceramic & $\mathrm{f}(\mathrm{GHz})$ & 2.83 & & \\
\hline \multirow[t]{2}{*}{78} & $\epsilon^{\prime}$ & 78.29 & & \\
\hline & $\tan \delta$ & $3.1 \times 10^{-4}$ & & \\
\hline Ceramic & $\mathrm{f}(\mathrm{GHz})$ & 0.855 & 9.44 & 4.448 \\
\hline \multirow[t]{2}{*}{79} & $\epsilon^{\prime}$ & 79.27 & 79.29 & 79.11 \\
\hline & $\tan \delta$ & $1.63 \times 10^{-4}$ & $7.8 \times 10^{-4}$ & $4.40 \times 10^{-4}$ \\
\hline
\end{tabular}




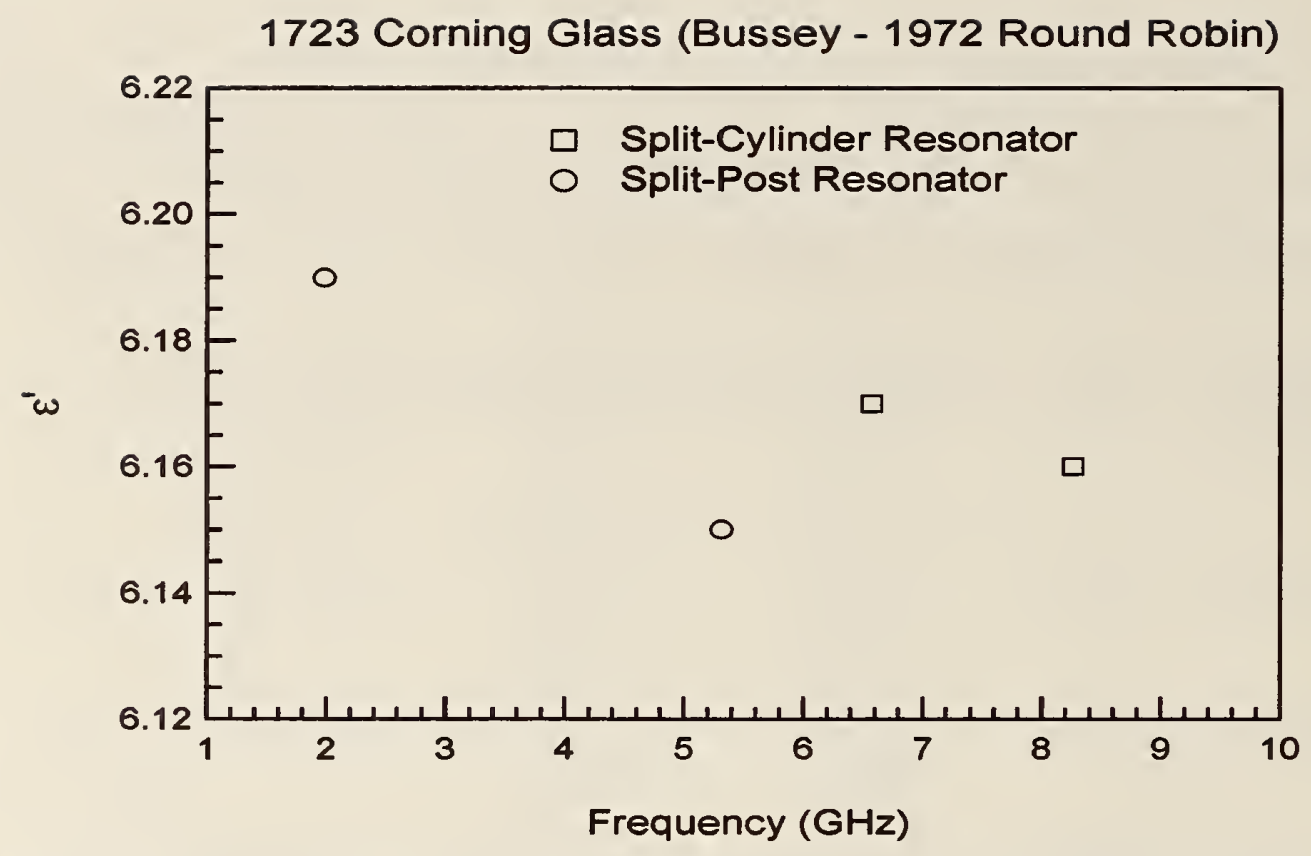

Figure 58. Measurements of the real part of the permittivity of Corning 1723 glass.

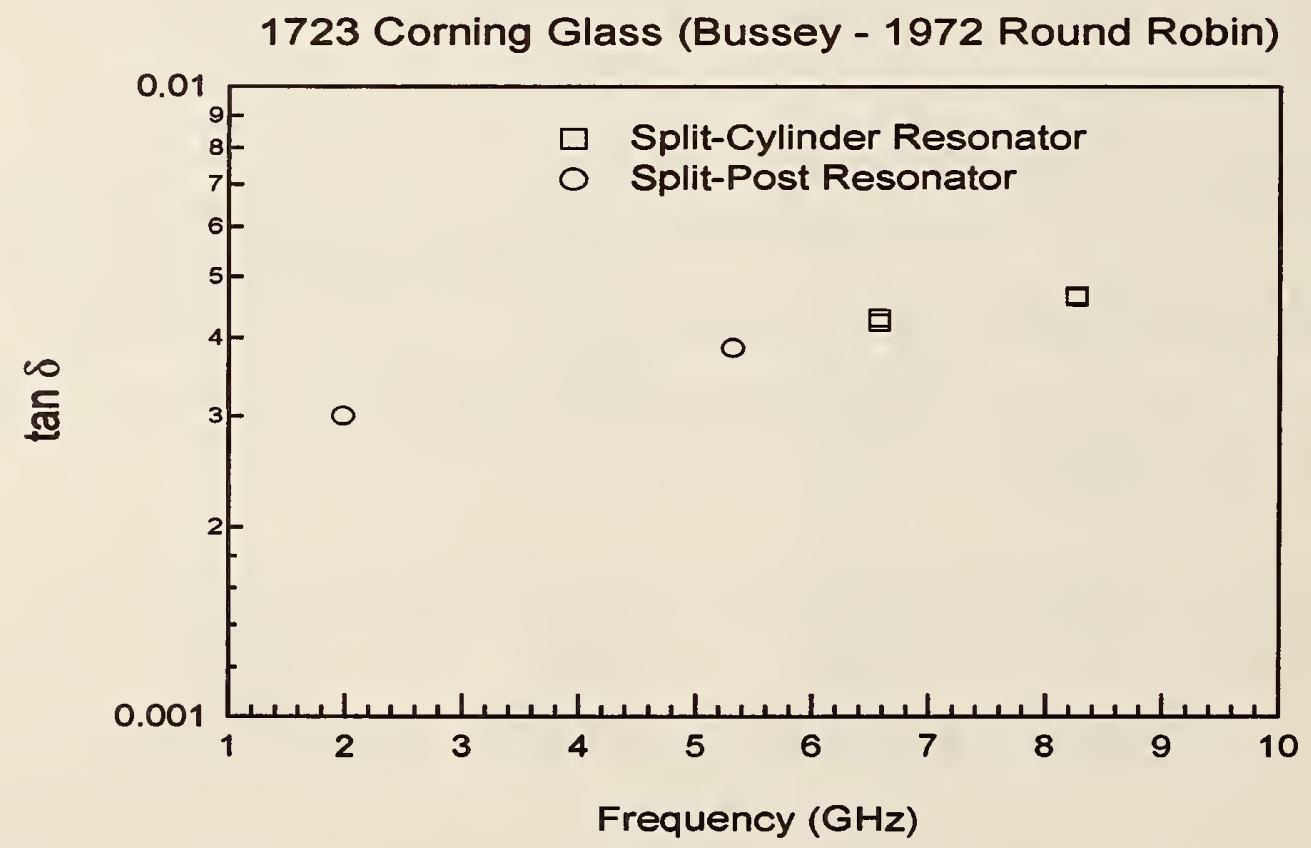

Figure 59. Measurements of the loss tangent of Corning 1723 glass. 
Table 32. The 1964 round-robin measurements on Corning 1723 and 7940 glasses compared with recent measurements [151]. The round-robin results from 1964 are given below $[152,153]$. The $\mathrm{A}$ expanded uncertainties, $k=2$, are as follows: parallelplate dielectric resonator: $U_{\epsilon^{\prime}}=0.5 \%, U_{\tan \delta}=2 \times 10^{-4}, \mathrm{TE}_{01}$ cavity resonator: $U_{\epsilon^{\prime}}=1 \%, U_{\tan \delta}=1 \times 10^{-4}$.

\begin{tabular}{c|ccccc}
\hline Material & Value & NBS(1964) & Parallel Plate & $\mathrm{TE}_{01}(50 \mathrm{~mm})$ & $\mathrm{TE}_{01}(60 \mathrm{~mm})$ \\
\hline 1723 Glass & $\mathrm{f}(\mathrm{GHz})$ & 9 & & 10 & \\
$(1964 \mathrm{RR})$ & $\epsilon^{\prime}$ & 6.20 & & 6.26 & \\
& $\tan \delta$ & $5.3 \times 10^{-3}$ & & $4.8 \times 10^{-3}$ & \\
\hline $1723 \mathrm{Glass}$ & $\mathrm{f}(\mathrm{GHz})$ & 9 & & 9.58 & 10 \\
$(1972 \mathrm{RR})$ & $\epsilon^{\prime}$ & 6.155 & & 6.16 & 6.146 \\
& $\tan \delta$ & $4.7 \times 10^{-3}$ & & $4.9 \times 10^{-3}$ & $4.7 \times 10^{-3}$ \\
\hline $7940 \mathrm{Glass}$ & $\mathrm{f}(\mathrm{GHz})$ & 9 & 9 & 10 & 9.82 \\
$(1972 \mathrm{RR})$ & $\epsilon^{\prime}$ & 3.826 & 3.822 & 3.828 & 3.829 \\
& $\tan \delta$ & $1.2 \times 10^{-4}$ & $1.4 \times 10^{-4}$ & $1.03 \times 10^{-4}$ & $1.2 \times 10^{-4}$ \\
\hline
\end{tabular}

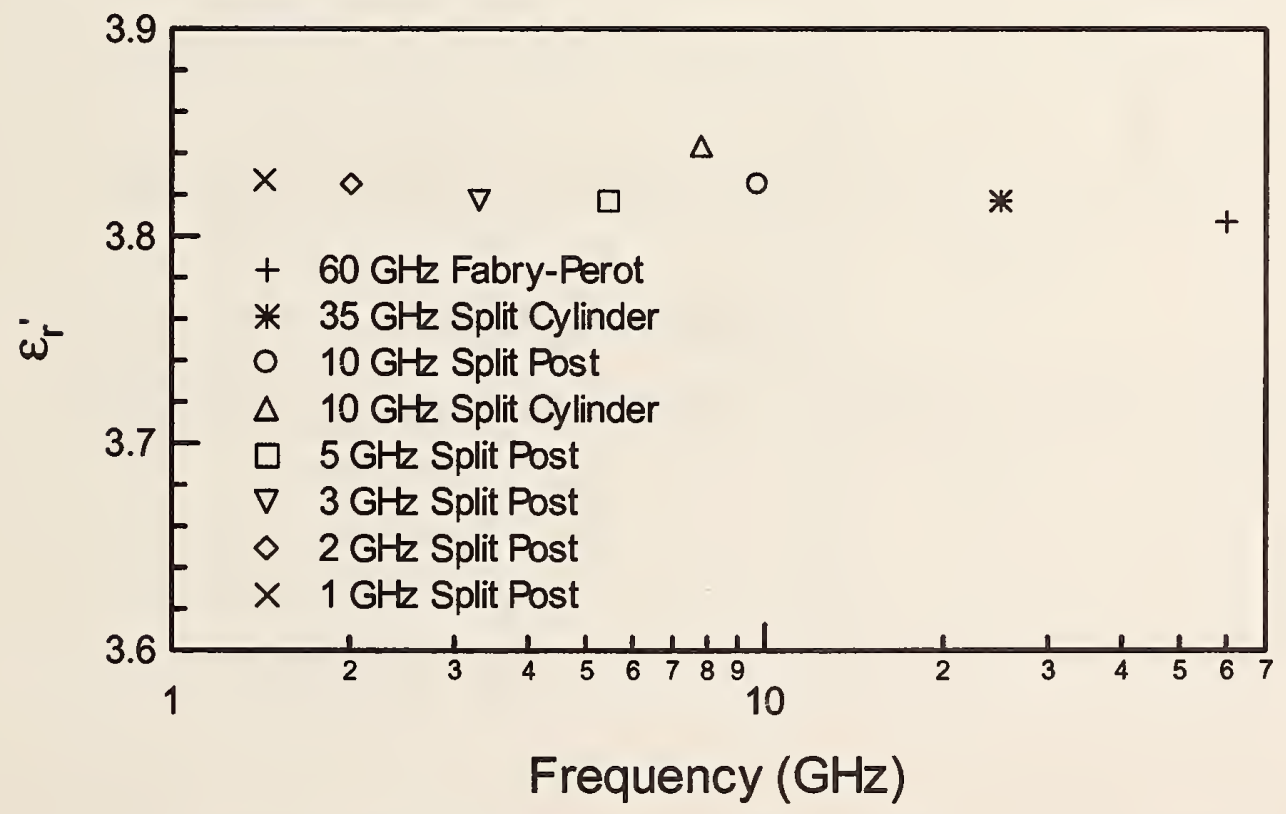

Figure 60. Measurements on the real part of the permittivity of Corning 7940 glass. 


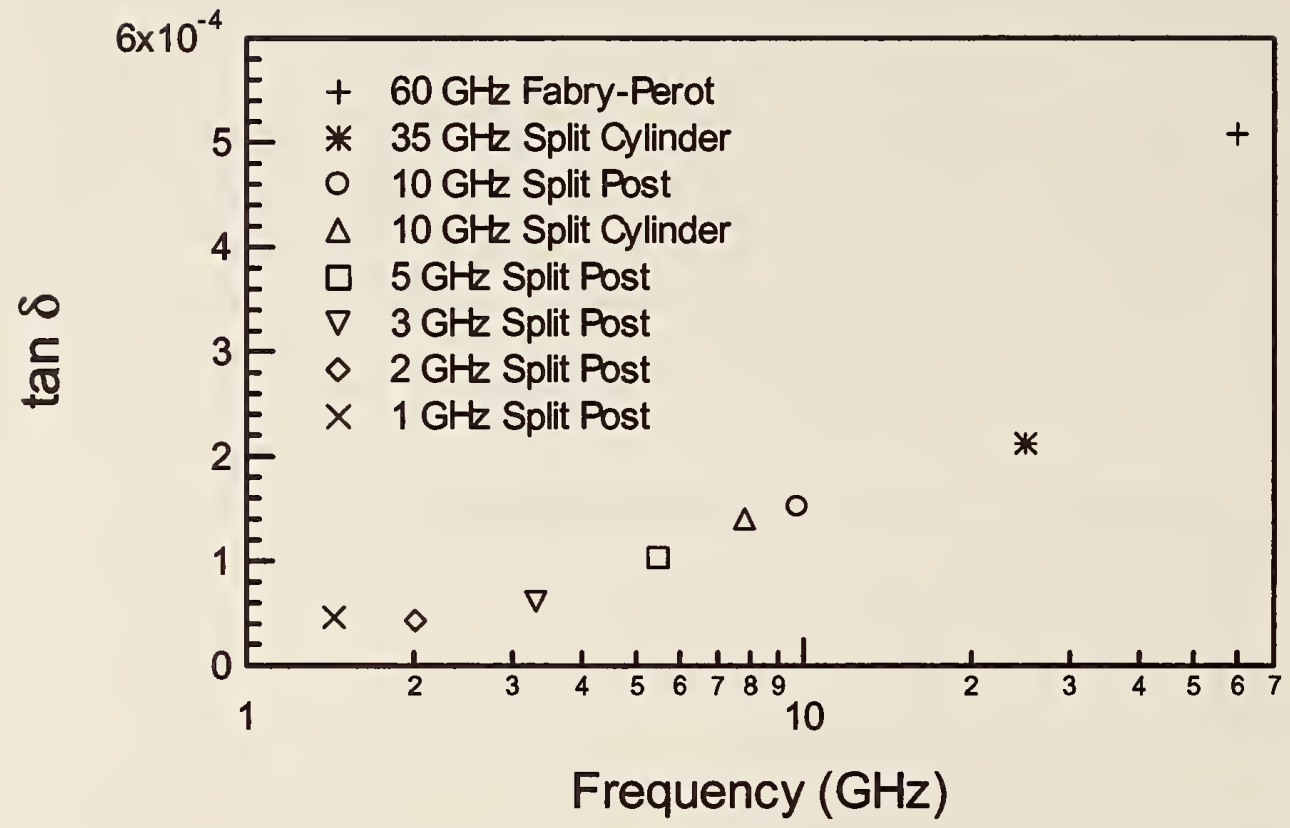

Figure 61. Measurements of the loss tangent of Corning 7940 glass. 
Table 33. Typical dielectric measurements on materials (no uncertainties assigned).

\begin{tabular}{c|cccc}
\hline Material & Crystal & $\mathrm{f}(\mathrm{GHz})$ & $\epsilon_{r}^{\prime}$ & $\tan \delta$ \\
\hline Aluminum nitride [154] & No & 8.5 & 8.5 & 0.0030 \\
Barium borate & Yes & 0.001 & 4.6 & 0.0005 \\
Beryllium oxide [154] & Yes & 8.5 & 6.86 & 0.0003 \\
Boron nitride [154] & No & 5.0 & 4.30 & 0.0002 \\
Calcium carbonate [154] & Yes & 0.0001 & 8.5 & 0.0004 \\
Calcium fluoride [154] & Yes & 1 & 6.3 & 0.0006 \\
Germanium [154] & Yes & 10 & 16.6 & \\
Lithium niobate [154] & Yes & 10 & 29 & \\
Neodymium gallate [154] & Yes & 10 & 20 & 0.0030 \\
Nickel oxide [154] & No & 1 & 7.5 & 0.0001 \\
Silicon nitride [154] & No & 8.5 & 5.5 & 0.0036 \\
Potassium bromide [154] & Yes & 10 & 4.9 & \\
Silicon [154] & Yes & 14 & 12 & 0.0090 \\
Silicon carbide [155] & No & 10 & 47 & 0.0010 \\
Sodium chloride [154] & Yes & 0.001 & 5.7 & 0.0001 \\
Strontium lanthanum aluminate [154] & Yes & 10 & 17 & 0.0008 \\
Strontium lanthanum gallate [154] & Yes & 10 & 22 & 0.0001 \\
Topaz [154] & Yes & 0.01 & 6.5 & 0.0001 \\
Yttrium iron garnet [154] & Yes & 10 & 14.9 & 0.0001 \\
Yttrium oxide [154] & No & 0.001 & 11.3 & 0.0006 \\
Zinc oxide [154] & No & 0.01 & 8.3 & 0.0300
\end{tabular}


Table 34. Dielectric measurements on polymers (no uncertainties assigned).

\begin{tabular}{c|ccc}
\hline Material & $\mathrm{f}(\mathrm{GHz})$ & $\epsilon_{r}^{\prime}$ & $\tan \delta$ \\
\hline PMMA [154] & 3.0 & 2.61 & 0.0057 \\
Nylon [154] & 3.0 & 2.84 & 0.0117 \\
Polyethylene [154] & 3.0 & 2.26 & 0.0003 \\
Styrofoam [154] & 3.0 & 1.03 & 0.0002 \\
Polyimide [148] & 0.001 & 3.50 & 0.003 \\
BPDA-PDA Polyimide [148] & 0.001 & 3.8 & \\
Polysulfone [156] & 1.0 & 3.02 & 0.0090 \\
Polyolefin [156] & 1.0 & 2.75 & 0.0200 \\
Cyanate Ester [148] & 0.001 & 3.48 & 0.01 \\
\hline
\end{tabular}


Table 35. Dielectric measurements of PWB composites (no uncertainties assigned).

\begin{tabular}{cc}
\hline Material & Range of $\epsilon_{r}^{\prime}$ \\
\hline FR-4 [140] & $4.2-5.0$ \\
FR-5 (high temp) [140] & $4.3-4.6$ \\
BT/epoxy [140] & $4.0-4.2$ \\
Cyanate ester [140] & $3.5-3.7$ \\
Arimid/epoxy [140] & $3.7-3.9$ \\
Polyimide/glass [140] & $3.9-4.2$ \\
Polyimide/quartz [140] & $3.4-3.5$ \\
\hline
\end{tabular}




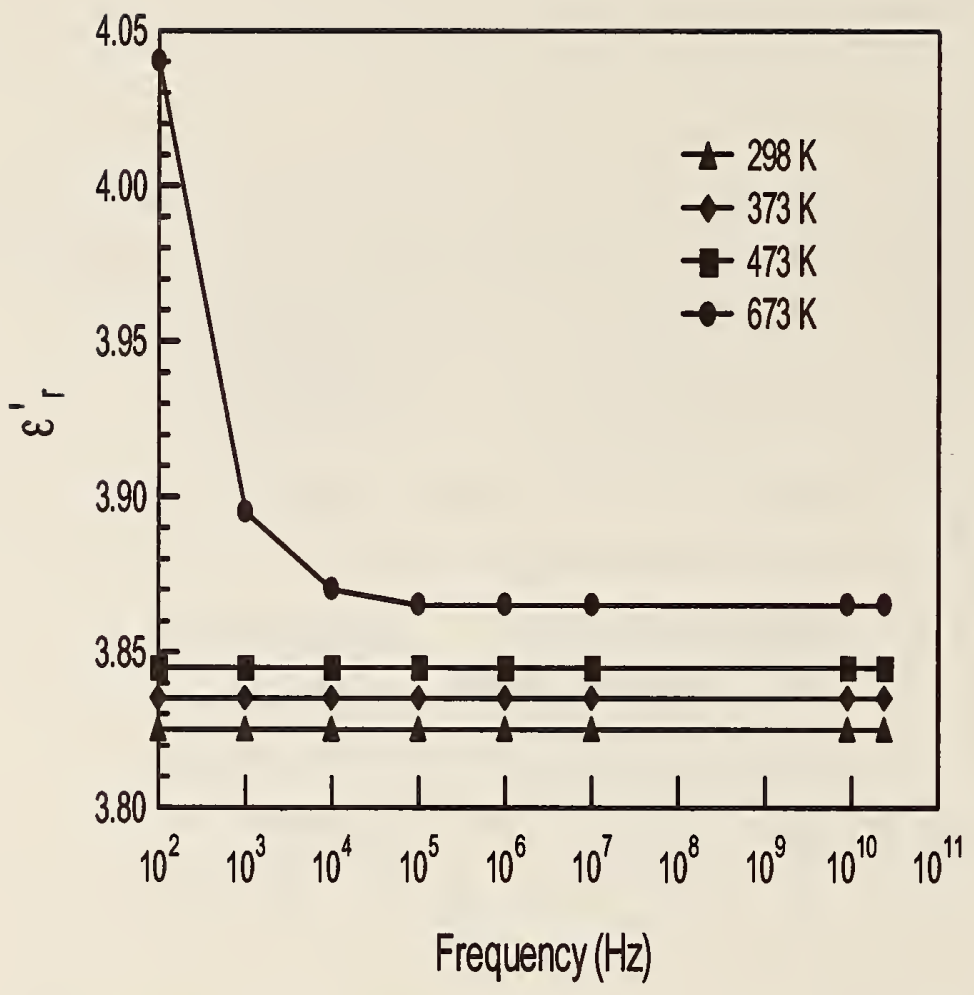

Figure 62. Measurements of $\epsilon_{r}^{\prime}$ of $\mathrm{SiO}_{2}$ as a function of temperature [154] (no uncertainties assigned). 


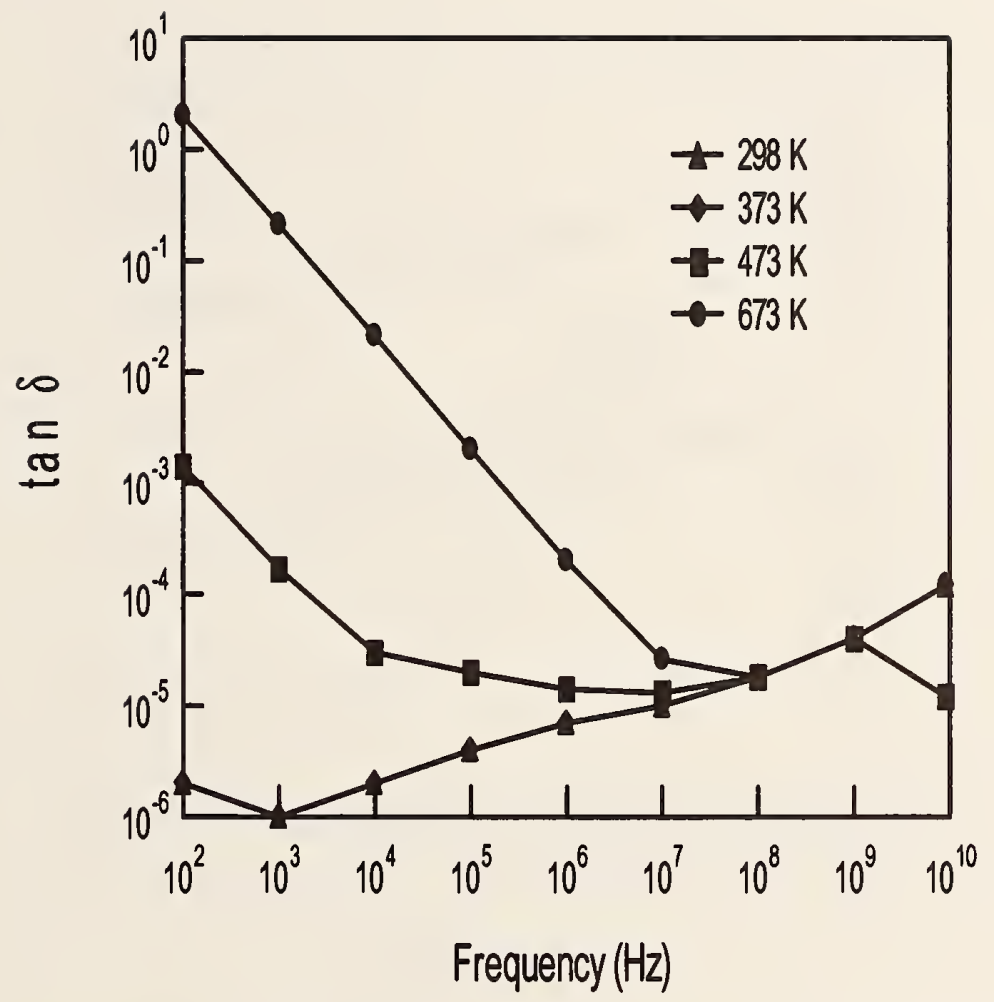

Figure 63. Measurements of the loss tangent part of $\mathrm{SiO}_{2}$ as a function of temperature [154] (no uncertainties assigned). 


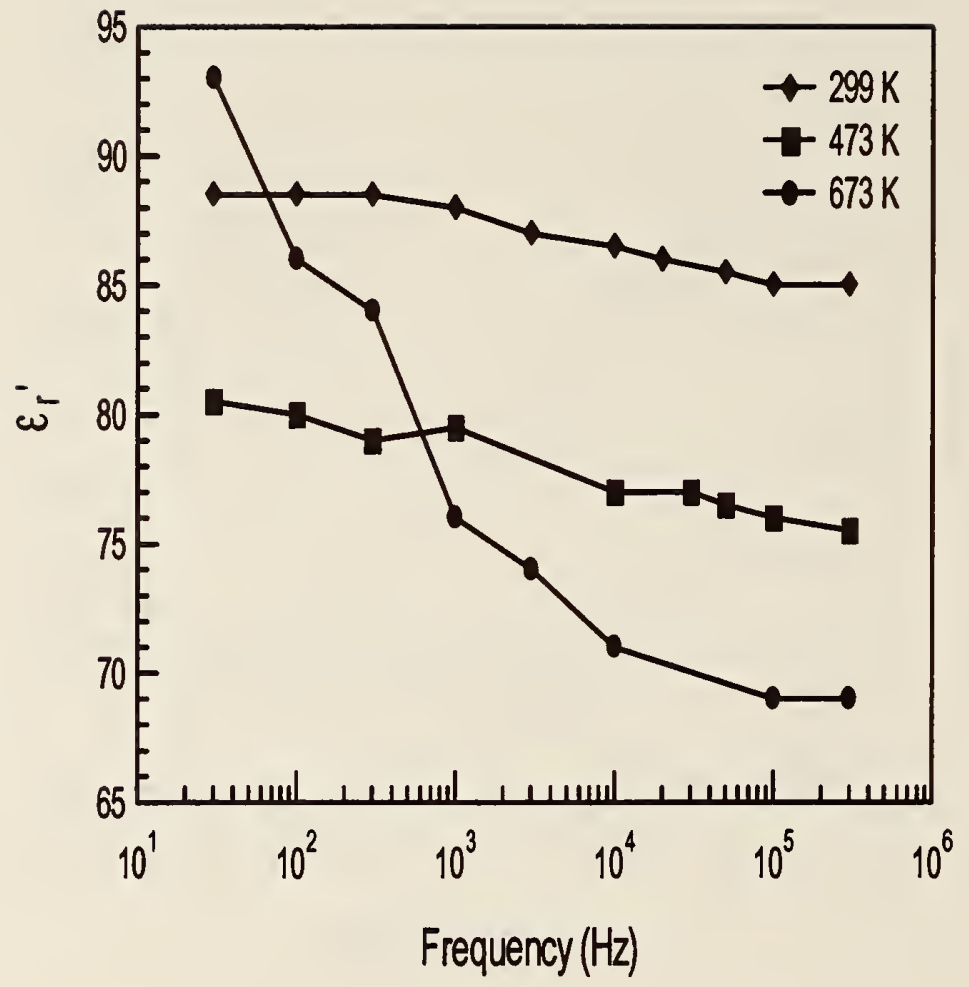

Figure 64. Measurements of $\epsilon_{r}^{\prime}$ for rutile with electric field perpendicular to axis as a function of temperature [154] (no uncertainties assigned). 


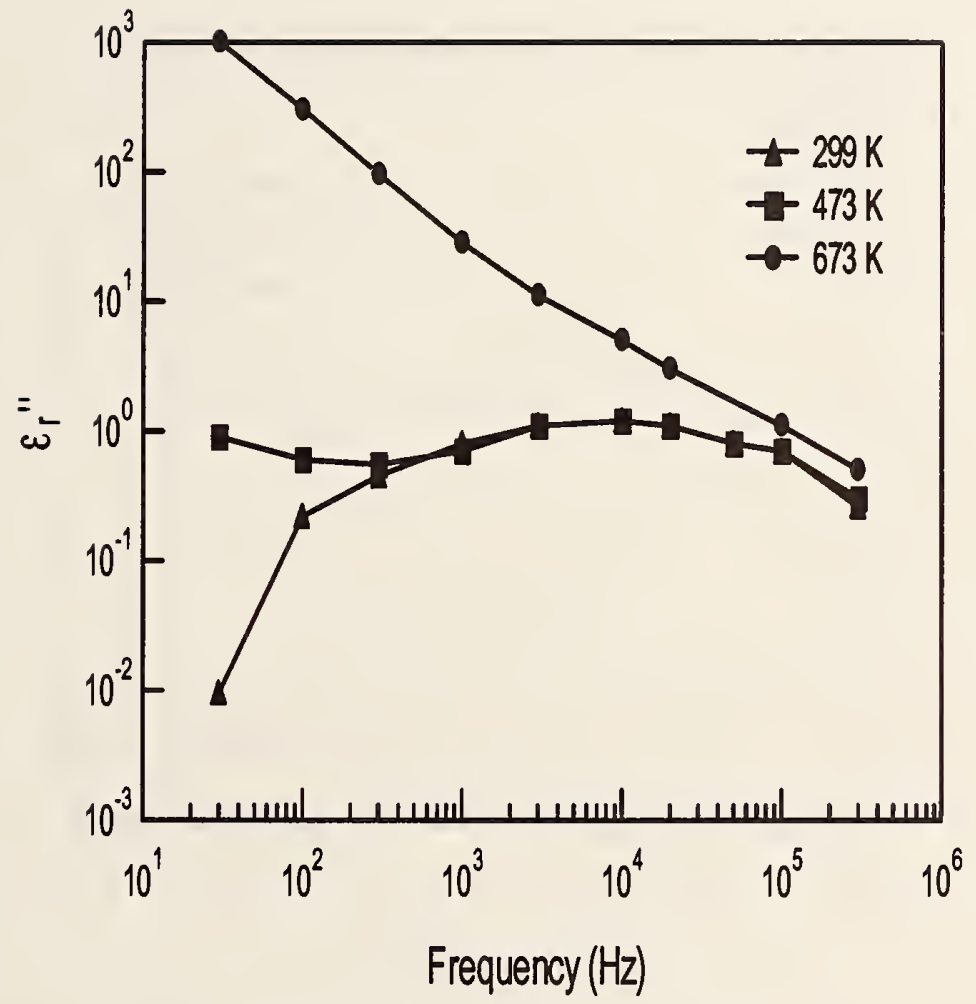

Figure 65. Measurements of $\epsilon_{r}^{\prime \prime}$ for rutile with electric field perpendicular to axis as a function of temperature [154] (no uncertainties assigned). 


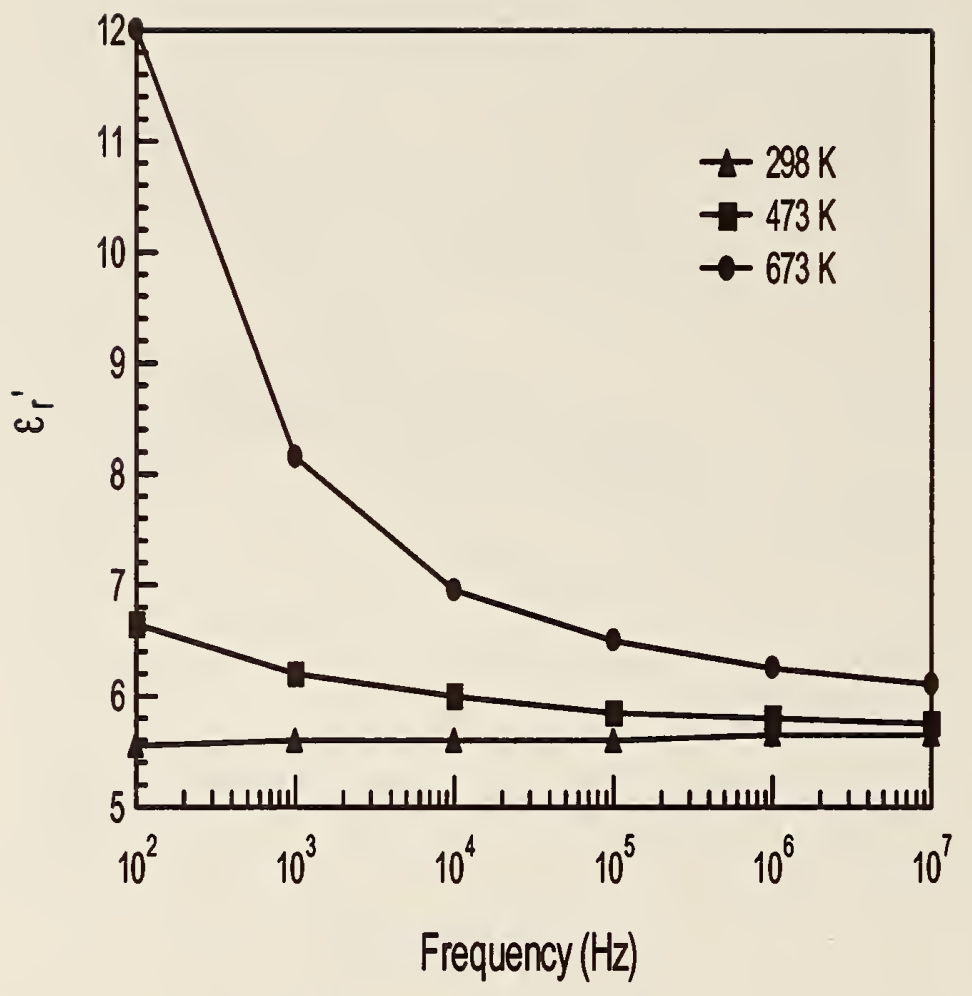

Figure 66. Measurements on $\epsilon_{r}^{\prime}$ for silicon nitride as a function of temperature [154] (no uncertainties assigned). 


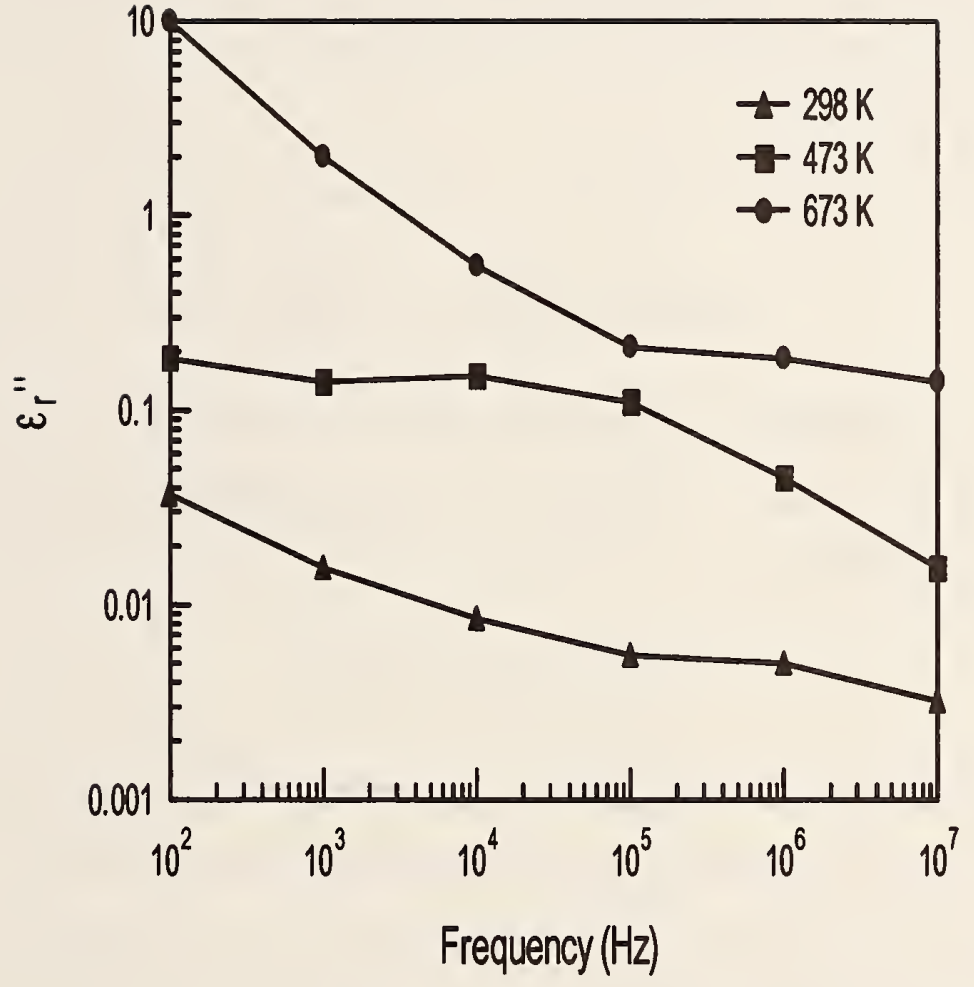

Figure 67. Measurements on $\epsilon_{r}^{\prime \prime}$ for silicon nitride as a function of temperature [154] (no uncertainties assigned). 


\section{Elevated Temperature Measurements}

\subsection{Thermal Properties}

When measuring permittivity as a function of temperature, it is important to use accurate thermal expansion data at each temperature. The two thermal properties relevant to substrates for electronic packaging are the coefficient of thermal conductivity $(k)$ and coefficient of thermal expansion $\left(\alpha_{\text {CTE }}\right)$. The CTE of many polymers is strongly temperature dependent and care must be taken to account for this variable in a packaged circuit.

The coefficient of thermal expansion $\alpha_{\mathrm{CTE}}$ is defined as

$$
\alpha_{\mathrm{CTE}}=\frac{1}{L}\left[\frac{\partial L}{\partial T}\right]_{P} \approx \frac{1}{L} \frac{\Delta L}{\Delta T},
$$

where $L$ is material thickness, $P$ is pressure, and $\Delta L$ is change in thickness as temperature changes by $\Delta T$. For temperature-dependent thermal expansion coefficients we can integrate eq (97) to get

$$
L=L_{0} \exp \left(\int_{T_{0}}^{T} \alpha(T) d T\right) .
$$

Typical coefficients of thermal expansion are given for a number of materials in tables 36 and $37[1,17,155,158]$. If the temperature is not specified, then it is for 295 $\mathrm{K}$. These results were taken from various sources and in some cases from company specification sheets [159]. Polymers can have large temperature-dependent CTE and low glass transition temperatures $T_{g}$ (see table 38 ). The glass transition temperature is where a polymer starts to change phase to a more liquid-like state.

The temperature coefficient of permittivity is defined as

$$
\tau_{\epsilon}=\frac{1}{\epsilon_{r}^{\prime}}\left[\frac{\partial \epsilon_{r}^{\prime}}{\partial T}\right]_{P} \approx \frac{1}{\epsilon_{r}^{\prime}} \frac{\Delta \epsilon_{r}^{\prime}}{\Delta T} .
$$

$\tau_{\epsilon}$ is usually expressed as parts in $10^{6}$ [52]. Materials with negative temperature coefficients can be combined with materials with positive temperature coefficients to 
Table 36. Typical coefficients of thermal expansion (CTE)(no uncertainties assigned). Temperature $295 K$ unless otherwise specified.

\begin{tabular}{cc}
\hline Material & $\alpha \times 10^{6}\left({ }^{\circ} \mathrm{C}^{-1}\right)$ \\
\hline ABS plastic & 70 \\
acetal & 85 \\
acrylic & 68 \\
alumina [1] & 6.7 \\
aluminum [1] & 24 \\
aluminum nitride [1] & 3.0 \\
aluminum oxide [1] & 2.6 \\
aluminum titanate & 0.4 \\
aramid/epoxy [140] & $6-9$ \\
beryllium oxide [1] & 6.5 \\
bismalimide triazine/epoxy [140] & $15-17$ \\
borosilicate glass [155] & 3.1 \\
calcium oxide & 14.8 \\
copper [1] & 17 \\
cordierite [1] & 1.0 \\
cyanate ester [140] & 13 \\
diamond [1] & 1.02 \\
epoxy [157] & 54 \\
FR-4 [1] & $17-18$ \\
FR-5 [1] & $17-18$ \\
fused quartz & 0.5 \\
fused SiO & $\mathrm{K}), 0.5(300 \mathrm{~K})$ \\
fused silica [1] & 0.5 \\
gallium arsenide [6] & 5.7 \\
gallium gadolinium garnet & 8.0 \\
germanium & 6.1 \\
kovar & 5.0 \\
\hline
\end{tabular}


Table 37. Typical coefficients of thermal expansion (continued) (CTE)(no uncertainties assigned). Temperature $295 K$ unless otherwise specified.

\begin{tabular}{cc}
\hline Material & $\alpha \times 10^{6}\left({ }^{\circ} \mathrm{C}^{-1}\right)$ \\
\hline lanthanum aluminate & 9.0 \\
lithium niobate & $15,4.1 \|$ \\
magnesium oxide (MGO) [1] & 8.0 \\
neodymium gallate & 9.0 \\
nylon & $50(193 \mathrm{~K}), 93(300 \mathrm{~K}), 146(400 \mathrm{~K})$ \\
PMMA & $11(40 \mathrm{~K}), 61(300 \mathrm{~K}), 172(450 \mathrm{~K})$ \\
polycarbonate & 66 \\
polyethylene (high density) & $33(50 \mathrm{~K})), 175(300 \mathrm{~K}), 940(400 \mathrm{~K})$ \\
polyimide [155] & 40 \\
polyimide/glass [1] & $14-15$ \\
polyimide/quartz [140] & $6-8$ \\
polypropylene [157] & $76(180 \mathrm{~K}), 94(250 \mathrm{~K}), 205(293 \mathrm{~K})$ \\
quartz (crystalline) [1] & 11.2 \\
Rexolite [6] & $26(30 \mathrm{~K}), 71(300 \mathrm{~K})$ \\
rutile [1] & 7.0 \\
sapphire [1] & 5.3 \\
silicon [1] & 3.0 \\
silicon carbide [1] & 3.7 \\
spinel [1] & 6.6 \\
strontium titanate & 9.0 \\
strontium lanthanum aluminate [6] & 7.0 \\
strontium lanthanum gallate [6] & 10 \\
Teflon [6] & $30(30 \mathrm{~K}), 124(330 \mathrm{~K}), 258(500 \mathrm{~K})$ \\
tridymite [1] & 17.5 \\
zirconia oxide & 7.5 \\
Zr-Sn titanate [1] & -6.9 \\
\hline &
\end{tabular}


Table 38. Typical glass transition temperature [140].

\begin{tabular}{cc} 
Material & $T_{g}\left({ }^{\circ} \mathrm{C}\right)$ \\
\hline Polyimide/quartz & 250 \\
Cyanate ester & 250 \\
Polyimide/glass & 250 \\
Teflon & 200 \\
BT/epoxy & 190 \\
FR-5 & 170 \\
Rexolite & 120 \\
FR-4 & 130 \\
\hline
\end{tabular}

produce a material with a small composite change with temperature; however the mixture properties need to be well-characterized.

The thermal coefficients of expansion for composites can be calculated by mixture formulas such as

$$
\alpha_{\mathrm{eff}}=\frac{\sum_{i} \alpha_{i} k_{i} \theta_{i}}{\sum_{i} k_{i} \theta_{i}}
$$

where $k_{i}, \alpha_{i}$, and $\theta_{i}$ are the bulk modulus, coefficient of expansion, and volume fraction of the ith phase [160].

One method for quantifying the effects of temperature on resonant frequency is the temperature coefficient of resonance frequency

$$
\tau_{f}=\frac{1}{f}\left[\frac{\partial f}{\partial T}\right]_{P} \approx \frac{1}{f} \frac{\Delta f}{\Delta T}
$$

Many ceramics have $\tau_{f}$ that are well-represented by a quadratic polynomial in temperature.

In addition to thermal expansion there is the problem of thermal warping of substrates. Warping or camber is a result of differential thermal expansion. A 
measure of camber can be estimated by

$$
\frac{\Delta W}{L}=\frac{\alpha_{w} L \Delta T}{d},
$$

where $\Delta W$ is the height of the warp of the material from a plane surface, $\alpha_{w}$ is coefficient of thermal bending, $L$ is the length of material, and $d$ is thickness [161].

\subsection{Elevated Temperature Measurement Results}

Many substrate and PWB permittivities vary strongly with temperature. In this section, we describe a system for making measurements at elevated temperature. We also present permittivity measurements as a function of temperature for a number of substrate materials. For these measurements we used a $\mathrm{TE}_{01 \delta}$ dielectric resonator as shown in figure 68 . In order to minimize wall losses, the specimen is supported by a low-loss dielectric rod. The temperature system, as shown in figure 69 , is an environmental chamber that operates from $-150^{\circ} \mathrm{C}$ to $150^{\circ} \mathrm{C}$. In this figure, a coaxial line system is shown; however, for the measurements in this report we used a resonant device. We can use cavities, transmission lines, and reentrant cavities in the chamber. The chamber has bulkhead adapters on the sides for insertion of coaxial feeds for the cavities or transmission lines. The chamber can also be purged with nitrogen gas to reduce oxidation and water vapor.

Measurements of some common low-permittivity materials are given in figures 70 to 75 . 


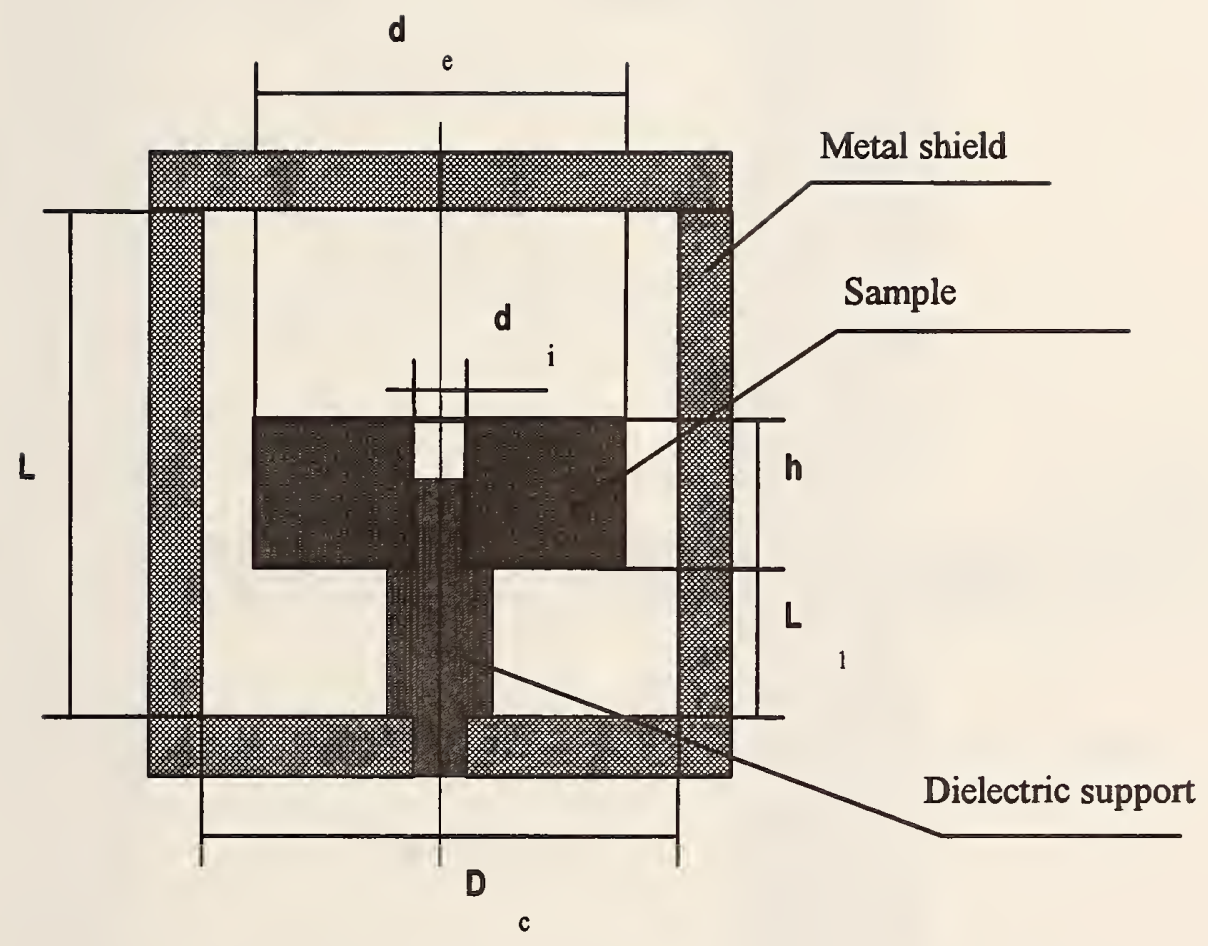

Figure $68 . \mathrm{TE}_{01 \delta}$ resonator for measurements at elevated temperatures. 


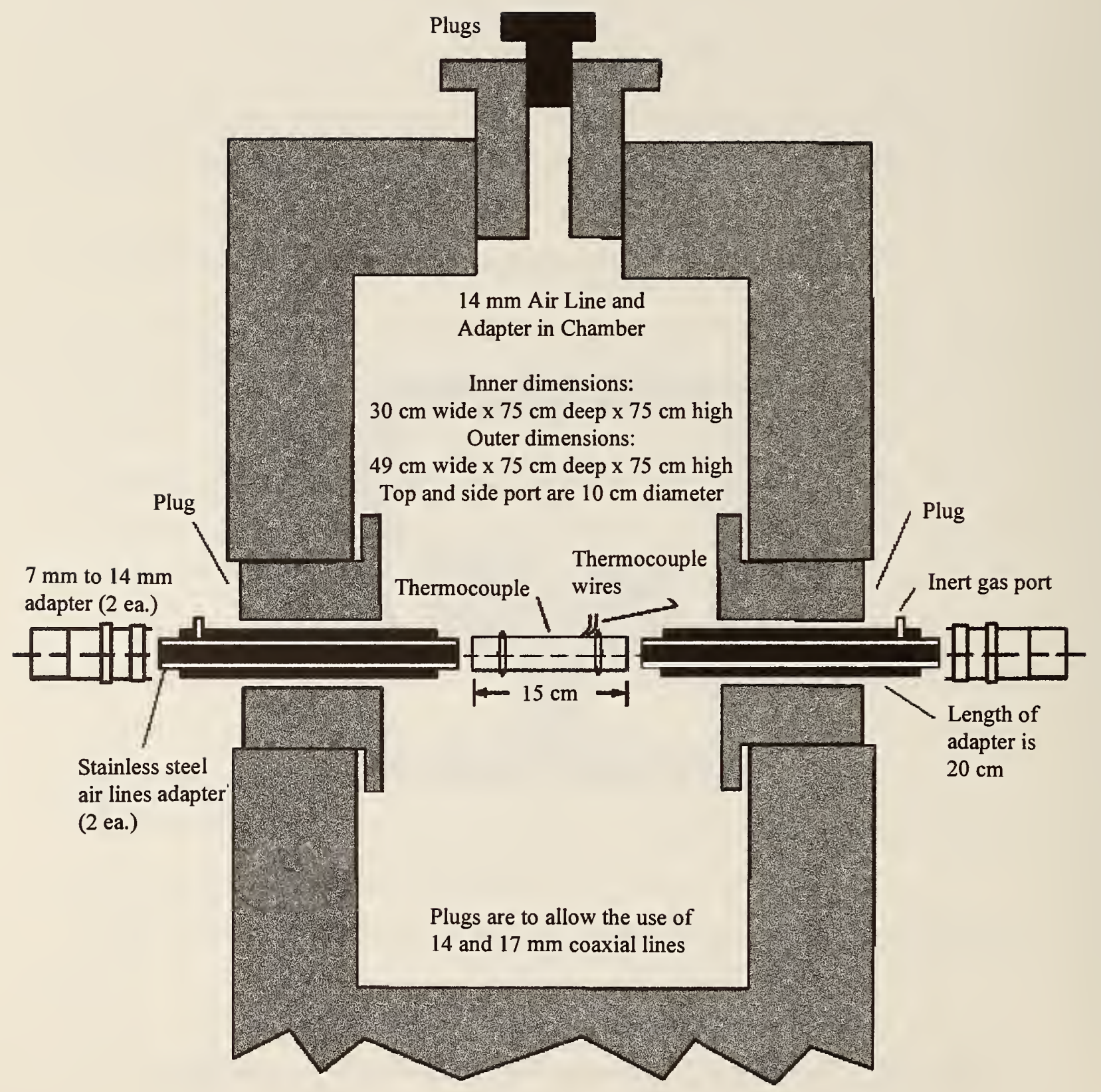

Figure 69. Environmental chamber for measurements at elevated temperatures. 


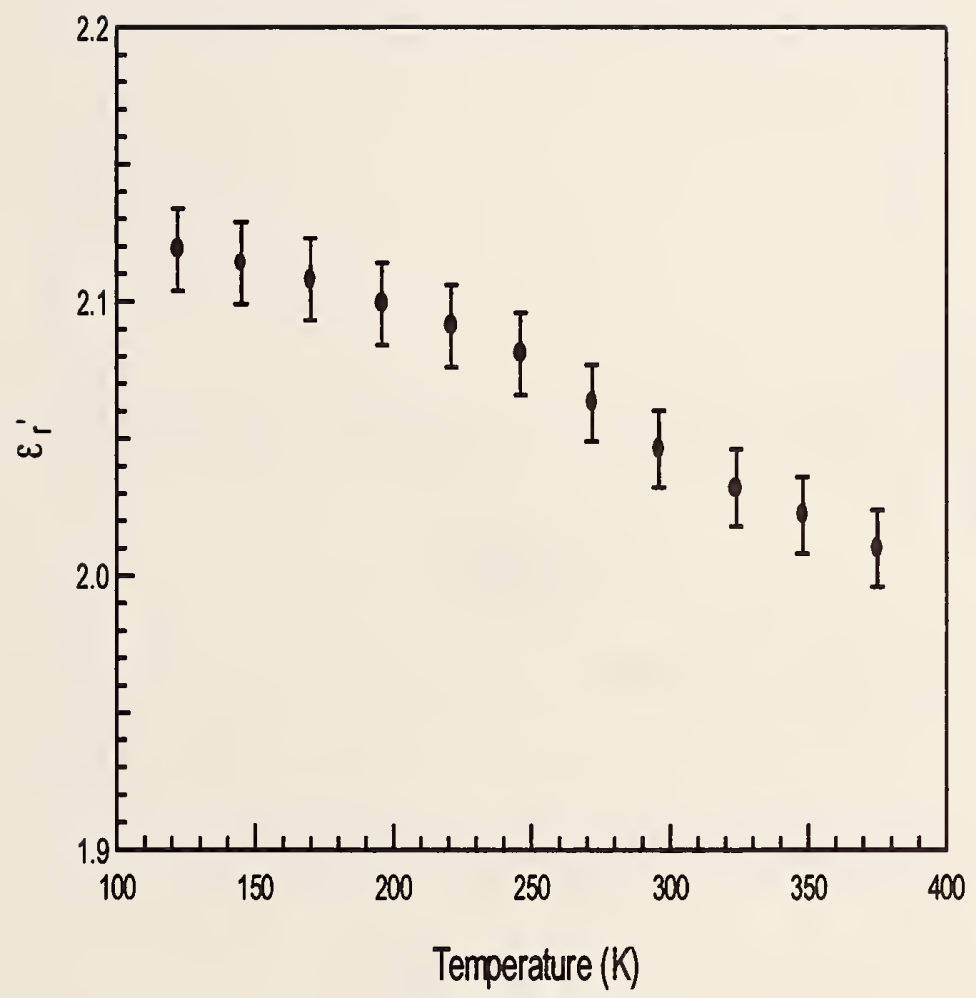

Figure 70. Real part of the permittivity of Teflon as a function of temperature, $k=2$. 


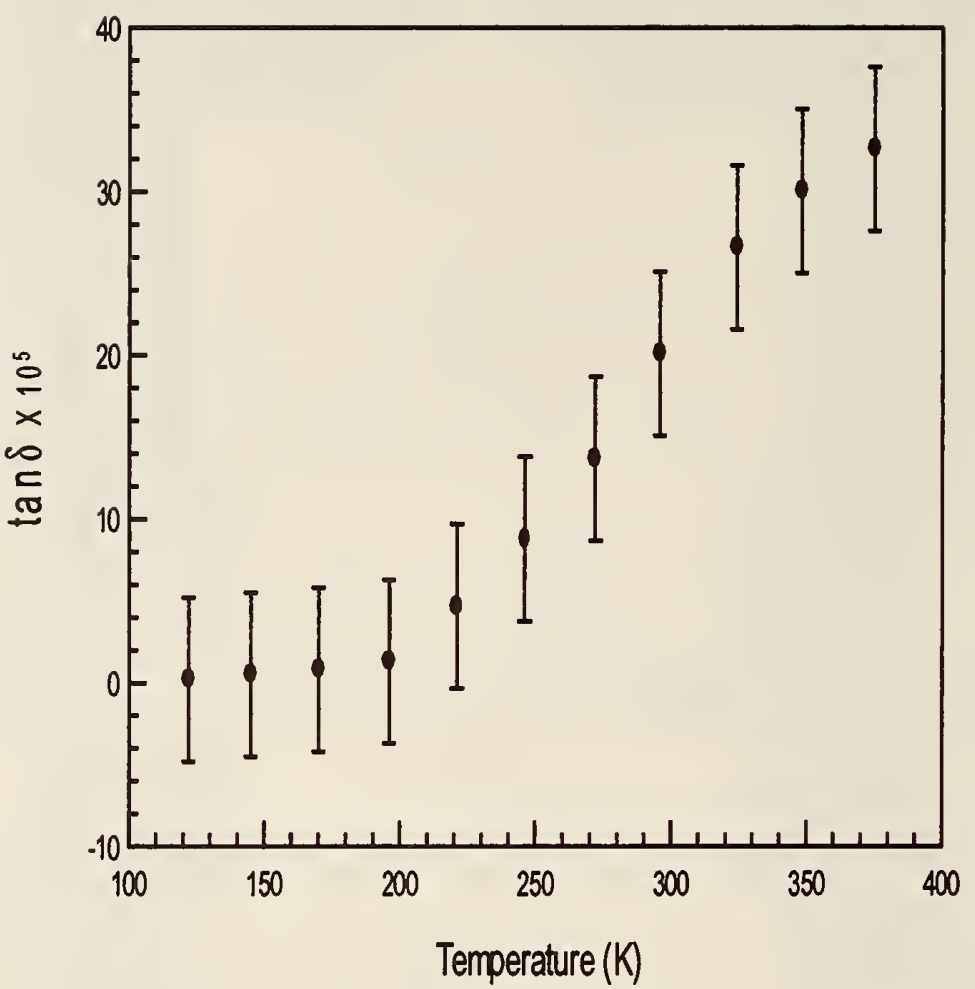

Figure 71. Loss tangent of Teflon as a function of temperature, expanded uncertainies, $k=2$. 


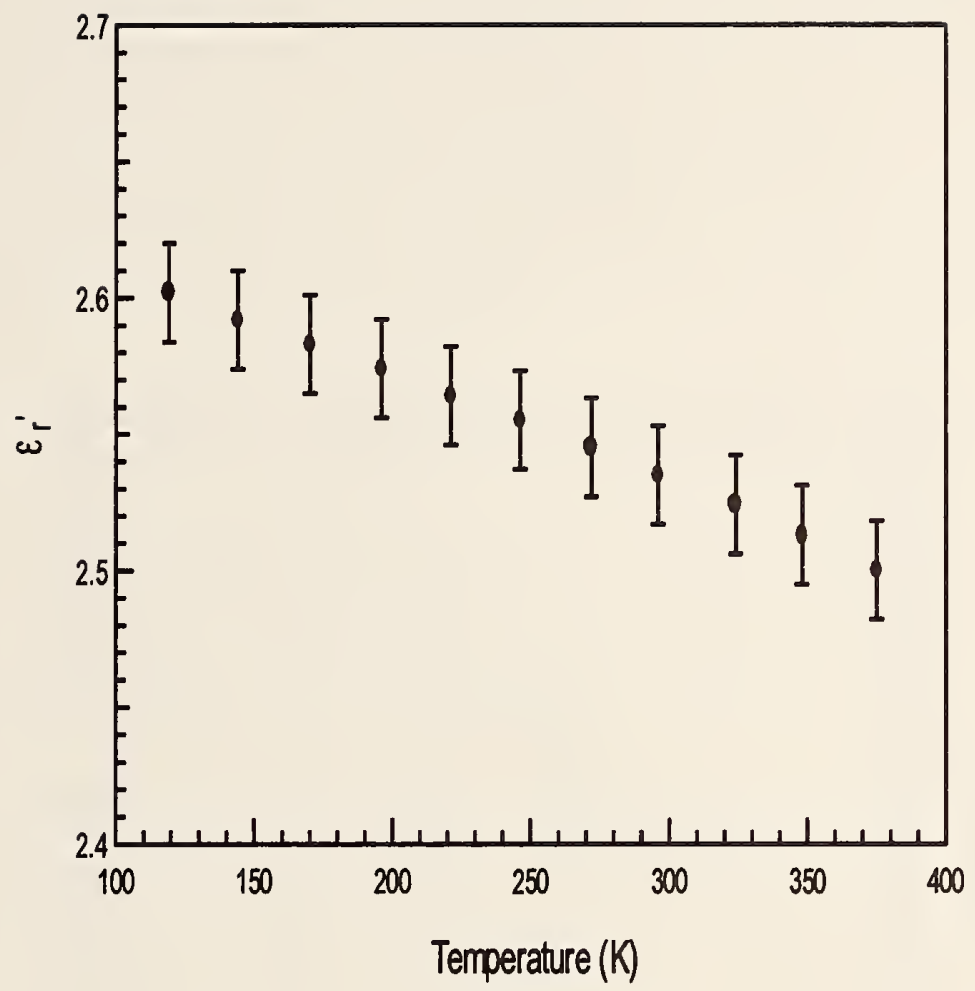

Figure 72. Real part of the permittivity of Rexolite as a function of temperature, expanded uncertainies, $k=2$. 


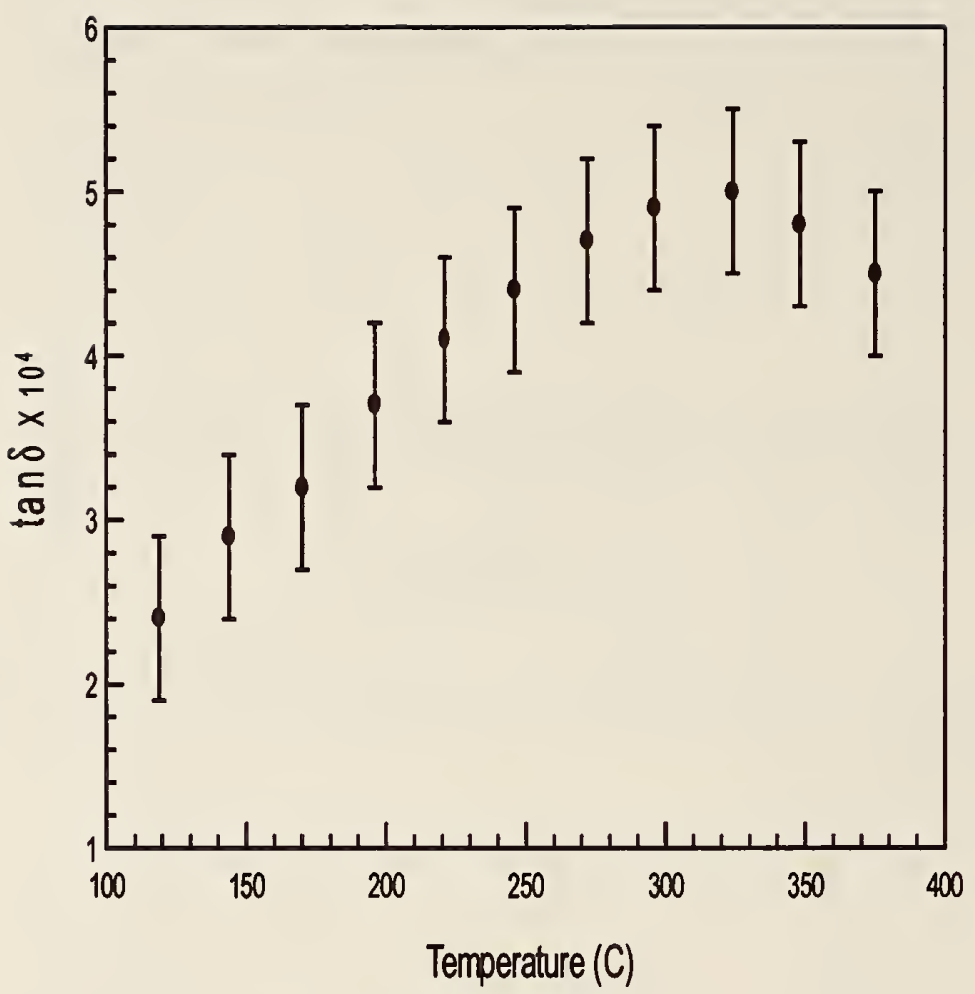

Figure 73. Loss tangent of Rexolite as a function of temperature, expanded uncertainies, $k=2$. 


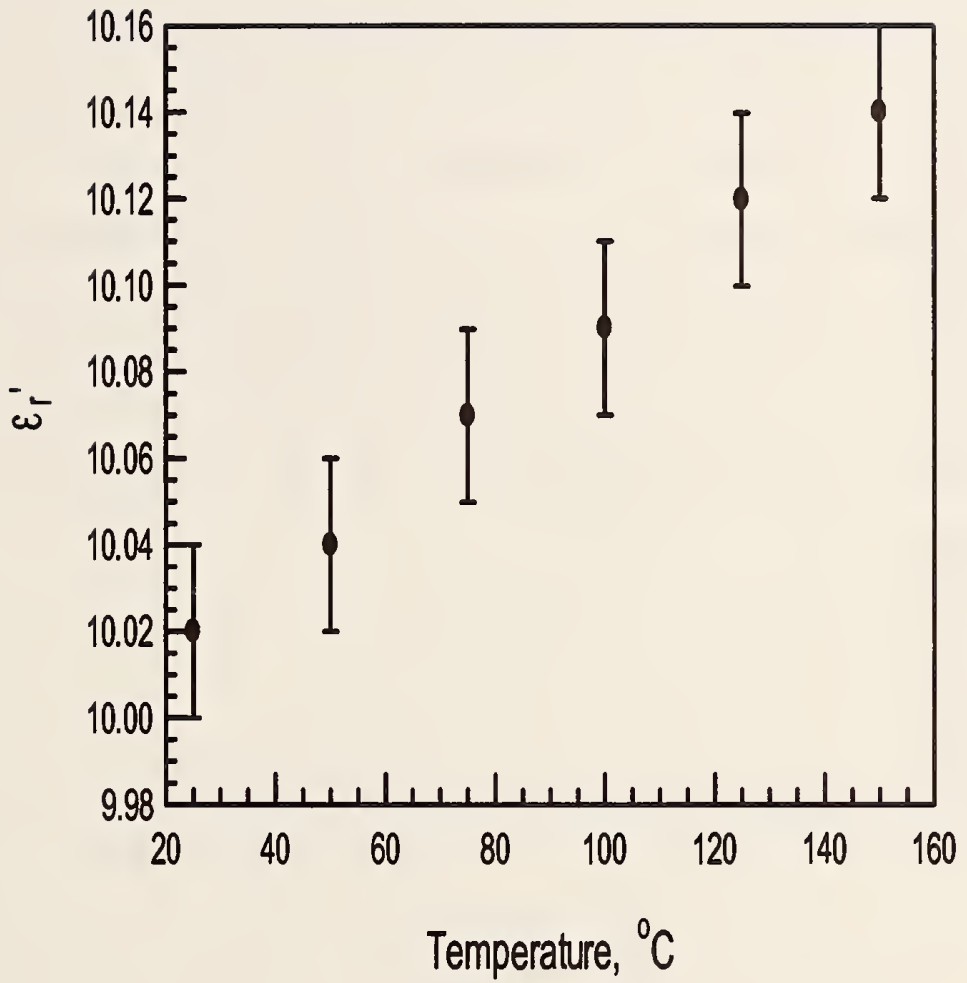

Figure 74. Real part of the permittivity of alumina as a function of temperature, expanded uncertainies, $k=2$. 


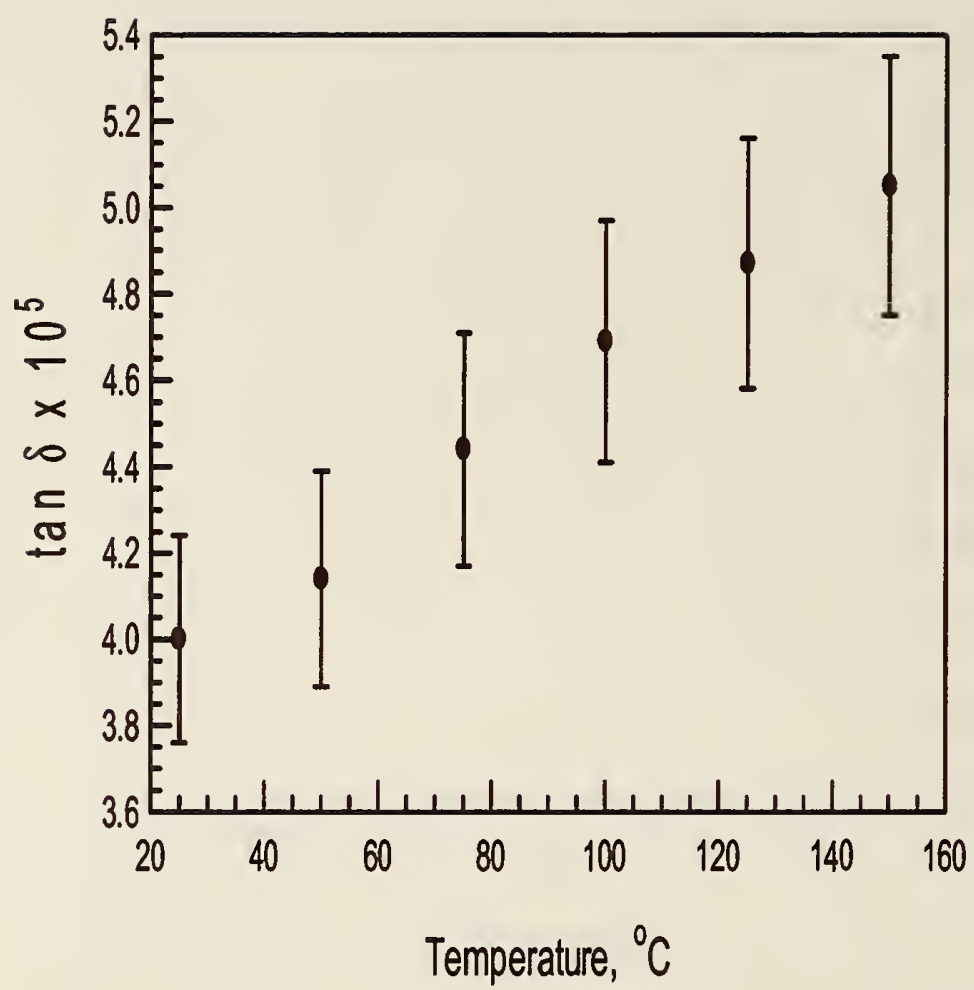

Figure 75. Loss tangent of alumina as a function of temperature, expanded uncertainies, $k=2$. 


\section{Conclusion}

Dielectric measurements for LTCC and PWB materials are required from low megahertz to high gigahertz frequencies. Each technique we have reviewed has a niche in the frequency band, field behavior, and loss characteristics of LTCC, PWB, and substrate materials. One technique alone is insufficient to characterize materials over all of the necessary parameters. Measurements of substrate materials present a metrological challenge because of uncertainty in thickness and anisotropy. Typical PWB materials have a thickness of between $0.2 \mathrm{~mm}$ to $3 \mathrm{~mm}$. Large uncertainties in $\epsilon_{r}^{\prime}$ of thin materials are caused by large uncertainties in thickness. It is critical to use accurate thermal expansion data for temperature dependent permittivity measurements. For methods using conductors, metal losses need to be accounted for and removed. As frequencies increase metal losses and insertion losses increase. 
We would like to acknowledge J. Krupka of Warsaw Technical University and Y. Kantor of Rafael, Israel for measurement assistance; P. Kabos, and R. Geyer, and R. Kaiser for help with measurements and drawings; also G. R. Traut from Rogers Corporation for obtaining samples. We would also like to thank S. Freiman and the NIST Ceramic Division for working with us on collaborations with the LTCC community.

\section{References}

[1] Bahl, I.; Ely, K. Modern microwave substrate materials. Microwave J. 33: 131-146; 1990.

[2] Chang, C. S.; Agrawal, A. P. Fine line thin dielectric circuit board characterization. Proc 44th Electronic Components and Technology Conf.; pp. 564-569. IEEE Comp. Hybrids Mfg. Technol.; 1994.

[3] Chang, C. S. Resistive signal line wiring net designs in multichip modules. IEEE Trans. Comp. Hybrids Mfg. Technol. 16: 909-918; 1993 December.

[4] Westphal, W. P. Techniques of measuring the permittivity and permeability of liquids and solids in the frequency range $3 \mathrm{c} / \mathrm{s}$ to $50 \mathrm{kMc} / \mathrm{s}$. MIT XXXVI; 1950.

[5] Baker-Jarvis, J.; Janezic, M. D.; Grosvenor, J. H.; Geyer, R. G. Transmission/reflection and short-circuit line methods for measuring permittivity and permeability. Natl. Inst. Stand. Technol. NIST Tech. Note 1355; 1994.

[6] Hippel, A. V. Dielectric Materials and Applications. Cambridge, MA: M.I.T. Press; 1954. 
[7] Nicolson, A. M.; Ross, G. F. Measurement of the intrinsic properties of materials by time domain techniques. IEEE Trans. Instrum. Meas. IM-19: 377-382; 1968 November.

[8] Bussey, H. E.; Gray, J. E. Measurement and standardization of dielectric samples. IRE Trans. Instrum. I-11(3): 162-165; 1962.

[9] Baker-Jarvis, J.; Janezic, M. D. Open-ended coaxial probes for nondestructive testing of substrates and circuit boards. Proc. Microwave Processing of Materials IV; Vol. 347; pp. 215-220. MRS; 1994.

[10] Paulter, N. G. Long-term repeatability of a TDR-based printed wiring board dielectric constant measurement system. IEEE Trans. Instrum. Meas. 47: 1469-1473; 1998 December.

[11] Evans, R. Effects of losses on signals in PWB's. IEEE Trans. Comp. Pkg. Mfg. Technol.: Part B 17: 217-222; 1994 May.

[12] Gershon, D.; Calame, J. P.; Birnbolm, A. Complex permittivity measurements abd mixing laws of alumina composites. J. Appl. Phys. 89: 8110-8116; 2001.

[13] Nakayama, A.; Inagaki, M.; Miyauchi, M.; Itoh, N. Dielectric dispersion of polycrystalline aluminum nitride at microwave frequencies. J. Am. Ceram. Soc. 79: 1453-1456; 1996.

[14] Penn, S. J.; McNalford, N.; Templeton, A.; Wang, X.; Xu, M.; Reece, M.; Schrapel, K. Effect of porosity and grain size on the microwave dielectric properties of sintered alumina. J. Am. Ceram. Soc. 80: 1885-1888; 1997.

[15] Cotes, R. Roadmap for interconnection substrates: Ceramic. NEMI Roadmap; 2000. 
[16] Takahashi, A.; Nagai, A.; Mukoh, A.; Wajima, M.; Tsukanishi, K. Low dielectric material for multilayer printed wiring boards. IEEE Trans. Comp. Hybrids Mfg. Technol. 13: 1115-1120; 1990 December.

[17] Jean, J.Ho; Gupta, T. K. Design of low dielectric glass+ceramics for multilayer ceramic substrate. IEEE Trans. Comp. Pkg. Mfg. Technol.:Part B 17: 228-233; 1994 May.

[18] Master, R. N.; Herron, L. W.; Tummala, R. R. Cofiring process for glass/copper multilayer ceramic substrate. Electronic Components and Technology Conference pp. 5-9; 1991.

[19] Brown, R. L. LTCC provides an integrated approach to multichip module design. Electronic Packaging Production 39: 38-40; 1993 October.

[20] Hrovat, M.; Belavic, D. Electronic Packaging for High reliability: Low cost electronics; pp. 161-164. Kluwer Academic Press; London; 1999.

[21] Asai, S.; Funaki, M.; Sawa, H.; Kato, K. Fabrication of an insulated metal substrate (IMS), having an insulating layer with a high dielectric constant. IEEE Trans. Comp. Hybrids Mfg. Technol. 16: 499-504; 1993 August.

[22] Traut, G. R. Clad laminates of PTFE composites for microwave antennas. Microwave J. 23: 47-53; 1980 November.

[23] Sasaki, A.; Shimada, Y. Electrical design technology for low dielectric constant multilayer ceramic substrate. IEEE Trans. Comp. Hybrids Mfg. Technol. 15: 56-62; 1992 February.

[24] Deutsch, A.; Surovic, C.; Lanzetta, A. Broadband characterization of low dielectric constant and low dielectric loss cytuf cyanate ester printed circuit 
board material. IEEE Trans. Comp. Pack. Mfg. Part B 19: 331-336; 1996 May.

[25] Ibarra, A.; Gonzalez, M.; Vila, R. Dielectric properties of some organic RFsubstrates. Micro. Eng. Europe 1: 25-34; 1998 May.

[26] Laverghetta, T. S. Microwave Materials and Fabrication Techniques. Boston: Artech House; 2000.

[27] Baker-Jarvis, J. Transmission/reflection and short-circuit line permittivity measurements. Natl. Inst. Stand. Technol.; 1990 July.

[28] Bussey, H. E.; Steinert, L. A. Exact solution for a gyromagnetic sample and measurements on a ferrite. IRE Trans. Microwave Theory Tech. MTT-6: 72-76; 1958 January.

[29] ASTM-D3380-90 standard test method for relative permittivity and dissipation factor for polymer-based microwave circuit substrates. 1990 Annual Book of American Society for Testing of Materials (ASTM) 10.01; 1990.

[30] Papoulis, A. The Fourier Integral and Its Applications. New York: McGrawHill; 1987.

[31] Holloway, C. L.; Kuester, E. F. Power loss associated with conducting and rough surfaces. IEEE Trans. Microwave Theory Tech. 48: 1601-1610; 2000 October.

[32] Janezic, M. D.; Williams, D. F. Permittivity characterization from transmission-line measurements. Vol. 3; pp. 1343-1346. IEEE MTT-S Intl. Symp.; 1997 December. 
[33] Osaki, S. Microwave frequency dielectric properties of poly (vinylidene fluoride) films. J. Poly. Sci. 10.01; 1995.

[34] McPhun, M.; Mehmet, K. Thin film dielectric measurements. Guilford, U.K.: IPC Science and Technology; 1973.

[35] Taylor, B. N.; Kuyatt, C. E. Guidelines for evaluating and expressing the uncertainty of NIST measurement results. Natl. Inst. Stand. Technol. NIST Tech. Note 1297; 1994 September.

[36] Harrington, R. D.; Powell, R. C.; Haas, P. H. A re-entrant cavity for measurement of complex permeability in the very-high frequency region. J. Res. Nat. Bur. Stand. (U.S.) 56(3): 129-133; 1956.

[37] Works, C. N.; Dakin, T. W.; Boggs, F. W. A resonant cavity method for measuring dielectric properties at ultra-high frequencies. AIEE Trans. 63: 1092$1098 ; 1944$.

[38] Baker-Jarvis, J.; Riddle, B. F. Dielectric measurements using a reentrant cavity. NIST Tech. Note 1384; 1996 November.

[39] Kaczkowski, A.; Milewski, A. High-accuracy wide-range measurement method for determination of complex permittivity in re-entrant cavity: Part A, theoretical analysis of the method. IEEE Trans. Microwave Theory Tech. MTT-28(3): $225-228 ; 1980$.

[40] Kaczkowski, A.; Milewski, A. High-accuracy wide-range measurement method for determination of complex permittivity in reentrant cavity, Part B- Experimental analysis of measurement errors. IEEE Trans. Microwave Theory Tech. MTT-28(3): 228-231; 1980. 
[41] Kent, G. Nondestructive measurements of substrate permittivity. Proc. Third Intl. Symp. on Recent Advances in Microwave Technology; pp. 1-4; 1991.

[42] Kent, G. An evanescent-mode tester for ceramic dielectric substrates. IEEE Trans. Microwave Theory Tech. MTT-36: 1451-1454; 1988 October.

[43] Kent, G. A dielectrometer for the measurement of substrate permittivity. Microwave J. pp. 72-82; 1991 December.

[44] Janezic, M. D.; Jargon, J. A. Complex permittivity determination from propagation constant measurement. IEEE Microwave Guided Wave Lett. 9: 76-78; 1999 February.

[45] Vanzura, E. J.; Geyer, R. G.; Janezic, M. D. The NIST 60-millimeter diameter cylindrical cavity resonator: Performance evaluation for permittivity measurements. Natl. Inst. Stand. Technol. Tech. Note 1354; 1993 August.

[46] Damaskos, N. Measuring dielectric constants of low loss materials using a broadband cavity technique. Microwave J. 38: 140-147; 1995 September.

[47] Kobayashi, Y.; Katoh, M. Microwave measurement of dielectric properties of low-loss materials by the dielectric rod resonator method. IEEE Trans. Microwave Theory Tech. MTT-33: 586-592; 1985.

[48] Nishikawa, T.; Tanaka, H.; Ishikawa, Y. Noncontact relative measurement method for complex permittivity of ceramic substrate. Digest IECE of Japan Symp. pp. 154-155; 1986.

[49] Maj, S.; Pospieszalski, M. A composite multilayered cylindrical dielectric resonator. IEEE MTT-S Digest; pp. 190-192; 1984 May. 
[50] Delaballe, J. Complex permittivity measurement of MIC substrates. Electron. Telecommun. 35: 80-83; 1981.

[51] Courtney, W. E. Analysis and evaluation of a method of measuring the complex permittivity and permeability of microwave insulators. IEEE Trans. Microwave Theory Tech. MTT-18: 467-485; 1970 August.

[52] Kajfez, D.; Guillon, P. Dielectric Resonators. New York: Arch House; 1986.

[53] Klein, N. Properties and applications of HTS-shielded dielectric resonators: A state-of the-art report. IEEE Trans. Microwave Theory Tech. 44: 1369-1373; 1996.

[54] Geyer, R.; Vanderah, T.; Jones, C.; Krupka, J. Complex permeability measurements of ferrite ceramics used in wireless communications. J. Am. Ceram. Soc. 88: 93-111; 1998.

[55] Geyer, R.; Baker-Jarvis, J.; Vanderah, T.; Mantese, J. Complex permittivity and permeability estimation of composite electroceramics. J. Am. Ceram. Soc. 88: $115-120 ; 1998$.

[56] Krupka, J.; Geyer, R. G. Complex permeability of demagnetized microwave ferrites near and above gyromagnetic resonance. IEEE Trans. Magn. 32: 1924$1933 ; 1996$.

[57] Krupka, J.; Derzakowski, F.; Modelski, J. Method of measuring the complex permittivity and permeability of ferrite substrate plates by means of two section dielectric resonator with TE01 $\delta$ mode. Digest MIKON; pp. 393-397; 1991 September. 
[58] Krupka, J.; Klinger, M.; Kuhn, M.; Baranyak, A.; Stiller, M.; Hinken, J. Surface resistance measurements of HTS films by means of sapphire dielectric resonators. IEEE Trans. Appl. Supercond. 3: 3043-3048; 1993.

[59] Shen, Z.-Y.; Wilker, C.; Pang, P.; Holstein, W.; Face, D.; Kountz, D. High T sapphire microwave resonator with extremely high Q-values up to $90 \mathrm{~K}$. IEEE Trans. Microwave Theory Tech. 40: 2424-2428; 1992 December.

[60] Krupka, J.; Blondy, P.; Cros, D.; Guillon, P.; Geyer, R. G. Whispering-gallery modes and permeability tensor measurements in magnetized ferrite resonators. IEEE Trans. Microwave Theory Tech. 44: 1097-1102; 1996.

[61] Krupka, J. Measurement of all permeability tensor components and effective linewidth of microwave ferrites using dielectric ring resonators. IEEE Trans. Microwave Theory Tech. 39: 1148-1157; 1991.

[62] Luiten, A. N.; Tobar, M.; Krupka, J.; Woode, R.; Ivanov, E. N.; Mann, A. G. Microwave properties of a rutile resonator between 2 and $10 \mathrm{~K}$. J. Appl. Phys. 31: 1383-1391; 1998 June.

[63] Tobar, M.; Krupka, J.; Woode, R. A. Anisotropic complex permittivity measurements of mono-crystalline between 10 and $300{ }^{\circ} \mathrm{K}$. J. Appl. Phys. 31: 1383-1391; 1998 June.

[64] Rayleigh, L. The problem of the whispering gallery. Phil. Mag. 20: 1001-1004; 1910.

[65] Wait, J. R. Electromagnetic whispering-gallery modes in a dielectric rod. Radio Sci. 2: 1005-1017; 1967.

[66] Vedrenne, C.; Arnaud, J. Whispering-gallery modes in dielectric resonators. IEE Proc. H. Microwave, Antennas Propagation 129: 183-187; 1982. 
[67] Weiming, O.; Gardner, C. G.; Long, S. A. Nondestructive measurement of a dielectric layer using surface electromagnetic waves. IEEE Trans. Microwave Theory Tech. MTT-31(3): 255-260; 1983 March.

[68] Alexander, R. W.; Jr.; Bell, R. J. The use of surface electromagnetic waves to measure material properties. J. Noncryst. Solids 19: 93-103; 1975.

[69] Krupka, J.; Cros, D.; Aubourg, M.; Guillon, P. Study of whispering gallery modes in anisotropic single-crystal dielectric resonators. IEEE Trans. Microwave Theory Tech. 42(1): 56-61; 1994.

[70] Lynch, A. C. Precise measurement of complex permittivity and permeability in the millimeter region by a frequency domain technique. IEEE Trans. Instrum. Meas. 23: 425-430; 1974.

[71] Afsar, M. A. Dielectric measurements of millimeter-wave materials. IEEE Trans. Microwave Theory Tech. MTT-32(12): 1598-1609; 1984.

[72] Kogelnik, H.; Li, T. Laser beams and resonators. Proc., IEEE 54: 1312-1328; 1966.

[73] Jones, R. G. Precise dielectric measurements at $35 \mathrm{GHz}$ using an open microwave resonator. Proc. IEE 123: 285-290; 1976.

[74] Clarke, R. N.; Rosenberg, C. B. Fabry-Perot and open resonators at microwave and millimeter wave frequencies, 2-300 GHz. J. Phys. E.: Sci. Instrum. 15(9): $9-24 ; 1982$.

[75] Hirvonen, T. M.; Vainikainen, P.; Lozowski, A.; Raisanen, A. V. Measurement of dielectrics at $100 \mathrm{GHz}$ with an open resonator connected to a network analyzer. IEEE Trans. Instrum. Meas. 45: 780-786; 1996. 
[76] Baker-Jarvis, J.; Vanzura, E.; Kissick, W. Improved technique for determining complex permittivity with the transmission/reflection method. IEEE Trans. Microwave Theory Tech. 38(8): 1096-1103; 1990 August.

[77] Baker-Jarvis, J.; Geyer, R. G.; Domich, P. D. A nonlinear optimization technique for permittivity and permeability determination. IEEE Trans. Instrum. Meas. 41(5): 646-652; 1992.

[78] Vanzura, E. J.; Baker-Jarvis, J. R.; Grosvenor, J. H.; Janezic, M. D. Intercomparison of permittivity measurements using the transmission/reflection method in 7-mm coaxial transmission lines. IEEE Trans. Microwave Theory Tech. MTT-42(11): 2063-2070; 1994 November.

[79] Baker-Jarvis, J. Linear and nonlinear electromagnetic response. Digest URSI. URSI; 1993 January. University of Colorado, Boulder, CO.

[80] Gardiol, F. E.; Sphicopoulos, T.; Teodoridis, V. The reflection of open-ended circular waveguides: Application to nondestructive measurement of materials. Button, K. J., editor, Rev. Infrared Millimeter Waves; pp. 325-364; New York; 1983. Plenum.

[81] Teodoridis, V.; Sphicopoulos, T.; Gardiol, F. E. The reflection from an openended rectangular waveguide terminated by a layered dielectric medium. IEEE Trans. Microwave Theory Tech. MTT-33(5): 359-366; 1985 May.

[82] Bringhurst, S.; Iskander, M. F. New metalized ceramic coaxial probe for hightemperature broadband dielectric properties of low permittivity materials. Microwaves: Theory and Application in Materials Processing II; pp. 503-510. Amer. Ceram. Soc.: Ceramics Trans.; 1993. 
[83] Li, L. L.; Ismail, N. H.; Taylor, L. S.; Davis, C. C. Flanged coaxial microwave probes for measuring thin moisture layers. IEEE Trans. Biomed. Eng. 39(1): 49-57; 1992 January.

[84] Langhe, P. D.; Blomme, K.; Martens, L.; Zutter, D. D. Measurement of lowpermittivity materials based on a spectral domain analysis for the open-ended coaxial probe. IEEE Trans. Instrum. Meas. IM-42(9): 879-886; 1993 October.

[85] Levine, H.; Papas, C. H. Theory of the circular diffraction antenna. J. Appl. Phys. 22(1): 29-43; 1951 January.

[86] Baker-Jarvis, J.; Janezic, M. D.; Domich, P. D.; Geyer, R. G. Analysis of an open-ended coaxial probe with lift-off for nondestructive testing. IEEE Trans. Instrum. Meas. 43(5): 711-718; 1994 October.

[87] Hodgetts, T. E. The open-ended coaxial line: A rigorous variational treatment. Royal Signals and Radar Establishment 4331; 1989.

[88] Jenkins, S.; Hodgetts, T. E.; Symm, G. T.; Warhamm, A. G. P.; Clarke, R. N. Comparison of three numerical treatments for the open-ended coaxial line sensor. Electron. Lett. 26: 234-235; 1990.

[89] Ganchev, S.; Bakhtiari, S.; Zoughi, R. A novel numerical technique for dielectric measurements of lossy dielectrics. IEEE Trans. Instrum. Meas. 41: 361-365; 1992 June.

[90] Baker-Jarvis, J.; Janezic, M. D. Analysis of a two-port flanged coaxial holder for shielding effectiveness and dielectric measurements of thin films and thin materials. IEEE Trans. Electromag. Compat. pp. 67-70; 1996 February. 
[91] Stuchly, M. A.; Stuchly, S. S. Coaxial line reflection methods for measuring dielectric properties of biological substances at radio and microwave frequenciesA review. IEEE Trans. Instrum. Meas. IM-29: 176-183; 1980.

[92] Marcuvitz, N. Waveguide Handbook. New York: Dover Publications; 1951.

[93] Nozaki, R.; Bose, T. Complex permittivity measurements by time-domain spectroscopy. IEEE Trans. Instrum. Meas. 39: 945-951; 1990.

[94] Belhadj-Tahar, N.-E.; Fourier-Lamer, A.; Chanterac, H.de. Broadband simultaneous measurement of complex permittivity and permeability using a coaxial discontinuity. IEEE Trans. Microwave Theory Tech. 38(1): 1-7; 1990 January.

[95] Bussey, H. E. Measurement of rf properties of materials: A survey. Proc., IEEE 55(6): 1046-1053; 1967 June.

[96] Hartshorn, L.; Ward, W. H. The measurement of the permittivity and power factor of dielectrics at frequencies $10^{4}$ to $10^{8}$ cycles per second. J. Inst. Elec. Eng. 79: 597-609; 1936.

[97] Broadhurst, M. G.; Bur, A. J. Two-terminal dielectric measurements up to $6 \times 10^{8}$ Hz. J. Res. Nat. Bur. Stand. (U.S.) 69C(3): 165-172; 1965.

[98] Jenkins, S.; Hodgetts, T. E.; Clarke, R. N.; Preece, A. W. Dielectric measurements on reference liquids using automatic network analyzers and calculable geometries. Meas. Sci. Tech. 1: 691-702; 1990.

[99] New technologies for wide impedance range measurements to $1.8 \mathrm{GHz}$. Hewlett Packard; 1994.

[100] ASTM-D1531: Relative permittivity and dissipation factor by fluid displacement procedures. 1990 Annual Book of ASTM Standards 10.02; 1990. 
[101] Marks, R. B.; Williams, D. F. Characteristic impedance determination using propagation constant measurement. IEEE Microwave Guided Wave Lett. 1: 141-143; 1991 June.

[102] Marks, R. B. A multiline calibration for network analyzers. IEEE Trans. Microwave Theory and Techniques 39: 1205-1215; 1991.

[103] Ramo, S.; Whinnery, J. R.; Duzer, T. V. Fields and Waves in Communication Electronics. New York: John Wiley and Sons; 1984.

[104] Marks, R. B.; Williams, D. F. A general waveguide circuit theory. J. Res. Natl. Inst. Stds. Technol. 97: 533-562; 1992 October.

[105] Beatty, R. W. Insertion loss concepts. Proc. IEEE pp. 663-671; 1964.

[106] Ginzton, E. L. Microwave Measurements. New York: McGraw-Hill Book Company; 1957.

[107] Pozar, D. M. Microwave Engineering. New York: Addison-Wesley Publishing Company; 1993.

[108] Paulter, N. G. A fast and accurate way for measuring the dielectric constant of printed wiring board materials. IEEE Trans. Components Pack. Mfg. Technol. 19C: 214-225; 1996 July.

[109] Lewis, R. L. Relative permittivity measurement of square copper-laminated substrates using the full-sheet resonance technique. Natl. Inst. Stand. Technol. NISTIR 5053; 1997.

[110] Woolaver, G. I. Accurately measure dielectric constant of soft substrates. Microwave \& RF 24: 153-158; 1990 August. 
[111] Collin, R. E. Field Theory of Guided Waves. New York: IEEE Press; 1991.

[112] Weir, W. B. Automatic measurement of complex dielectric constant and permeability at microwave frequencies. Proc. IEEE 62(1): 33-36; 1974 January.

[113] Goldfarb, R. B.; Bussey, H. E. Method for measuring complex permeability at radio frequencies. Rev. Sci. Instrum. 58(4): 624-627; 1987.

[114] Mattar, K. E.; Watters, D. G.; Brodwin, M. E. Influence of wall contacts on measured complex permittivity spectra at coaxial line frequencies. IEEE Trans. Microwave Theory Tech. MTT-39(3): 532; 1991.

[115] York, R. A.; Compton, R. C. An automated method for dielectric constant measurements of microwave substrates. Microwave J. 33: 115-121; 1990 March.

[116] Waldron, R. A. Theory of a strip-line cavity for measurement of dielectric constants and gyromagnetic resonance line-widths. IEEE Trans. Microwave Theory Tech. IM-12: 123-131; 1964 January.

[117] Wheeler, H. A. Transmission-line properties of parallel-wide strips separated by a dielectric sheet. IEEE Trans. Microwave Theory Tech. MTT-13: 172-185; 1965 March.

[118] Getsinger, W. J. Microstrip dispersion model. IEEE Trans. Microwave Theory Tech. IM-21: 34-39; 1973 January.

[119] Traut, G. R. Electrical test methods for microwave PCB's. Microwave J. 24: 73-79; 1981 August.

[120] Howell, J. Q. A quick, accurate method to measure the dielectric constant of microwave integrated circuits. IEEE Trans. Microwave Theory Tech. MTT-21: 142-143; 1973 March. 
[121] Napoli, L. S. A simple technique for the accurate determination of microwave dielectric constant for microwave integrated circuits. IEEE Trans. Microwave Theory Tech. MTT-19: 664-667; 1971 July.

[122] Gupta, K. C.; Garg, R.; Bahl, I. J. Microwave Lines and Slotlines. Norwood, MA: Artech House; 1979.

[123] Schneider, M. V. Microstrip lines for microwave integrated circuits. Bell Syst. Tech. J. pp. 1421-1444; 1968 May-June.

[124] Fidanboylu, K. M.; Riad, S. M.; Elshabini-Riad, A. A new time-domain approach for determining the complex permittivity using stripline geometry. IEEE Trans. Instrum. Meas. IM-39(6): 940-944; 1990 December.

[125] Bahl, I. J.; Stuchly, S. S. Analysis of a microstrip covered with a lossy dielectric. IEEE Trans. Microwave Theory Tech. MTT-28(2): 104-109; 1980 February.

[126] Tanaka, H.; Okada, F. Precise measurements of dissipation factor in microwave printed circuit boards. IEEE Trans. Instrum. Meas. IM-38(2): 509-514; 1989 April.

[127] Olyphant, M.; Ball, J. H. Stripline methods for dielectric measurements at microwave frequencies. IEEE Trans. Electron. Insul. EI-5(1): 26-32; 1970 March.

[128] Das, N. K.; Voda, S. M.; Pozar, D. M. Two methods for the measurement of substrate dielectric constant. IEEE Trans. Microwave Theory Tech. MTT-35: 636-641; 1987 July.

[129] Clapeau, M.; Guillon, P.; Garault, Y. Resonant frequencies of superimposed dielectric resonators: Application to determination of the local dielectric per- 
mittivity of MIC substrates. Proc., 7th European Microwave Conference; pp. $545-549 ; 1977$.

[130] Laursen, K.; Hertling, D.; Berry, N.; Bidstrup, S. A.; Kohl, P.; Arroz, G. Measurement of the electrical properties of high performance dielectric materials for multichip modules. Proc., IEPS; pp. 11-13; 1993. San Diego, CA.

[131] Pannell, R. M.; Jervis, B. W. Two simple methods for measurement of the dielectric permittivity of low-loss microstrip substrates. Microwave Theory Tech. 29: 383-386; 1981 April.

[132] Pucel, R. A.; Masse, D. J.; Hartwig, C. P. Losses in microstrip. IEEE Trans. Microwave Theory Tech. MTT-16: 342-350; 1968 June.

[133] Holloway, C. L.; Kuester, E. F. Edge shape effects and quasi-closed form expressions for the conductor loss of microstrip lines. Radio Sci. 29: 539-559; 1994 May-June.

[134] Holloway, C. L.; Kuester, E. F. A quasi-closed form expression for the conductor loss of CPW lines, with an investigation of edge shape effects. IEEE Trans. Microwave Theory Tech. 12: 2695-2701; 1995 December.

[135] Booth, J. C.; Holloway, C. L. Conductor loss in superconducting planar structures: Calculations and measurements. IEEE Trans. Microwave Theory Tech. 47: 769-773; 1999 June.

[136] Holloway, C. L. Expressions for the conductor loss of strip-line and coplanarstrip (cps) structures. Microwave Opt. Technol. Lett. 25: 162-168; 2000 May.

[137] Kuester, E. Accurate approximations for a function appearing in the analysis of microstrip. IEEE Trans. Microwave Theory Tech. 32: 131-133; 1984. 
[138] Holloway, C. L.; Hufford, G. A. Internal inductance and conductor loss associated with the ground plane of a microstrip line. IEEE Trans. Electromagn. Compat. 39: 73-78; 1997 May.

[139] Leontovich, M. A. Approximate boundary conditions for electromagnetic fields at the surface of a highly conducting body. Issledovaniya po Rasprostraneniyu Radiovoln, part 2 5-12: 351-355; 1985.

[140] Fjelstad, J. Printed circuit technology materials and processes. The Institute for Interconnecting and Packaging Electronic Circuits 1; 1995.

[141] Laverghetta, T. S. Microwave Materials and Fabrication Techniques. New York: Artech House; 1990.

[142] IPC-TM-650 test methods manual. The Institute for Interconnecting and Packaging Electronic Circuits 2.5.5.5; 1997.

[143] Holloway, C.; Kuester, E. Power loss associated with conducting and superconducting rough interfaces. IEEE Trans. Microwave Theory Tech. 48: 1601-1610; 2000.

[144] Amey, D. L.; Cutilla, J. P. Microwave properties of ceramic materials. pp. 267-272; 1991.

[145] Bernard, P. A.; Gautray, J. M. Measurement of dielectric constant using a microstrip ring resonator. IEEE Trans. Microwave Theory Tech. 39: 592-595; 1991.

[146] Vasudevan, S.; Shaikh, A. Microwave characterization of low temperature cofired ceramic system. pp. 152-161. Intl. Symp. on Advanced Packaging Materials; 1997. 
[147] Sarabandi, K.; Li., E. S. Microstrip ring resonators for soil moisture measurements. IEEE Trans. Geosci. Remote Sensing 35: 1223-1231; 1997.

[148] Deutsch, A.; Swaminathan, M. Measurement of dielectric anisotropy of bpdapda polyimide in multilayer thin-film packages. IEEE Trans. Comp. Pack. Manuf. Part B 17: 486-492; 1994 November.

[149] Vanzura, E. J.; Weil, C. M.; Williams, D. F. Complex permittivity measurements of gallium arsenide using a high-performance resonant cavity. Digest; pp. 103-104. Conf. on Precision Electromagnetic Measurements; 1992.

[150] Krupka, J.; Baker-Jarvis, J.; Geyer, R. G.; Ceremuga, J. Measurement of the complex permittivity of microwave circuit board substrates using split dielectric resonator and reentrant cavity. Dielectric Materials Measurements and Applications; pp. 21-24. IEE; 1996.

[151] Baker-Jarvis, J.; Geyer, R.; Grosvenor, J.; Janezic, M.; Jones, C.; Riddle, B.; Weil, C.; Krupka, J. Dielectric measurements on low-loss materials: A comparison of techniques. IEEE Trans. Dielect. Insul. 5: 571-577; 1998 August.

[152] Bussey, H. E.; Gray, J. E.; Bamberger, E. C.; Rushton, E.; Russell, G.; Petley, B. W.; Morris, D. International comparison of dielectric measurements. IRE Trans. Instrum. Meas. IM-13(4): 305-311; 1964 December.

[153] Bussey, H. E.; Morris, D.; Zaltsman, E. B. International comparison of complex permittivity at $9 \mathrm{GHz}$. IEEE Trans. Instrum. Meas. IM-23(3): 235-238; 1974 September.

[154] Westphal, W. P.; Sils, A. Dielectric constant and loss data. MIT, Air Force Materials Lab. AFML-TR-72-39; 1972. 
[155] Tummala, R. R. Multichip packaging: A tutorial. Proc., IEEE 80: 1924-1941; 1992.

[156] Westphal, W. B. Dielectric constant and loss data. MIT, Air Force Materials Lab. AFML-TR-74-250; 1980.

[157] Weast, R. C. Handbook of Chemistry and Physics, 56th edition. Cleveland, OH: CRC Press; 1976.

[158] Tummala, R. R. Ceramic and glass-ceramic packaging in the 1990s. J. Am. Ceram. Soc. 74: 895-908; 1991.

[159] Touloukian, Y. S. Thermal Expansion: Non metallic solids. New York, NY: Plenum; 1977.

[160] Jean, J.Ho. Design of low dielectric glass+ceramics for multilayer ceramic substrates. IEEE Trans. Comp. Pkg. Mfg. Technol.: Part B 17: 228-233; 1994.

[161] Yeh, C.; Ume, C.; Fulton, R. E.; Wyatt, K. W.; Stafford, J. W. Correlation of analytical and experimental approaches to determine thermally induced PWB warpage. IEEE Trans. Comp. Hybrids Mfg. Technol. 16: 986-994; 1993 December.

[162] Neelakanta, P. Electromagnetic Materials. London: CRC Press; 1995.

[163] Sihvola, A. Electromagnetic Mixing Formulas and Applications. London: Institution of Electrical Engineers; 1999. 


\section{Appendix A: Mixture Equations}

It is often of interest to estimate the permittivity of a mixture of a number of distinct materials. The effective permittivity of a mixture $\epsilon_{\text {eff }}$ of constituents with permittivity $\epsilon_{i}$ and volume fractions $\theta_{i}$ can be approximated in various ways. For an arbitrary number of constituents the following mixture equation apply [160]

$$
\frac{\epsilon_{e f f}^{\prime}-\epsilon_{1}^{\prime}}{3 \epsilon_{1}^{\prime}}=\frac{\theta\left(\epsilon_{2}^{\prime}-\epsilon_{1}^{\prime}\right)}{\left(\epsilon_{2}^{\prime}+2 \epsilon_{1}^{\prime}\right)}
$$

The Bruggeman equation is useful for binary mixtures [162]

$$
\theta_{1} \frac{\epsilon_{e f f}^{\prime}-\epsilon_{1}^{\prime}}{\epsilon_{1}^{\prime}+2 \epsilon_{e f f}^{\prime}}=\theta_{2} \frac{\epsilon_{2}^{\prime}-\epsilon_{e f f}^{\prime}}{\epsilon_{2}^{\prime}+2 \epsilon_{e f f}^{\prime}}
$$

or the Maxwell-Garnett mixture equation can be used [162]

$$
\frac{\epsilon_{e f f}^{\prime}-\epsilon_{2}^{\prime}}{\epsilon_{e f f}^{\prime}+2 \epsilon_{2}^{\prime}}=\theta_{1} \frac{\epsilon_{1}^{\prime}-\epsilon_{2}^{\prime}}{\epsilon_{1}^{\prime}+2 \epsilon_{2}^{\prime}}
$$

where $\epsilon_{1}^{\prime}$ is $\epsilon_{r}^{\prime}$ of the matrix and $\epsilon_{2}^{\prime}$ is $\epsilon_{r}^{\prime}$ of the filler [163]. 


\section{Appendix B: Broadband-Plastic Measurements}

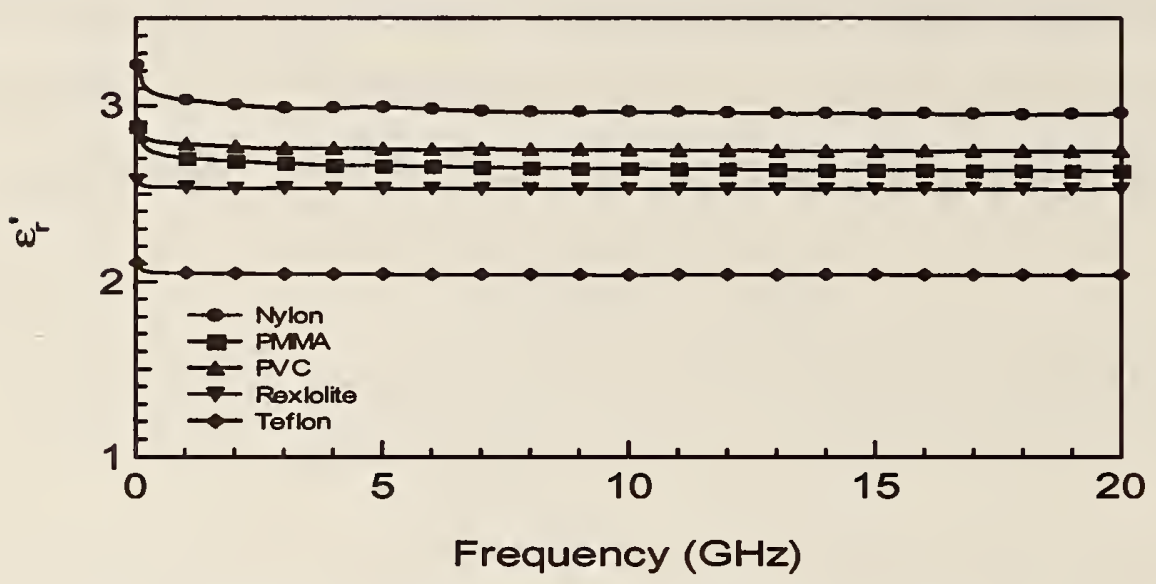

Figure 76. Measurements on $\epsilon_{r}^{\prime}$ on polymers.

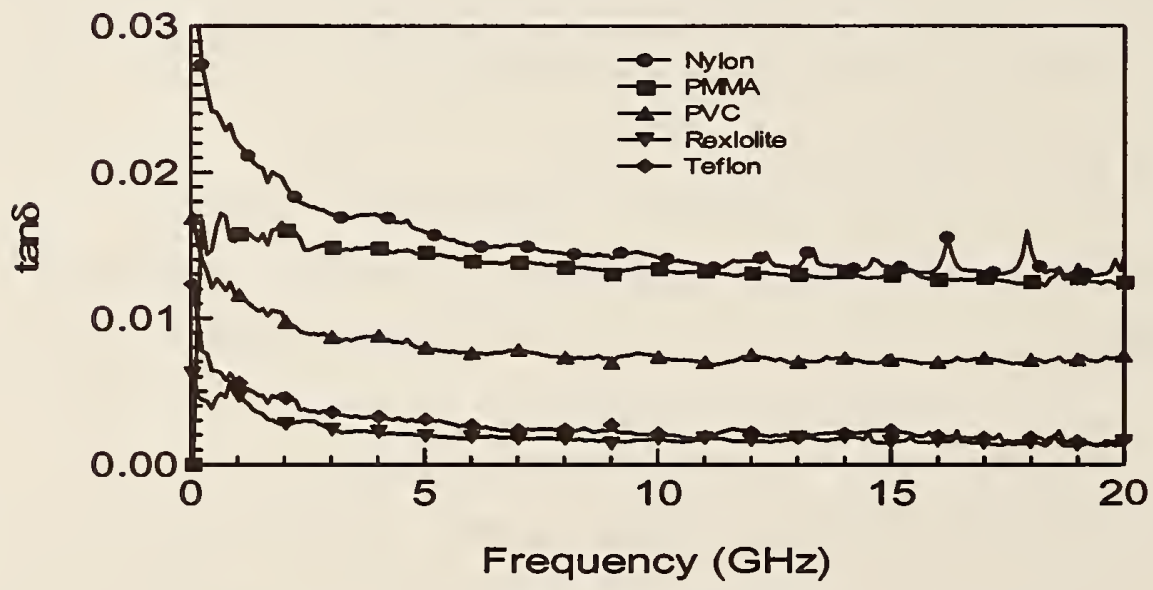

Figure 77. Measurements on $\tan \delta$ on polymers. 


\section{MIST Technical Publications}

\section{Periodical}

Journal of Research of the National Institute of Standards and Technology-Reports NIST research and development in those disciplines of the physical and engineering sciences in which the Institute is active. These include physics, chemistry, engineering, mathematics, and computer sciences. Papers cover a broad range of subjects, with major emphasis on measurement methodology and the basic technology underlying standardization. Also included from time to time are survey articles on topics closely related to the Institute's technical and scientific programs. Issued six times a year.

\section{Nonperiodicals}

Monographs-Major contributions to the technical literature on various subjects related to the Institute's scientific and technical activities.

Handbooks-Recommended codes of engineering and industrial practice (including safety codes) developed in cooperation with interested industries, professional organizations, and regulatory bodies.

Special Publications-Include proceedings of conferences sponsored by NIST, NIST annual reports, and other special publications appropriate to this grouping such as wall charts, pocket cards, and bibliographies.

Applied Mathematics Series-Mathematical tables, manuals, and studies of special interest to physicists, engineers, chemists, biologists, mathematicians, computer programmers, and others engaged in scientific and technical work.

National Standard Reference Data Series-Provides quantitative data on the physical and chemical properties of materials, compiled from the world's literature and critically evaluated. Developed under a world wide program coordinated by NIST under the authority of the National Standard Data Act (Public Law 90-396). NOTE: The Journal of Physical and Chemical Reference Data (JPCRD) is published bi-monthly for NIST by the American Chemical Society (ACS) and the American Institute of Physics (AIP). Subscriptions, reprints, and supplements are available from ACS, 1155 Sixteenth St., NW, Washington, DC 20056.

Building Science Series-Disseminates technical information developed at the Institute on building materials, components, systems, and whole structures. The series presents research results, test methods, and performance criteria related to the structural and environmental functions and the durability and safety characteristics of building elements and systems.

Technical Notes-Studies or reports which are complete in themselves but restrictive in their treatment of a subject. Analogous to monographs but not so comprehensive in scope or definitive in treatment of the subject area. Often serve as a vehicle for final reports of work performed at NIST under the sponsorship of other government agencies.

Voluntary Product Standards-Developed under procedures published by the Department of Commerce in Part 10, Title 15, of the Code of Federal Regulations. The standards establish nationally recognized requirements for products, and provide all concerned interests with a basis for common understanding of the characteristics of the products. NIST administers this program in support of the efforts of private-sector standardizing organizations.

Consumer Information Series-Practical information, based on NIST research and experience, covering areas of interest to the consumer. Easily understandable language and illustrations provide useful background knowledge for shopping in today's technological marketplace.

Order the above NIST publications from: Superintendent of Documents, Government Printing Office, Washington, DC 20402.

Order the following NIST publications-FIPS and NISTIRs-from the National Technical Information Service, Springfield, VA 22161.

Federal Information Processing Standards Publications (FIPS PUB)-Publications in this series collectively constitute the Federal Information Processing Standards Register. The Register serves as the official source of information in the Federal Government regarding standards issued by NIST pursuant to the Federal Property and Administrative Services Act of 1949 as amended, Public Law 89-306 (79 Stat. 1127), and as implemented by Executive Order 11717 (38 FR 12315, dated May 11, 1973) and Part 6 of Title 15 CFR (Code of Federal Regulations).

NIST Interagency Reports (NISTIR)-A special series of interim or final reports on work performed by NIST for outside sponsors (both government and non-government). In general, initial distribution is handled by the sponsor; public distribution is by the National Technical Information Service, Springfield, VA 22161, in paper copy or microfiche form. 
U.S. Department of Commerce

National Institute of Standards and Technology 325 Broadway

Boulder, Colorado 80305-3337

\section{Official Business}

Penalty for Private Use, $\$ 300$ 